\title{
Chordality in Matroids: In Search of The Converse to Hliněný's Theorem
}

\author{
By \\ Andrew Martin Probert \\ Supervisor: Professor Geoff Whittle
}

\section{VICTORIA UNIVERSITY OF WELLINGTON \\ Te Whare Wānanga o te Ūpoko o te Ika a Māui}

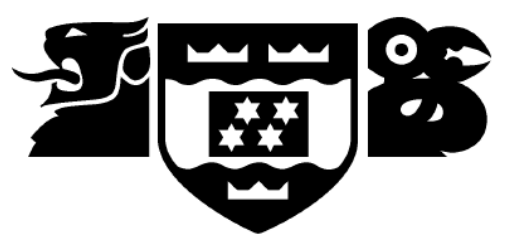

School of Mathematics and Statistics

Te Kura Mātai Tatauranga, Rangahau Pūnaha

\author{
A thesis \\ submitted to the Victoria University of Wellington \\ in fulfilment of the requirements for the degree of \\ Doctor of Philosophy \\ in Mathematics.
}

Victoria University of Wellington

2018 



\begin{abstract}
Bodlaender et al. [7] proved a converse to Courcelle's Theorem for graphs [15] for the class of chordal graphs of bounded treewidth. Hliněný [25] generalised Courcelle's Theorem for graphs to classes of matroids represented over finite fields and of bounded branchwidth. This thesis has investigated the possibility of obtaining a generalisation of chordality to matroids that would enable us to prove a converse of Hliněný's Theorem [25].
\end{abstract}

There is a variety of equivalent characterisations for chordality in graphs. We have investigated the relationship between their generalisations to matroids. We prove that they are equivalent for binary matroids but typically inequivalent for more general classes of matroids.

Supersolvability is a well studied property of matroids and, indeed, a graphic matroid is supersolvable if and only if its underlying graph is chordal. This is among the stronger ways of generalising chordality to matroids. However, to obtain the structural results that we need we require a stronger property that we call supersolvably saturated.

Chordal graphs are well known to induce canonical tree decompositions. We show that supersolvably saturated matroids have the same property. These tree decompositions of supersolvably saturated matroids can be processed by a finite state automaton. However, they can not be completely described in monadic second-order logic.

In order to express the matroids and their tree decompositions in monadic second-order logic we need to extend the logic over an extension field for 
each matroid represented over a finite field. We then use the fact that each maximal round modular flat of the tree decomposition for every matroid represented over a finite field, and in the specified class, spans a point in the vector space over the extension field. This enables us to derive a partial converse to Hliněný's Theorem. 


\section{Acknowledgements}

I would like to thank all those who have helped and supported me during my 3 years. First of all, I am inordinately grateful to my supervisor Professor Geoff Whittle for such close and structured support. I would not have survived without it. I would also like to thank Professor Rod Downey my secondary supervisor for his useful suggestions and support and in particular for proposing the problem that we try to solve in this thesis.

Much thanks go also to the mathematics team at Vic - in particular Dillon for his feedback and useful ideas. I would like to thank the crazy graduates of the school of mathematics and statistics. In particular Room 429 (Susan, Will and Meenu) and Room 464 (Del, Sebastian and Jessica) and Nick. Thanks again also to the administrative staff at the school and to Steven Archer of course. Thanks Michael for your useful template.

I would like to thank my Mum and extended family for their ongoing and unquestioning support.

A final shout out goes to Sascha and the crew of the Vic pharmacy! Thanks for allowing me to spend hours hiding in the pharmacy when I needed to escape. 



\section{Contents}

1 Introduction 3

1.1 The Structure of this Thesis . . . . . . . . . . . . . . . . 5

\begin{tabular}{lll}
\hline 2 & Preliminaries & 7
\end{tabular}

2.1 Graphs . . . . . . . . . . . . . . . . . 7

2.2 Matroids . . . . . . . . . . . . . . . . . . . . . . . . . . . . . . . 9

2.3 Represented Matroids . . . . . . . . . . . . . . . . . . . . . . . . . 10

2.4 Extension Fields . . . . . . . . . . . . . . . . . . . . . . 11

2.5 Tree and Branch Decompositions . . . . . . . . . . . . . . . . 12

$2.5 .1 \quad$ Branch Decompositions of Matroids . . . . . . . . . . 15

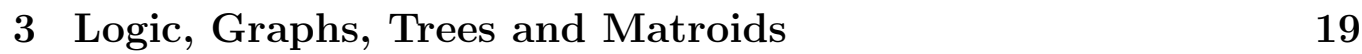

3.1 Words . . . . . . . . . . . . . . . . . . . . . 20

3.1.1 Recognisability for Words . . . . . . . . . . . . 25

3.1 .2 Regularity and Myhill-Nerode . . . . . . . . . . 26

3.1 .3 Büchi's Theorem . . . . . . . . . . . . . . . . . . 27

3.2 Graphs . . . . . . . . . . . . . . . . . . . . 28 


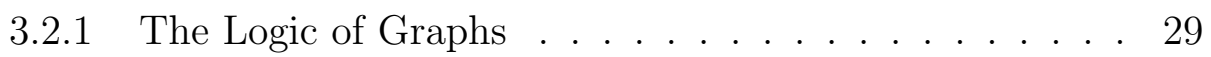

3.2 .2 Treewidth . . . . . . . . . . . . . . . . 31

3.2.3 Recognising the Tree Decomposition of a Graph . . . . 32

3.2 .4 Courcelle's Theorem [15] . . . . . . . . . . . . . . . . . 32

3.3 Trees . . . . . . . . . . . . . . . . . . . . . . . . . . 33

3.3.1 Courcelle's Theorem for Trees [15] . . . . . . . . . . . 35

3.4 Matroids . . . . . . . . . . . . . . . . . . . 36

$3.4 .1 \quad$ Branch Decompositions . . . . . . . . . . . . . 36

$3.4 .2 \quad$ A Monadic Second-Order Logic for Matroids . . . . . . 36

3.4 .3 Tree Automata . . . . . . . . . . . . . . . 37

$3.4 .4 \quad$ A Myhill-Nerode Analogue . . . . . . . . . . . . . . . 38

$3.4 .5 \quad$ Hliněný's Theorem [25] $\ldots$. . . . . . . . . . . . . . . . . . . 39

3.5 Courcelle's Conjecture . . . . . . . . . . . . . . . . . . . 40

3.5.1 Trying to Prove the Conjecture . . . . . . . . . . . . 41

3.5.2 Bodlaender, Heggernes and Telle . . . . . . . . . . 41

$3.5 .3 \quad$ A Converse for Matroids . . . . . . . . . . . . . . . . 43

$\begin{array}{|ll|}4 & \text { Modularity and Roundness }\end{array}$

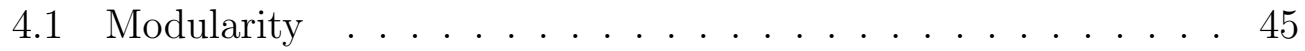

4.2 Roundness . . . . . . . . . . . . . . . . . . . . . . . . . . . . . . . . 57

\begin{tabular}{|lll}
5 & Chordality in Graphs & 63
\end{tabular}

5.1 Chordal Graphs . . . . . . . . . . . . . . . . . . . . 63 


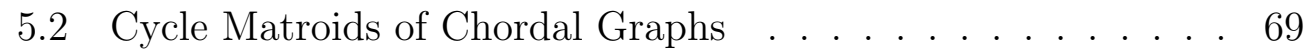

\begin{tabular}{lll}
\hline 6 & Chordality in Matroids & 73
\end{tabular}

$6.1 \quad$ Some Background . . . . . . . . . . . . . . . . . 73

$6.1 .1 \quad$ Alternative Definitions of Chordality . . . . . . . . 73

6.1 .2 The History of These Definitions . . . . . . . . . . 75

6.2 Chordality for Matroids in General . . . . . . . . . . . . 76

6.3 Chordality in Binary Matroids . . . . . . . . . . . . . . . . 79

6.4 Further Discussion . . . . . . . . . . . . . . . . . . . . . . 82

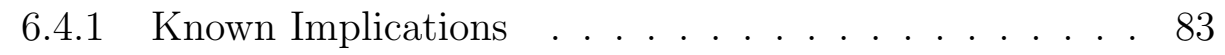

6.4 .2 Known Non-implications . . . . . . . . . . . . 84

$6.4 .3 \quad$ Open Problems . . . . . . . . . . . . . . . . . . . . 89

\begin{tabular}{lll}
\hline 7 & Supersolvability & 93
\end{tabular}

7.1 Supersolvablity in Matroids . . . . . . . . . . . . . . . 93

7.1.1 Supersolvability in the Cycle Matroids of Graphs . . . 95

7.1 .2 Supersolvability for Binary Matroids . . . . . . . . . 96

7.1.3 Supersolvability and Chordality for General Matroids . 98

7.2 Supersolvable Saturation . . . . . . . . . . . . . . . . . . . . . 99

$7.3 \quad$ Bridges and Minimal Separations . . . . . . . . . . . . . 106

7.4 Modular Separations . . . . . . . . . . . . . . . . 111

7.4.1 Some Consequences of Modular Separations . . . . . . 114 
$\begin{array}{lll}8 & \text { Tree Decompositions and Matroids } & 119\end{array}$

8.1 Matroidal Tree Decompositions . . . . . . . . . . . . . . . . . 119

8.2 Supersolvably Saturated Matroids . . . . . . . . . . . . . . . 121

$9 \quad$ A Partial Converse to Hliněný 133

9.1 Establishing a Theorem . . . . . . . . . . . . . . . . 133

9.2 Colouring Supersolvable Matroids . . . . . . . . . . . . . 136

9.3 Definability of Tree Decompositions . . . . . . . . . . . . . 139

9.4 Using an Extension Field . . . . . . . . . . . . . . . . . . . . . 142

\begin{tabular}{ll}
\hline 10 Conclusions & 149
\end{tabular}

10.1 Open Questions . . . . . . . . . . . . . . . . 153 


\section{List of Figures}

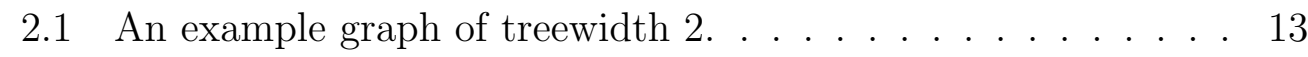

$2.2 \quad$ A possible tree decomposition of width 2. . . . . . . . . . . 13

2.3 The bags hierarchy or tree decomposition for above tree decomposition ..................... 13

2.4 An example graph of branchwidth $2 . \quad \ldots \ldots \ldots$

2.5 A possible branch decomposition of width 2. The edge labels represent the "guts" of the $k$-separation created by removing an edge from the tree. . . . . . . . . . . . . 16

$4.1 \quad M\left(K_{4}\right)$ - An example where $\mathrm{cl}_{M}(F \cup G)$ is not modular . . . . 57

6.1 How the different implications of chordality in matroids relate. The arrows indicate the direction of implication. The edge labels refer to the Lemmas that establish the implications. . . 83

6.2 How the different non-implications of chordality in matroids relate. $A-\mathrm{X} B$ means " $A$ does not imply $B$ ". The edge labels indicate counter-examples for each case. . . . . . . . . . 85

$6.3 \quad U_{3,5}$ - the uniform matroid of 5 points on the plane . . . . . 85

$6.4 \quad L_{2,3}$ - the rank 3 matroid with two lines one with 3 points and one with 2 points . . . . . . . . . . . . . 87 
6.5 The rank 4 matroid with 8 elements. This is of course the rank-4 whirl. . . . . . . . . . . . . . . . . . . . . . 88

6.6 How the different open problems relate. $A-? \quad B$ means that we do not know if $A$ implies $B$. The edge labels refer to the open problems that describe each case. . . . . . . . . . . . 89

$7.1 \quad B_{3,10}:$ A counter-example to supersolvable matroids being strongly chordal . . . . . . . . . . . . . . . . . . . . . . 99

7.2 A rank 4 matroid that shows that supersolvability does not imply saturation . . . . . . . . . . . . 100

7.3 A rank 6, 18 element, supersolvably saturated matroid $M_{6,18} \cdot 115$

8.1 A rank 6, 18 element, supersolvably saturated matroid $M_{6,18} \cdot 130$

$8.2 \quad$ A rotunda tree for $M_{6,18} \ldots \ldots \ldots \ldots$

$9.1 \quad$ Fano plane $F_{7}$ or $P G(2,2)$ with extension line $\ldots . . . . .144$ 


\section{Chapter 1}

\section{Introduction}

In this thesis we will investigate matroid structure. In particular, we will focus on chordality and related structure for general matroids. We are motivated by a long-standing problem in computability and logic.

In 1991 Courcelle [15] proved a theorem showing that for general graphs of bounded treewidth if a property of a graph is definable in monadic secondorder logic then it is recognisable by an automaton. In the same paper Courcelle conjectured that the converse was true. In the 26 years since, there have been a number of attempts to partially prove this conjecture including an important result by Bodlaender, Heggenes and Telle [7]. In 2016 Bojanczyk and Pilipczuk [8] provided a full proof for the conjecture. We are interested in proving a partial analogue of Courcelle's conjecture for matroids represented over a finite field.

Hliněný [25] has proved a theorem generalizing the result of Courcelle [15]. We have been motivated in this thesis by this problem of finding a partial converse to Hliněný's theorem. This will involve identifying structure in some class of matroids that we can leverage to allow an automaton to run over the matroids. To paraphrase Courcelle [16] it is not at all clear how an automaton should traverse a matroid since a general matroid has no obvious structure. A word or a tree is essentially its own algebraic structure [16]. 
The usual technique for graphs is to find a way to generate a tree decomposition from the graph. We shall try to follow the same process for matroids. The question is when do matroids give us a nice tree decomposition?

The main part of this thesis is an investigation of structure in matroids that might allow us to construct a tree decomposition for any such structured matroid. We start looking at matroid structure from modular flats to roundness to saturation (where every round flat is modular). We observe that chordal graphs have a nice tree of cliques. This leads us into an investigation of chordality in matroids.

We look at a number of alternative definitions of chordality for matroids. We carefully choose five definitions of chordality in matroids ranging from the very weak to the very strong. We observe that for binary and for graphic matroids these definitions are equivalent but for general matroids they differ - sometimes to a significant degree.

There is a result originally given by Dirac [18] and Stanley [47] that shows that supersolvability in the cycle matroids of graphs is equivalent to graph chordality. As a result we will turn our attention to supersolvability. This is more promising because it involves a matroid having a chain of modular flats. Unfortunately, this is not enough. In any tree decomposition we construct we want the intersection of the bags to be round and modular. Supersolvability does not guarantee that every round flat is modular. As a result, we must explicitly require that our matroids be supersolvable and saturated.

We see that supersolvable saturation is exactly the sort of structure we need. The only issues left include the important requirement that our separations be well behaved. However, as we have shown, in supersolvable saturated matroids any minimal vertical separation is what we call a modular separation and is well-behaved. We can capture the concept of the guts as a round 
modular flat whose rank will be exactly the connectivity of the separation.

This then allows us to construct a well-behaved tree decomposition for any supersolvably saturated matroid. As we will see every supersolvably saturated matroid has a graph of round modular flats which we call rotundas. We can apply an algorithm to find a maximum weighted spanning tree of the graph. Any such tree is an optimal tree decomposition for the matroid. It is a matroidal tree decomposition in the language of Hliněný and Whittle [27] such that its treewidth is exactly the rank of the largest rotunda in the tree. Furthermore, this maximum rank is bounded from above and below by the branchwidth of the matroid.

Given this we will turn to the original problem that motivated this thesis. First, we are interested in a partial converse to Hliněný's Theorem. This means we are required to only consider supersolvably saturated matroids of bounded branchwidth that are represented over a finite field. We look at how to take the tree decomposition we have shown exists for such matroids and show that we can describe the process of an automaton running over it in counting monadic second-order logic. As we will see there are a number of issues we must overcome. We describe each issue and our approach to addressing it. We hope we may have paved the way for future investigations of this problem.

\subsection{The Structure of this Thesis}

A quick guide to this thesis is as follows. Here I will indicate where a chapter includes mostly previously known results or includes original material.

\section{- Chapter 2}

We begin by introducing some preliminary concepts 


\section{- Chapter 3}

We give the historical background to the problem that will occupy this thesis.

\section{- Chapter 4}

In this chapter we give, as background, some well-known material about modularity and roundness in matroids that will be used in later chapters.

\section{- Chapter 5}

This chapter gives a background to chordal graphs and consists entirely of known results.

\section{- Chapter 6}

We discuss in detail when and how chordality as a concept can be extended to matroids. Most of this material is original.

\section{- Chapter 7}

We introduce supersolvability in matroids and in particular supersolvably saturated matroids as well as discussing when separations are nicely behaved. Apart from the first few subsections most of this chapter includes original material.

\section{- Chapter 8}

We establish a new result that supersolvably saturated matroids have canonical tree decompositions.

\section{- Chapter 9}

Finally, we discuss to what extent we can use the tools and concepts acquired in the previous chapters to prove a partial converse to Hliněný's Theorem. 


\section{Chapter 2}

\section{Preliminaries}

In this preliminary chapter we give some introductory definitions for key concepts used elsewhere in the thesis. We introduce graphs and matroids at least in the terms we will need later. We also define representation of matroids, extension fields and tree and branch decompositions.

\subsection{Graphs}

Let $V$ be a finite non-empty set of vertices and let $E$ be a multiset of 2element multisubsets of vertices called edges then $G=(V, E)$ is a graph. If every edge has exactly two vertices and there is never more than one edge with the same pair of vertices (i.e. no parallel edges) then $G$ is a simple graph. We shall assume for the purpose of this thesis that all graphs are simple unless otherwise stated.

Let $G=(V, E)$. Let $H=\left(V^{\prime}, E^{\prime}\right)$ be a subgraph of $G$ if the vertices of $H$ form a subset of $V$ (that is $V^{\prime} \subseteq V$ ) and the edges $E^{\prime}$ of $H$ form a subset of those edges in $E$ whose vertices are in $V^{\prime}$. A path is a sequence of distinct vertices, such that for each vertex in the sequence, except for the final vertex, there is an edge containing it and the next vertex in the sequence. Two vertices are 
adjacent if there exists an edge between them. The neighbours of a vertex $v$ are all those vertices which are adjacent to $v$. We denote the neighbours of vertex $v$ in the graph $G$ as $\mathcal{N}_{G}(v)$.

A connected graph is a graph for which for any two vertices there exists a path between them. A cycle is a sequence of vertices where the length of the sequence is at least two and the start and end vertices are adjacent to each other. An acyclic graph is a graph for which there exist no cycles.

A tree is a connected acyclic graph. A subtree is a subgraph of a tree. A rooted tree $(G, z)$ is a tree comprised of graph $G=(V, E)$ with a root $z$ chosen from the set of vertices $V$. A free tree or an unrooted tree is a tree which has no root vertex. A vertex $v$ is the parent of $u$, if $u$ is a child of $v$.

The complete graph on $n$ vertices is a graph in which there is an edge between every pair of the $n$ vertices. We denote this graph by $K_{n}$. A planar graph is a graph which can be embedded in the plane so that no edges cross. An isomorphism of two graphs $G$ and $H$ is generally described as a bijection $f: V(G) \rightarrow V(H)$ (that is between the vertex sets of $G$ and $H$ ) such that any two vertices in $G, u$ and $v$, are only adjacent if and only if $f(u)$ is adjacent to $f(v)$ in $H$.

A contraction of the edges of a graph $G$ is an operation whereby an edge is removed while simultaneously the two vertices previously joined are merged into one. For two graphs $G$ and $H$, the graph $H$ is a minor of the graph $G$ if a graph isomorphic to $H$ can be obtained from $G$ by taking a subgraph and then deleting and/or contracting (possibly zero) edges. 


\subsection{Matroids}

Refer to Oxley [41] and Hliněný [25] for the following. A matroid can be defined in a number of different ways. We will give two such definitions. First, a matroid is a pair $M=(E, \mathcal{B})$ where $E(M)$ is the ground set of $M$ and $\mathcal{B} \subseteq 2^{E(M)}$ is a collection of bases of $M$. These satisfy the following rules:

B1. $\mathcal{B}$ is nonempty.

B2. If $B_{1}$ and $B_{2}$ are members of $\mathcal{B}$ and $x \in B_{1}-B_{2}$, then there is an element $y \in B_{2}-B_{1}$ such that $\left(B_{1}-x\right) \cup y \in \mathcal{B}$.

Alternatively, a matroid is defined if it has a rank function, $r_{M}: 2^{E(M)} \rightarrow$ $\mathbb{N} \cup\{0\}$, that satisfies the following rules:

R1. If $X \subseteq E(M)$, then $0 \leq r_{M}(X) \leq|X|$.

R2. If $X \subseteq Y$, then $r_{M}(X) \leq r_{M}(Y)$.

R3. (submodularity) If $X$ and $Y$ are subsets of $E(M)$, then $r_{M}(X \cup Y)+$ $r_{M}(X \cap Y) \leq r_{M}(X)+r_{M}(Y)$.

The rank $r_{M}(F)$ for $F \subseteq E(M)$ is the cardinality of a basis of $M \mid F$. For $r_{M}(M)$ we write $r(M)$.

The cycle matroid of a graph $G$ is a matroid on the groundset $E(G)$ and is denoted $M(G)$. The bases of $M(G)$ are the spanning forests of $G$ and the circuits, minimal dependent sets, are the cycles of $G$. Then $r_{M(G)}(F)=$ $|V(G[F])|-\omega(G[F])$ where $F \subseteq E(G)$ and $G[F]$ is the subgraph induced by $F$ and $\omega(G[F])$ is the number of components of $G[F]$. Another example of a type of matroid is the column matroid where $E(M)$ is a finite set of column vectors. Such matroids have the usual linear dependency, bases, rank etc. 
The connectivity function $\lambda_{M}: 2^{E(M)} \rightarrow \mathbb{N} \cup\{0\}$ is defined for all subsets $A \subseteq E(M)$ by $\lambda_{M}(A)=r_{M}(A)+r_{M}(E(M)-A)-r(M)$. We can also write $\lambda_{M}(A, E(M)-A)$. A subset $A \subseteq E(M)$ is $k$-separating if $\lambda_{M}(A) \leq k-1$. A partition $(A, E(M)-A)$ is called a $k$-separation if $A$ is $k$-separating and both $|A|,|E(M)-A| \geq k$. A $k$-separation is vertical if $\lambda_{M}(A) \leq k-1$ and $\min \left\{r_{M}(A), r_{M}(E(M)-A)\right\} \geq k-1$. An alternative definition is that a $k$-separation is vertical if $\operatorname{cl}_{M}(A) \nsupseteq E(M)-A$ and $\operatorname{cl}_{M}(E(M)-A) \nsupseteq A$. Finally, for $n>1$, a matroid is called $n$-connected if it has no $k$-separation for $k=1,2, \ldots, n-1$. We say that a matroid is connected if it is 2 -connected.

\subsection{Represented Matroids}

A field $\mathbb{F}$ is a structure satisfying axioms with respect to operations called addition and multiplication. The real numbers $\mathbb{R}$ for example form a field. Fields can also be finite provided that their cardinality is a prime power. These are referred to as Galois Fields, and are denoted $G F(q)$, where $q=p^{k}$ for some prime number $p$ and some positive integer $k$. See Oxley [41]. We can represent matroids over fields including finite fields (see below). In doing so we closely follow the definitions given in Hliněný [25].

Let $A$ be a matrix and let $M[A]$ be the matroid whose elements $E(M)$ are the column vectors of $A$. Let the independent subsets of $E(M)$ be the linearly independent sets of column vectors of $A$. Then $M[A]$ is the matroid represented by $A$.

Let $P G(n, \mathbb{F})$ be the projective geometry over $\mathbb{F}^{n+1}$. Let $X \subseteq P G(n, \mathbb{F})$. Then $\langle X\rangle$ is the span of $X$.

A basis of a projective space is a maximal sized independent subset of the vector space. Again let $X \subseteq P G(n, \mathbb{F})$. Then the rank of $X, r(X)$, is the cardinality of the basis contained by $X$. A projective transformation between 
two projective spaces is a linear transformation between the underlying vector spaces.

A representation $M[A]$ is loopless if it contains no zero elements. A loopless representation $M[A]$ is a multiset of points in the projective space $P G(n, \mathbb{F})$ where $n=r(E(M[A]))$.

A point configuration is a finite multiset of points in a projective space of $\mathbb{F}$. Two point configurations in projective spaces over $\mathbb{F}$ are projectively equivalent if there is a single projective transformation that maps between them. An $\mathbb{F}$-represented matroid is a projective equivalence class of point configurations over $\mathbb{F}$.

To obtain an actual instance of an $\mathbb{F}$-represented matroid we just choose an arbitrary point configuration from the equivalence class. Note that several inequivalent representations over a field may exist for the same matroid.

\subsection{Extension Fields}

An extension field is a field which contains another defined field [37]. We can sometimes describe the extension field as an algebraic statement over elements of the contained field. Given the above section any $\mathbb{F}$-represented matroid being essentially a particular point configuration over some projective space can be "contained" in a larger projective space over an extension field $\mathbb{W}$ such that $\mathbb{F} \subseteq \mathbb{W}$. That is $P G(n, \mathbb{F}) \subseteq P G(n, \mathbb{W})$ where $n=[\mathbb{W}: \mathbb{F}]$. In fact, the degree of the extension can be any number.

If $\mathbb{F}$ is the finite field $G F(q)$ and $\mathbb{W}$ is finite with dimension $n$ over $\mathbb{F}$ then $\mathbb{W}$ has $q^{n}$ elements. We denote $\mathbb{W}$ in this case as $G F\left(q^{n}\right)$. 


\subsection{Tree and Branch Decompositions}

Robertson and Seymour [42] introduced the concept of bounded width decompositions of graphs in the early 1980s in their proof of the Graph Minors Theorem. Their focus was primarily on the use of such structures in finding obstruction sets with respect to graphs of minor quasi-order. However, these path/tree decomposition structures have proved useful leading to efficient algorithms for bounded parameter values for many natural input restrictions of $N P$-complete problems. In general many graph problems can be solved in linear time when the input also includes a bounded width decomposition of the graph.

The origins of branchwidth and treewidth are closely connected to the proof of the Graph Minors Theorem. This theorem states that in an infinite list of graphs there exist two graphs $H$ and $G$ such that $H$ is a minor of $G$. Tree and branch decompositions have since proved important because they allow graphs to decomposed into more manageable pieces in such a way that we know how to reconstruct the graph.

Branchwidth has some advantages over treewidth as a fixed parameter. While finding optimal branchwidth is NP-hard for general graphs as is finding the optimal treewidth [5] it has been proven that one can find the branchwidth and indeed a branch decomposition for a planar graph in polynomial time [45]. No such equivalent algorithm exists for treewidth. Moreover, when rooted, branch decompositions always form binary trees giving a much simpler structure.

Formally we have the following [42].

A tree decomposition of a graph $\mathrm{G}$ is a pair $(T, \beta)$ where $T$ is a tree and $\beta: V(T) \rightarrow 2^{V(G)}$ is a function mapping nodes of $T$ to subsets of vertices of $G$, which we call bags. A tree decomposition must satisfy the following 


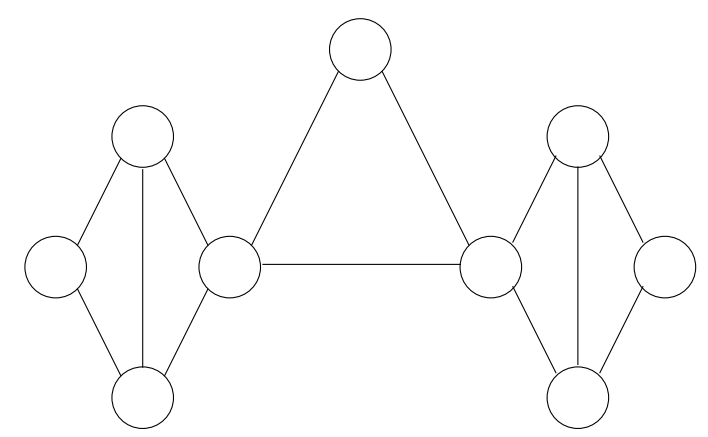

Figure 2.1: An example graph of treewidth 2.

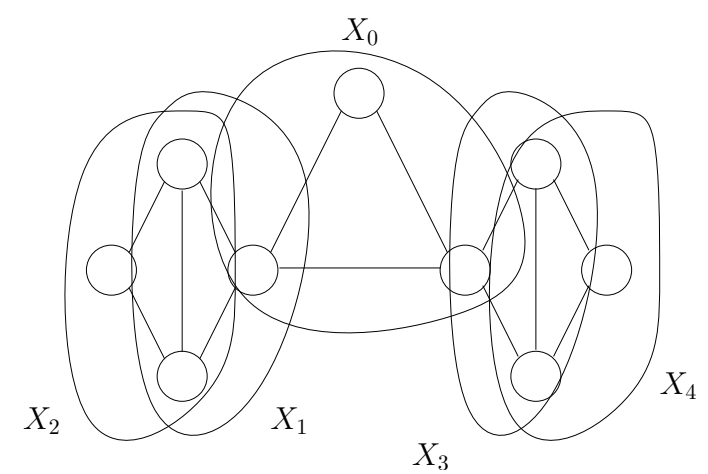

Figure 2.2: A possible tree decomposition of width 2 .

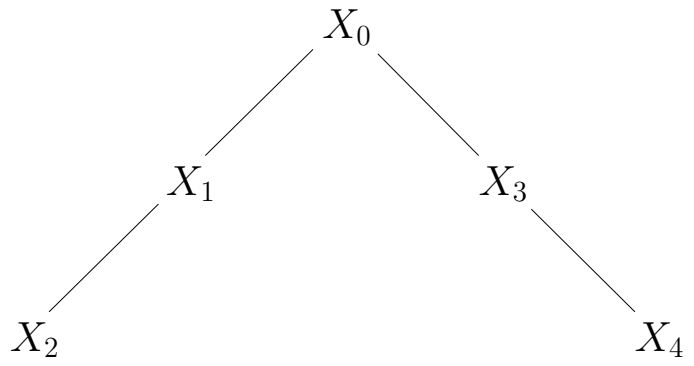

Figure 2.3: The bags hierarchy or tree decomposition for above tree decomposition 
properties.

1. The union of all the $\beta(t)$ for $t$ in $V(T)$ is $V(G)$

2. For every edge $e$ of $G$ there exists $t$ in $V(T)$ such that $e$ has both ends in $\beta(t)$

3. For every $t, t^{\prime}, t^{\prime \prime}$ in $V(T)$ if $t^{\prime}$ is on the path of $T$ between $t$ and $t^{\prime \prime}$ then $\beta(t) \cap \beta\left(t^{\prime \prime}\right) \subseteq \beta\left(t^{\prime}\right)$. This is known as interpolation.

The treewidth of the tree decomposition is the maximum, across all $t$, of $|\beta(t)|-1$. We subtract 1 to ensure that actual trees have treewidth 1 . The graph $G$ has treewidth $w$ if $w$ is the minimum such that $G$ has a tree decomposition of width $w$.

Given a graph $G=(V, E)$ a branch decomposition is a pair $(T, \mu)$ where $T$ is a tree with every internal node ternary and $\mu$ is a bijection $\mu: E \rightarrow l(T)$ from $E$ to the leaves of $T$ (denoted by $l(T)$ ).

If we remove any edge of $T$ we partition $E$ into two sets $A$ and $E-A$. Then the width of this edge is exactly the number of vertices in common between $A$ and $E-A$ or connectivity of $(A, E(M)-A)$. In turn the maximum such width for a given decomposition tells us how interconnected the decomposition is. We call this measure the width of the decomposition.

We can then ask what decomposition out of all the possible decompositions of a graph has the smallest width. We call this smallest width the branchwidth and any decomposition whose width is equal to this branchwidth is called optimal. Thus branchwidth can be seen as a measure of the best way of decomposing a given graph $G$ - it tells us what is the smallest width for any decomposition. 


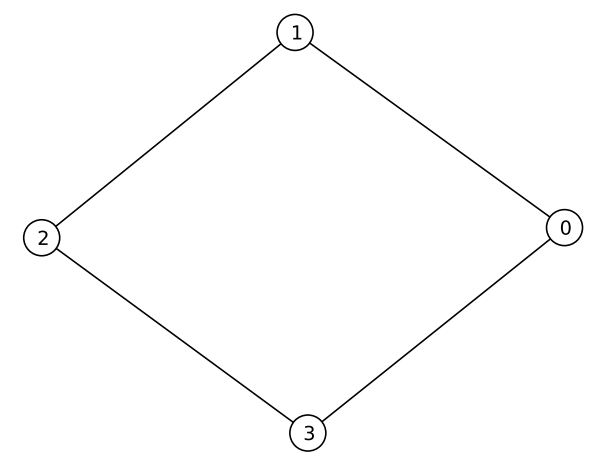

Figure 2.4: An example graph of branchwidth 2.

Treewidth and branchwidth for graphs are closely related as are tree decompositions and branch decompositions. Robertson and Seymour [43] have shown that the branchwidth $b w(G)$ and treewidth $t w(G)$ of a graph $G$ are related by

$$
b w(G) \leq t w(G)+1 \leq \frac{3}{2} b w(G)
$$

\subsubsection{Branch Decompositions of Matroids}

Unlike graphs, as we have seen, matroids do not have vertices but only elements of the groundset. These are similar to the edges in graphs. As a result we can not talk about a set of vertices in common to two parts of the matroid. However, we can talk about separations and the size of the connectivity of these separations. We define a branch decomposition for a matroid $M$ as follows.

Given $M$ let $T$ be a tree where every internal node is ternary. Let $l(T)$ denote the leaves of $T$. Then a branch decomposition of $M$ is a pair $(T, \mu)$ where $\mu$ 


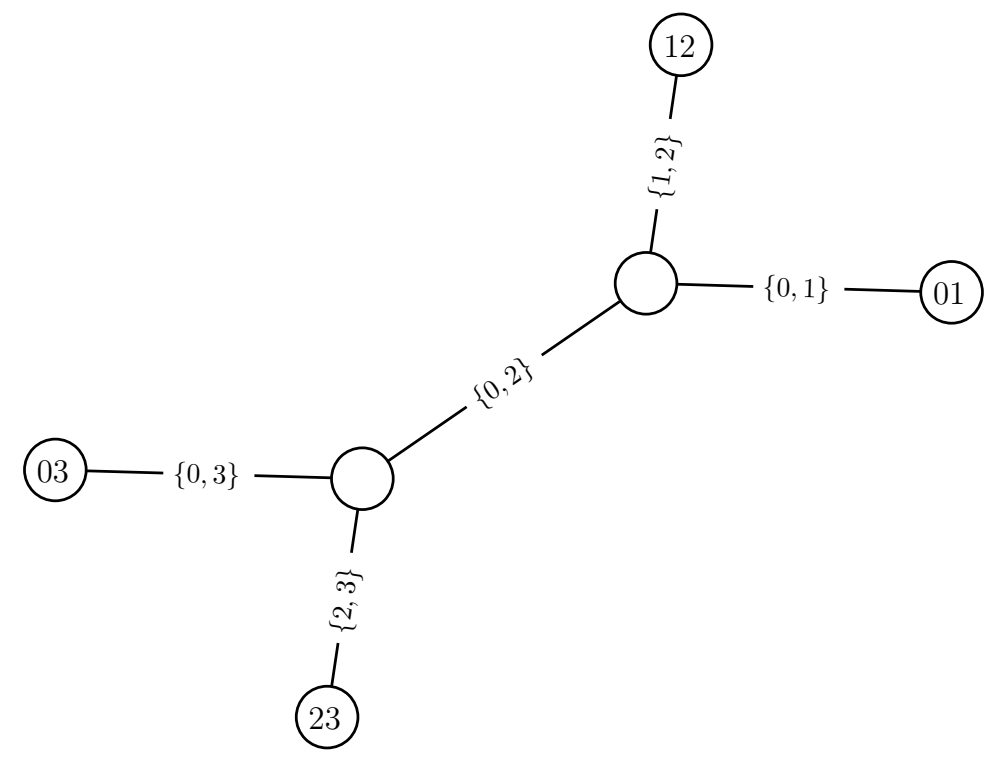

Figure 2.5: A possible branch decomposition of width 2. The edge labels represent the "guts" of the $k$-separation created by removing an edge from the tree. 
is a bijection $\mu: E(M) \rightarrow l(T)$ from $E(M)$ to the leaves of $T$. If we remove any edge of $T$ we partition $E(M)$ into two sets $A$ and $E(M)-A$. Then the width of this edge is exactly the connectivity of $(A, E(M)-A)$ or $\lambda(A)$.

In turn the maximum such width for a given decomposition tells us how interconnected the decomposition is. We call this measure the width of the decomposition. We can then ask what decomposition out of all the possible decompositions of a graph has the smallest width. We call this smallest width the branchwidth and any decomposition whose width is equal to this branchwidth is called optimal. 


\section{Chapter 3}

\section{Logic, Graphs, Trees and Matroids}

We now introduce the core problem that motivates this thesis. As we will see this problem is a fairly long-standing one. In short, suppose there is a collection of mathematical objects. Now assume there is a logic or "theory" that describes the space occupied by these objects. Then we ask two questions.

First, suppose there is a sentence that describes some subset of these objects. Then we ask given such a theorem is it possible to use a computer to determine if any object belongs to this subset. Second, suppose we can computably "recognise" that an object belongs to some subset. Then we ask given such computable recognition of the subset must there be a theorem which describes membership of the subset.

We discuss this problem from the point of view of words, graphs, trees and matroids. The first question has been positively answered for all four classes of object when we have bounded branchwidth (or treewidth). For each we discuss the nature of the object involved, how to formulate logical statements over the domain of such objects, and how to computably recognise classes of such objects. In each case we state a theorem which ties these concepts 
together.

Our main interest is in the second question. In the process we discuss Courcelle's Conjecture [16] the analogue of which we are trying to prove. Observe that the formulation of recognisability and of the logic varies across classes of objects since we are following the language of the key authors in each case.

\section{$3.1 \quad$ Words}

In 1960 Büchi [13] published a seminal paper in which among other results he showed that a language is recognisable if and only if it can be described by a sentence in Monadic Second Order Logic. We shall begin by discussing Büchi's result.

A domain is the set of possible values a variable in a logic can take. We begin with the domain of words. A letter is a symbol and an alphabet $\Sigma$ is a set of letters. A word or string $w_{n}$ of length $n \in \mathbb{N}$ is an ordered sequence of letters in some alphabet $\Sigma$. Moreover, $\Sigma^{*}$ is the set of all possible words given the alphabet $\Sigma$. There is a map from the sequence $[1, \ldots, n]$ to $\Sigma$. We call each element of $1, \ldots, n$ a position in $w_{n}$ and each position has an associated letter.

For an alphabet $\Sigma$ and a class of words over $\Sigma$ we define the Monadic SecondOrder Logic for Words which we abbreviate as $M S$ in the language of Mayhew, Newman and Whittle [39]. The syntax of $M S$ is as follows [39]. Given a word $w_{n}$ the domain of $M S$ is defined to be the set of positions in $w_{n}$ namely $\{1,2, \ldots, n\}$. We include the following symbols. We include variables $x_{1}, \ldots, x_{n}$ for individual positions in the word. We further include $X_{1}, \ldots, X_{m}$ for sets of positions. We also include the constants $\emptyset, 0,1,2, \ldots$.

We include the function symbols $\{\cdot\}, \cdot^{-}, \cup$ and $\cap$. We have the relation 
symbols $=, \in, \subseteq$ as well as the logic symbols $\neg, \vee, \wedge, \exists, \forall$. Observe that $<$ is interpreted as is usual in number theory. The quantifiers $\forall, \exists$ are applicable to both positions and sets of positions and the relation $=$ represents equality for both positions and their sets. We include the following predicates:

1. $Q_{a}(x)$, which is true if the letter at position $x$ is $a$.

2. $\operatorname{Succ}(x, y)$, which is true if $y$ is the position which follows $x$.

3. $\operatorname{first}(x)$, which is true if $x$ is the first position in $w$.

4. last $(x)$, which is true if $x$ is the last position in $w$.

Following Mayhew, Newman and Whittle [39] we recursively define formulas in $M S$ and at the same time define their sets of variables. The predicates we listed above as well as the relations $x=y, x \in X, X \subseteq Y$ and $x<y$ are called atomic formulas. A non-atomic formula, or formula for short, is an expression generated by a finite application of the following rules. Every formula $\psi$ has an associated set of variables $\operatorname{Var}(\psi)=\operatorname{var}_{x}(\psi) \cup \operatorname{var}_{X}(\psi)$ and free variables $\operatorname{Free}(\psi)=f r_{x}(\psi) \cup f r_{X}(\psi)$ where $\operatorname{var}_{x}(\psi)$ and $f r_{x}(\psi)$ relate to individual variables and $\operatorname{var}_{X}(\psi)$ and $f r_{X}(\psi)$ relate to set variables:

1) Every atomic formula, $\psi$, is a formula, and $\operatorname{Free}(\psi)=\operatorname{Var}(\psi)$.

2) If $\psi$ is a formula, then $\neg \psi$ is a formula, and $\operatorname{Var}(\neg \psi)=\operatorname{Var}(\psi)$ while $\operatorname{Free}(\neg \psi)=\operatorname{Free}(\psi)$.

$3)$ If $\psi_{1}$ and $\psi_{2}$ are formulas, and $\operatorname{Free}\left(\psi_{i}\right) \cap\left(\operatorname{Var}\left(\psi_{j}\right)-\operatorname{Free}\left(\psi_{j}\right)\right)=\emptyset$ for $\{i, j\}=\{1,2\}$, then $\psi_{1} \wedge \psi_{2}$ is a formula, and $\operatorname{Var}\left(\psi_{1} \wedge \psi_{2}\right)=$ $\operatorname{Var}\left(\psi_{1}\right) \cup \operatorname{Var}\left(\psi_{2}\right)$, while $\operatorname{Free}\left(\psi_{1} \wedge \psi_{2}\right)=\operatorname{Free}\left(\psi_{1}\right) \cup \operatorname{Free}\left(\psi_{2}\right)$. 
4) If $\psi$ is a formula and $X_{i} \in \operatorname{Free}(\psi)$, then $\exists X_{i} \psi$ is a formula, and $\operatorname{Var}\left(\exists X_{i} \psi\right)=\operatorname{Var}(\psi)$, while $\operatorname{Free}\left(\exists X_{i} \psi\right)=\operatorname{Free}(\psi)-\left\{X_{i}\right\}$.

A variable in $\operatorname{Var}(\psi)$ is free if it is in $\operatorname{Free}(\psi)$, and bound otherwise [39]. A formula is quantifier-free if all of its variables are free, and is a sentence if all of its variables are bound. If $\psi$ is a quantifier-free formula, then we define the depth of $\psi$ to be the number of applications of Rules 2), 3) and 4) above required to construct $\psi$. Rule 3) insists that no variable can be free in one of $\psi_{1}$ and $\psi_{2}$ and bound in the other, if $\psi_{1} \wedge \psi_{2}$ is to be a formula. We can overcome this constraint if necessary by renaming the bound variables in a formula.

Note that if $\psi$ is a formula and $X_{i} \in \operatorname{Free}(\psi)$, then we use $\forall X_{i} \psi$ as a shorthand for $\neg\left(\exists X_{i} \neg \psi\right)$. We also use the shorthand $\psi_{1} \vee \psi_{2}$ to mean $\neg\left(\left(\neg \psi_{1}\right) \wedge\left(\neg \psi_{2}\right)\right)$ and we use $\psi_{1} \rightarrow \psi_{2}$ to mean $\left(\neg \psi_{1}\right) \vee \psi_{2}$. Likewise, we use $\psi_{1} \longleftrightarrow \psi_{2}$ to mean $\left(\psi_{1} \rightarrow \psi_{2}\right) \wedge\left(\psi_{2} \rightarrow \psi_{1}\right)$. We use $X \nsubseteq Y$ to stand for $\neg(X \subseteq Y)$.

Now let $\Sigma$ be some alphabet and let $W \subseteq \Sigma^{*}$ be some class of words. Let $p_{w}^{a}$, for $w \in W$ and $a \in \Sigma$, be the set of positions of letter $a$ in $w$. Then let $P_{w}$ be the set of positions in $w \in W$. That is $P_{w}$ is defined for some particular word $w \in W$. Then let $\psi$ be a formula in $M S$. We define an interpretation of $\psi$ to be a triple $\left(P_{w}, \tau_{x}, \tau_{X}\right)$ where $\tau_{x}$ is a function from $f r_{x}(\psi)$ into $P_{w}$ and $\tau_{X}$ is a function from $f r_{X}(\psi)$ into the power set of $P_{w}$. We recursively define what it means for $\left(P_{w}, \tau_{x}, \tau_{X}\right)$ to satisfy $\psi$. We begin by assuming that $\psi$ is atomic. Then we have these rules:

- If $\psi$ is $X_{i} \subseteq X_{j}$ for $X_{i}, X_{j} \in \operatorname{var}_{X}(\psi)$, then $\left(P_{w}, \tau_{x}, \tau_{X}\right)$ satisfies $\psi$ if and only if $\tau_{X}\left(X_{i}\right) \subseteq \tau_{X}\left(X_{j}\right)$.

- If $\psi$ is $x_{i}=x_{j}$ for $x_{i}, x_{j} \in \operatorname{var}_{x}(\psi)$, then $\left(P_{w}, \tau_{x}, \tau_{X}\right)$ satisfies $\psi$ if and 
only if $\tau_{x}\left(x_{i}\right)=\tau_{x}\left(x_{j}\right)$.

- If $\psi$ is $x_{i}<x_{j}$ for $x_{i}, x_{j} \in \operatorname{var}_{x}(\psi)$, then $\left(P_{w}, \tau_{x}, \tau_{X}\right)$ satisfies $\psi$ if and only if $\tau_{x}\left(x_{i}\right)<\tau_{x}\left(x_{j}\right)$.

- If $\psi$ is $x_{i} \in X_{i}$ for $x_{i} \in \operatorname{var}_{x}(\psi)$ and $X_{i} \in \operatorname{var}_{X}(\psi)$, then $\left(P_{w}, \tau_{x}, \tau_{X}\right)$ satisfies $\psi$ if and only if $\tau_{x}\left(x_{i}\right) \in \tau_{X}\left(X_{i}\right)$.

- If $\psi$ is $Q_{a}\left(x_{i}\right)$ for $x_{i} \in \operatorname{var}_{x}(\psi)$ and $a \in w$ for some $w \in W$, then $\left(P_{w}, \tau_{x}, \tau_{X}\right)$ satisfies $\psi$ if and only if $\tau_{x}\left(x_{i}\right) \in p_{w}^{a}$.

- If $\psi$ is $\operatorname{Succ}\left(x_{i}, x_{j}\right)$ for $x_{i}, x_{j} \in P_{w}$, then $\left(P_{w}, \tau_{x}, \tau_{X}\right)$ satisfies $\psi$ if and only if $\tau_{x}\left(x_{j}\right)-\tau_{x}\left(x_{i}\right)=1$.

- If $\psi$ is $\operatorname{first}\left(x_{i}\right)$ for $x_{i} \in P_{w}$, then $\left(P_{w}, \tau_{x}, \tau_{X}\right)$ satisfies $\psi$ if and only if $\tau_{x}\left(x_{i}\right)=1$.

- Finally, if $\psi$ is $\operatorname{last}\left(x_{i}\right)$ for $x_{i} \in P_{w}$, then $\left(P_{w}, \tau_{x}, \tau_{X}\right)$ satisfies $\psi$ if and only if for some $w \in W$ it follows that $\tau_{x}\left(x_{i}\right)=|w|$.

Next suppose that $\psi$ is not atomic. Then we have the rules:

- If $\psi$ is $\neg \phi$ for some formula $\phi$, then $\left(P_{w}, \tau_{x}, \tau_{X}\right)$ satisfies $\psi$ if and only if $\left(P_{w}, \tau_{x}, \tau_{X}\right)$ does not satisfy $\phi$.

- If $\psi$ is $\phi_{1} \wedge \phi_{2}$, then $\left(P_{w}, \tau_{x}, \tau_{X}\right)$ satisfies $\psi$ if and only if $\left(P_{w}, \tau_{x} \uparrow_{f r_{x}\left(\phi_{1}\right)}\right.$ , $\left.\tau_{X} \uparrow_{f r_{X}\left(\phi_{1}\right)}\right)$ satisfies $\phi_{1}$ and $\left(P_{w}, \tau_{x}\left\lceil_{f r_{x}\left(\phi_{2}\right)}, \tau_{X} \uparrow_{f r_{X}\left(\phi_{2}\right)}\right)\right.$ satisfies $\phi_{2}$. 
- If $\psi$ is $\exists x_{i} \phi$ for $x_{i} \in f r_{x}(\phi)$, then $\left(P_{w}, \tau_{x}, \tau_{X}\right)$ satisfies $\psi$ if and only if there is an element $y \in P_{w}$ such that the interpretation $\left(P_{w}, \tau_{x} \cup\right.$ $\left.\left\{\left(x_{i}, y\right)\right\}, \tau_{X}\right)$ satisfies $\phi$.

- If $\psi$ is $\exists X_{i} \phi$ for $X_{i} \in f r_{X}(\phi)$, then $\left(P_{w}, \tau_{x}, \tau_{X}\right)$ satisfies $\psi$ if and only if there is a subset $Y \subseteq P_{w}$, such that the interpretation $\left(P_{w}, \tau_{x}, \tau_{X} \cup\right.$ $\left.\left\{\left(X_{i}, Y\right)\right\}\right)$ satisfies $\phi$.

Note that if $\psi$ is an $M S$ sentence, then we can say that $W$ satisfies $\psi$ (or $\psi$ is satisfied by $W)$ if the interpretation $\left(P_{w}, \tau_{x}, \tau_{X}\right)$ satisfies $\psi$. Alternatively we can say that $P_{w}$ and so $W$ is $M S$-definable.

Example 3.1.1. In $M S$ we can express ideas such as "the word contains the sequence of letters "aba" with the statement $\exists x, y, z Q_{a}(x) \wedge(\operatorname{Succ}(x, y) \wedge$ $\left.Q_{b}(y)\right) \wedge\left(\operatorname{Succ}(y, z) \wedge Q_{a}(z)\right)$.

We extend $M S$ to include a counting predicate. Counting monadic secondorder logic for words, abbreviated to $C M S$, is defined by Courcelle [15] as the extension of $M S$ by the unary predicate symbols $\bmod _{p, q}$ where $p<q$ are non-negative integers. This has the intended meaning: $\bmod _{p, q}(\mathbf{V})=$ True iff $|X|=p \bmod q$, where $X$ is a set of positions and $V$ is the variable for the set $X$. Sometimes we use the predicate symbol $\operatorname{card}_{p, q}(X)$ to mean $|X| \bmod q=p$. Observe that in this case we must also add the symbols $||,$. and $<$.

Example 3.1.2. Consider a word $w$ over $\Sigma=\{a, b\}$. Then suppose we assert that there is some sequence of letters in $w$ such that this sequence has an even number of letters e.g. xaby or xaaaay or xbbaabby where $\mathrm{x}$ and $y$ are other parts of the word. We can define this with the statement $\left.\exists X\left[\forall x \in X \exists y \in X(\operatorname{Succ}(x, y) \vee \operatorname{Succ}(y, x)) \wedge \operatorname{card}_{0,2}(X)\right]\right)$ 


\subsubsection{Recognisability for Words}

We want to test that a given word belongs to the class of words we are interested in. To achieve this we use a mechanism called a Finite Automaton.

A deterministic finite automaton is a quintuple $M=\left(Q, \Sigma, q_{0}, F, \delta\right)$ where

- $Q$ is a finite set called the set of states,

- $\Sigma$ is a finite set of symbols, termed the alphabet, and

- $q_{0} \in Q$ is called the initial state,

- $F \subseteq Q$ are the accepting states,

- $\delta: Q \times \Sigma \rightarrow Q$ is a function called the transition function.

As seen in Downey [20] we will usually denote states by $\left\{q_{i}: i \in G\right\}$ for some set $\mathrm{G}$. We can think of automata as machines that act to accept or reject strings from $\Sigma^{*}$ and we consider the machine $M$ as starting on the leftmost symbol of a string $\sigma \in \Sigma^{*}$, in state $q_{0}$ then moving from state to state as it processes each symbol.

A non-deterministic finite automaton (or $N F A$ ) is a quintuple $M=(Q, \Sigma, S, F, \delta)$ where

- $Q$ is a finite set called the set of states,

- $\Sigma$ is a finite set of symbols, termed the alphabet, and 
- $S \subseteq Q$ are the initial states,

- $F \subseteq Q$ are the accepting states,

- $\delta: Q \times \Sigma \rightarrow \mathcal{P}(Q)$ is the non-deterministic transition function.

We can see that a $N F A$ is structurally similar to a $D F A$ except that it can transition to multiple states at once and can have multiple starting states. It has been shown (see Sipser [46]) that DFAs and NFAs are equivalent that is they accept exactly the same languages.

A language over words over some alphabet $\Sigma$ is recognisable if it is possible to construct a deterministic finite automaton which accepts or rejects a given word as belonging to the language. By the Myhill-Nerode theorem, given next, this in turn implies that it is possible to "process" the language, using a finite automaton, in finite time.

\subsubsection{Regularity and Myhill-Nerode}

We call a relation $R$ on a set $\Sigma^{*}$ a right congruence (with respect to concatenation) if and only if

(a) $R$ is an equivalence relation on $\Sigma^{*}$;

(b) for all $x, y \in \Sigma^{*}, x R y$ iff for all $z \in \Sigma^{*}, x z R y z$. 
For a language $L$, the canonical right congruence (induced by $L$ ) is the relation $\sim_{L}$ defined by

$x \sim_{L} y$ iff for all $z \in \Sigma^{*}, x z \in L$ iff $y z \in L$.

Note that "an equivalence relation on $\Sigma^{*}$ is said to have finite index if it has only a finite number of equivalence classes over $\Sigma^{*} "[20$. L is finite state if it can be recognised by a finite state automaton.

Theorem 3.1.3 (The Myhill-Nerode theorem [20]).

1. The following are equivalent over $\Sigma^{*}$ :

(a) L is finite state;

(b) $L$ is the union of a collection of equivalence classes of a right congruence of finite index over $\Sigma^{*}$;

(c) $\sim_{L}$ has finite index.

2. Furthermore, any right congruence satisfying (b) is a refinement of $\sim_{L}$.

This can be restated to say that a language $L$ is regular (recognisable by some automaton) if and only if it is finite index.

\subsubsection{Büchi's Theorem}

We now turn to a significant result which ties together the Monadic SecondOrder Logic of Words $(M S)$ and recognisability by a finite automaton. This 
is the well-known Büchi's Theorem which is the historical inspiration for the work in this thesis.

Given an alphabet $\Sigma$ and some set of words $W$ over $\Sigma$ (e.g. all words containing the sequence "aba") how does the logical power of describing the class in $M S$ compare with the power of recognizing the set of words using a finite automaton? Büchi proved that the power of logically defining such a class is equal to that of recognizing the class.

Theorem 3.1.4 (Büchi (1960) [13]). A language is definable in second-order monadic logic if and only if it is recognisable.

In the standard proof usually given for this theorem one first shows that recognisability of words implies definability in $M S$. It is shown that one can construct a logical statement that describes the transition function of an automaton such that if the automaton accepts a word then the logical statement is true. This operates over the logic for words.

Next it is shown that definability in $M S$ implies recognisability by a finite state automaton. To achieve this it is usually shown that one can construct an automaton with a transition function that models a special logical statement that accepts a word if, as the transition function traverses the statement, it is ever true (and rejects if the statement is ever false).

\subsection{Graphs}

We now investigate how this problem might relate to more complex objects such as graphs. We follow Courcelle [15] in doing this. We will see that we can not do what we did for words for graphs in general. We need further structure and the usual approach is to require a tree decomposition. 
A finite alphabet $\Sigma$ is ranked if there is a function $\alpha: \Sigma \rightarrow \mathbb{N}$. If $a \in \Sigma$ then $\alpha(a)$ is called the arity of $a$. Let $A$ be a ranked alphabet with a mapping $\tau: A \mapsto \mathbb{N}$ so that for $a \in A$ we see that $\tau(a)$ is the type of $a$.

Consider general graphs as defined earlier. Now extend a graph to include a boundary on which graphs can be glued together. Then let $F G(A)_{k}$ be the family of graphs labeled by elements of $A$ with a boundary size of $k$. Then $F G(A)$ is all graphs for any $k$.

\subsubsection{The Logic of Graphs}

A graph $G \in F G(A)$ is a logical structure with two domains consisting of vertices and edges. Let $A$ be a ranked alphabet. The symbols in this logic include $\mathbf{v}$ for vertices, $\mathbf{e}$ for edges, $s_{i}$, called a source, for a constant vertex for every $1 \leq i \leq k$ and $e d g_{a}$ which is a predicate symbol for every $a \in A$. That is the graph is edge-labeled. With $G \in F G(A)_{k}$ for some $k$ we associate the logical structure $<V_{G}, E_{G},\left(s_{i G}\right)_{i \in[k]},\left(e d g_{a G}\right)_{a \in A}>$ where $V_{G}$ is the domain of vertices, $E_{G}$ is the domain of edges, $s_{i G}$ is the $i$ th source of $G$ and $e d g_{a G}\left(e, v_{1}, v_{2}\right)$ is true if and only if $e$ has label $a$ and the vertices of $e$ are $v_{1}, v_{2}$. (In fact Courcelle [15] does this for hypergraphs).

We build formulas in this logic by using variables $u, x, y, \ldots$ and set variables $X, Y, \ldots$ of vertex or edge kind. These are always finite since $F G(A)$ is finite. Let $W$ be a sorted set of variables $\left\{u, u^{\prime}, \ldots, U, U^{\prime}, \ldots\right\}$ each of them being vertices or edges. Then we can let the set $W_{s}=W \cup\left\{s_{1}, \ldots, s_{k}\right\}$. Then we can define the atomic formulas $\mathcal{A}_{A, k, q}(W)$ as

- $u=u^{\prime}$ for $u, u^{\prime} \in W_{s}$ where $u$ and $u^{\prime}$ are of the same kind.

- $u \in U$ for $u, U \in W_{s}$ where $U$ is a set of elements of the same kind as $u$. 
- $e d g_{a}\left(u, u_{1}^{\prime}, u_{2}^{\prime}\right)$ where $u, u_{1}^{\prime}, u_{2}^{\prime} \in W_{s}$ and $u$ is an edge variable and $u_{1}^{\prime}, u_{2}^{\prime}$ are vertex variables.

- $\operatorname{card}_{n, p}(U)$ where $U \in W_{s}, 0 \leq n<p, 2 \leq p<q$.

Remark 3.2.1. $\operatorname{card}_{n, p}(X)=$ true if and only if $|X|=n \bmod p$.

We now define $\mathcal{C} \mathcal{L}_{A, k, q}^{(h)}(W)$ to be the set of formulas defined inductively as

i) $\phi \in \mathcal{C L}_{A, k, q}^{(h)}(W)$ if $\phi \in \mathcal{A}_{A, k, q}(W)$

ii) $\phi_{1} \wedge \phi_{2}, \phi_{1} \vee \phi_{2}, \neg \phi_{1} \in \mathcal{C} \mathcal{L}_{A, k, q}^{(h)}(W)$ if $\phi_{1}, \phi_{2} \in \mathcal{C L}_{A, k, q}^{(h)}(W)$

iii) $\exists u \phi, \forall u \phi \in \mathcal{C} \mathcal{L}_{A, k, q}^{(h)}(W)$ if $\phi \in \mathcal{C} \mathcal{L}_{A, k, q}^{(h)}(W \cup\{u\}), u \notin W$

iv) $\exists U \phi, \forall U \phi \in \mathcal{C} \mathcal{L}_{A, k, q}^{(h)}(W)$ if $\phi \in \mathcal{C} \mathcal{L}_{A, k, q}^{(h)}(W \cup\{U\}), U \notin W$

The parameter $h$ denotes the depth of nested quantifications for the formula $\phi$. Then the least $h$ such that $\phi \in \mathcal{C L}_{A, k, q}^{(h)}(W)$ is called the height of $\phi$.

Notation 3.2.2. We let $\mathcal{C} \mathcal{L}_{A, k, q}(W):=\bigcup\left\{\mathcal{C L}_{A, k, q}^{(h)}(W) \mid h \geq 0\right\}$ and $\mathcal{C L}_{A, k}(W):=$ $\bigcup\left\{\mathcal{C} \mathcal{L}_{A, k, q}(W) \mid q \geq 2\right\}$. Often we drop the subscripts $A, k$ and $q$.

Finally, a property of graphs in $F G(A)_{k}$ for some type $k$ is $\mathcal{C} \mathcal{L}$-definable if there exists a closed formula $\phi \in \mathcal{C} \mathcal{L}_{A, k}$ such that $G$ satisfies this property if and only if $\phi$ holds in $G$. A set $L \subseteq F G(A)_{k}$ is $\mathcal{C} \mathcal{L}$-definable if the membership in $L$ is such. For shorthand we can refer to a property as definable and mean that it is $\mathcal{C} \mathcal{L}$-definable. 


\subsubsection{Treewidth}

As mentioned a natural question arises as to whether we can prove a result like Büchi's Theorem for graphs in general. There is however the obvious problem that while one can clearly run an automaton over a path or word it is not so clear how one can run an automaton over a general form graph. The usual solution to this problem is to require some extra structure in the graph in particular a tree-like structure. We shall first describe two ways such tree-like structure is defined and show their equivalence. We shall then give a theorem of Courcelle [15] that shows that, as long as a class of graphs has bounded treewidth, if it is definable in a Counting Monadic Second-Order Logic such as that defined earlier, then it must be recognisable by an automaton.

We saw in Chapter 2 the definition of tree decomposition given by Robertson and Seymour [42]. For the following we shall be talking about simple graphs. Let $G$ be such a graph. A $k$-clique in a graph $G$ is a subset $K \subseteq V(G)$ of size $k$ such that there is an edge between every two vertices in $K$. A graph $G$ is a $k$-tree if either

- $G$ is the complete graph on $k$ vertices; or

- $G$ is obtained recursively from a $k$-tree $G^{\prime}$ by attaching a new vertex $v$ to a $k$-clique $K$ in $G^{\prime}$ such that $v$ is a neighbour of $k$ vertices in $K$.

A partial $k$-tree is any subgraph of a $k$-tree.

Theorem 3.2.3 (Robertson and Seymour [42]). The set of partial $k$-trees is equivalent to the set of graphs with treewidth at most $k$.

The preceeding theorem is useful as inspiration for our later work with tree decompositions of certain classes of matroid. 


\subsubsection{Recognising the Tree Decomposition of a Graph}

Courcelle [15] defines a generic concept of automaton to be a triple $(h, A, C)$ where $A$ is an algebraic structure, $C$ is a set of final states and $h$ is a homomorphism that maps a recognisable set to $A$. Where an automatic structure is needed Courcelle further defines transition functions based upon this generic automaton.

We can model this automaton using a standard leaf to root tree automaton $\left(Q, A, \delta=\delta_{v} \cup \delta_{e}, q_{0}, F\right)$. $Q$ is the finite set of states. $\delta$ is the transition function made up of a transition mapping for vertices, $\delta_{v}$, and a transition mapping for edges $\delta_{e}$, which both map states to states. $q_{0}$ is the initial state at the leaves and $F$ is the set of possible accepting states. If $r$ is the root vertex we accept if $\delta_{v}(r) \in F$.

\subsubsection{Courcelle's Theorem [15]}

Remember that $F G(A)_{k}$ is the set of all graphs over a ranked alphabet $A$ with $k$ distinguished vertices forming its boundary. Even if we can not decide that a set $X$ is finite, if it is given as a finite list of elements, then we say it is effectively given [15].

Theorem 3.2.4 (Courcelle [15]). Every definable subset of $F G(A)_{k}$ is an effectively given recognisable set of graphs.

Essentially, in proving this theorem, Courcelle [15] shows that for any class of graphs with bounded treewidth $k$ it is possible to recognise members of that class if it is possible to describe the class using the logic $\mathcal{C} \mathcal{L}$. This theorem has also been stated so that the requirement for bounded treewidth is explicit as follows. 
Theorem 3.2.5 (Courcelle's Theorem as given by Downey and Fellows [20]). If $F$ is a family of graphs described by a sentence $\phi$ in second-order monadic logic with a counting predicate, then the relation $\sim_{F}$ has finite index in the large universe of t-boundaried graphs.

In proving these theorems it is shown that for each input statement $\phi$ and set of bounded $t$-treewidth graphs we can test whether the input graph can support the statement (that is "partially equipped" in the language of Downey and Fellows [20]). Courcelle shows this inductively on the length of $\phi$ by examining all the possible different forms the statement $\phi$ can take.

The theorem has been extended by Arnborg et al [2]. The corollary [2] that one can recognise a graph of bounded treewidth in polynomial time if it can be represented in MSO logic has proved quite important.

\subsection{Trees}

Now we consider logic and recognisability for trees and in particular for languages involving labeling of trees. Courcelle has shown that for unordered unranked edge-labeled trees recognisability by a finite automaton is equivalent to definability by a sentence in a counting monadic second-order logic.

Again we follow the language of Courcelle [15]. Let $A$ be a finite ranked alphabet consisting of symbols of type 1 or 2 where the type indicates the number of vertices in the associated edge. Then we can let $A_{i}, i \in\{1,2\}$, be the subsets of $A$ for each type. Given this a tree is a graph $G$ in $F G(A)_{1}$ satisfying the following. Observe that the trees have a bound of size $k=1$. We call the singular source for each tree the root.

1. For every vertex $v \in V_{G}$ there is a path from the root of the tree to $v$. 
2. Every vertex different from the root is the target of one and only one edge of type 2 .

3. Every edge is labeled with an element from $A$.

4. The root is the target of no edge.

If an edge is of type 2 then we call it binary. We have the tree operations $|$. and $\hat{. .}|$.$| joins two existing trees together and \hat{.}$ joins a vertex to a tree. In brief with both of these operations we first take the disjoint sum then "fuse" the roots of each together and finally redefine so that the resulting tree has only one root node. $\|$ is both associating and commutative and we treat it as an infix operation. Observe that if $G$ and $G^{\prime}$ are trees then so are $G \| G^{\prime}$ and $\hat{b}(G)$ (where $\hat{b}(G)$ means the operation of joining a vertex $b$ to the tree $G$ - Courcelle [15] uses this notation). We let $T(A)$ be our class of trees just as $F G(A)$ is our class of graphs. In fact $T(A)$ is algebraic over $F G(A)$ since

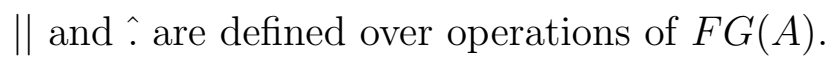

The logic for trees is exactly the logic for graphs given earlier but applied only to the objects in $T(A)$ and using its operations. Observe that a subset $L \subseteq T(A)$ is $\mathcal{C} \mathcal{L}$-definable if and only if it is definable as a set of trees since $T(A)$ is $\mathcal{C} \mathcal{L}$-definable as a subset of $F G(A)_{1}$.

Furthermore, as with graphs Courcelle [15] defines a generic concept of automaton which we can model using a standard leaf to root tree automaton $\left(Q, A, \delta=\delta_{v} \cup \delta_{e}, q_{0}, F\right) . \quad Q$ is the finite set of states. $\delta$ is the transition function made up of a transition mapping for vertices, $\delta_{v}$, and a transition mapping for edges, $\delta_{e} . q_{0}$ is the initial state at the leaves and $F$ is the set of possible accepting states. If $r$ is the root vertex we accept if $\delta_{v}(r) \in F$. 


\subsubsection{Courcelle's Theorem for Trees [15]}

The actual theorem is as follows. Observe that the sets $\operatorname{Rec}(F G(A)) \subseteq$ $F G(A)$ and $\operatorname{Rec}(T(A)) \subseteq T(A)$ are those sets recognisable by an automaton.

Theorem 3.3.1 (Courcelle [15]). Let $L \subseteq T(A)$. Then the following are equivalent:

i) $L \in \boldsymbol{\operatorname { R e c }}(F G(A))$.

ii) $L \in \boldsymbol{R e c}(T(A))$.

iii) $L$ is $\mathcal{C} \mathcal{L}$-definable.

Our prime interest in this thesis is in statement ii) implying iii). Courcelle himself states that he proves the result that statement iii) implies i) in his theorem for graphs given earlier. He proves that ii) implies iii) by showing that he can construct transition functions mapping movement through a labeled unbounded and unordered tree and describe them using a sentence in $\mathcal{C L}$.

Courcelle describes separate transition functions for the automaton for the vertices of the input tree as opposed to the edges of the tree. These functions test against the edge labels at the current position the automaton is with respect to the tree. This is in essence similar to the standard proof given for Büchi's Theorem for the same direction. 


\subsection{Matroids}

Next, we consider how the problem under discussion can be extended to matroids.

Hliněný [25] established the analogue for Courcelle's Theorem for matroids that are represented over a finite field. This is a very special subclass of matroids that includes all graphs as well as vector subspaces. Hliněný has generalized Courcelle's theorem showing that for any class of represented matroids over a fixed field with bounded branchwidth it is possible to recognise members of that class if it is possible to describe the class using a counting monadic second-order logic.

\subsubsection{Branch Decompositions}

First a note about branch decompositions. See Chapter 2 for definitions of branchwidth and branch decomposition for graphs and matroids. We adopt the notation $b w(M)$ and $b w(G)$ for the branchwidth of matroid $M$ and graph $G$. As we saw for graphs the branchwidth and treewidth of a graph bound each other so can be treated as equivalent for these purposes.

\subsubsection{A Monadic Second-Order Logic for Matroids}

Next we must define a monadic second order logic for matroids. The syntax for the counting monadic second-order logic for matroids represented over a finite field, abbreviated to $C M S_{M}$ by Hliněný [25], which is defined over the class of matroids, represented over a finite field, includes the following symbols. We include variables $x_{1}, \ldots, x_{n}$ for individual elements in the represented space that is vectors in the vector space. We further include $X_{1}, \ldots, X_{m}$ for element sets. We also include the constants $\emptyset, 0,1,2, \ldots$ as well as $E$. 
We further include the function symbols $|\cdot|,\{\cdot\}, \cdot^{-},+, \cup$ and $\cap$. In addition we have the relation symbols $=, \in, \subseteq$ as well as the logic symbols $\neg, \vee, \wedge, \exists, \forall$. The quantifiers $\forall, \exists$ are applicable to both element and set variables and the relation $=$ represents equality for both elements and their sets. In addition we include the following predicates (or formulas):

1. indep $(F)$, where $F$ is a set variable, and the predicate is true iff $F$ is an independent set in the matroid.

2. $\operatorname{card}_{p, q}(X)$ means $|X| \bmod q=p$.

3. we write $\phi \rightarrow \psi$ to stand for $\neg \phi \vee \psi$.

4. in addition we add the following specific predicates for matroids derived from those above
i. $X \supseteq Y \equiv \forall x(x \in X \vee x \notin Y)$
ii. $X \subseteq Y \equiv \forall x(x \in Y \vee x \notin X)$
iii. $X \subset Y \equiv X \subseteq Y \wedge X \neq Y$
iv. $\neg X \equiv \forall x(x \in E-X)$

\subsubsection{Tree Automata}

We now give an alternative formulation of a tree automaton to that used earlier by Courcelle [15]. A rooted ordered sub-binary tree is such that each of its vertices has at most 2 children ordered left to right. Let $\Sigma$ be a finite 
alphabet of symbols. Then $\Sigma^{* *}$ is the class of rooted ordered sub-binary trees with vertices labeled from $\Sigma$.

A leaf to root tree automaton $\left(K, \Sigma, \partial_{t}, q_{0}, F\right)$ has a set of states $K$, an alphabet $\Sigma$, a transition function $\partial_{t}: K \times K \times \Sigma \rightarrow K$, a starting state $q_{0}$ and a set of accepting states $F$. Let $\mathcal{A}$ be a leaf to root tree automaton. Let $T \in \Sigma^{* *}$ be a rooted ordered sub-binary tree. Let $T_{L}$ and $T_{R}$ be the left and right subtrees of $T$ or $\emptyset$ if they do not exist. Let $a$ be the symbol from $\Sigma$ assigned to the root node of $T$. Then we can in turn define a function $t-e v a l_{\mathcal{A}}: \Sigma^{* *} \rightarrow K$ recursively as follows:

- $t-\operatorname{eval}_{\mathcal{A}}(\emptyset)=q_{0}$

- $t-e v a l_{\mathcal{A}}(T)=\partial_{t}\left(t-\operatorname{eval}_{\mathcal{A}}\left(T_{L}\right), t-\operatorname{eval}_{\mathcal{A}}\left(T_{R}\right), a\right)$

Then $T$ is accepted by $\mathcal{A}$ if $t$-eval $(T) \in F$. A tree language $\mathcal{L} \subseteq \Sigma^{* *}$ is called finite state if it is accepted by some tree automaton.

\subsubsection{A Myhill-Nerode Analogue}

Hliněný [25] defines the concept a of fixed size $t$-boundaried matroid which is similar to Courcelle's concept of a boundaried graph and of parse tree which is similar to a labeled tree decomposition and over which a tree automaton would run. Parse trees have fixed width boundaries as well. He gives the following result.

Lemma 3.4.1. An $\mathbb{F}$-represented matroid $M$ has branchwidth at most $t+1$ if and only if $M$ is parsed by a spanning t-boundaried parse tree. 
Next he gives an analogue to the Myhill-Nerode theorem for graphs. Let $\mathcal{B}_{t}$ be the set of all $\mathbb{F}$-represented matroids that have branchwidth at most $t$. Let $T_{t} \subset \Pi_{t-1}^{* *}$ be the language of all $(t-1)$-boundaried parse trees over $\mathbb{F}$ with the (ranked) alphabet $\Pi_{t-1}$. Let $\overline{\mathcal{B}}_{t}$ be the set of all $(t-1)$-boundaried matroids parsed by trees from $T_{t}$.

Then suppose that $\mathcal{M}$ is a set of represented matroids. We say $\mathcal{M}$ is finite state if the collection of all parse trees parsing the members of $\mathcal{M}$ is MyhillNerode finite state. We say that $\mathcal{M}$ is $t$-width finite state for $t \geq 1$ if the restriction $\mathcal{M} \cap \mathcal{B}_{t}$ is finite state.

Theorem 3.4.2 (Myhill-Nerode Analogue for Represented Matroids). Let $t \geq 1$ and let $\mathbb{F}$ be a finite field. A set of $\mathbb{F}$-represented matroids $\mathcal{M}$ is $t$-width finite state if and only if the equivalence $\approx_{\mathcal{M}, t}$ has finite index over $\overline{\mathcal{B}}_{t}$.

\subsubsection{Hliněný's Theorem [25]}

Before stating his theorem, Hliněný [25] gives an updated formulation of Courcelle's theorem [15] similar to that restated by Downey and Fellows [20] given earlier. $M S_{2}$ is a counting monadic second-order logic for graphs similar to the logic $\mathcal{C L}$ of Courcelle [15].

Theorem 3.4.3 (Courcelle's Theorem). If $\mathcal{G}$ is a family of graphs described by a sentence in $M S_{2}$ for graphs then $\mathcal{G}$ is $t$-width finite state for every $t \geq 1$.

He then gives the following theorem.

Theorem 3.4.4. Let $\mathbb{F}$ be a finite field. If $\mathcal{M}$ is a set of $\mathbb{F}$-represented matroids described by a sentence in the logic $C M S_{M}$ over matroids then $\mathcal{M}$ is $t$-width finite state for every $t \geq 1$. 
Hliněný [25] proves this result by induction on the length of the statement $\phi$. Essentially he proves the inductive statement given below.

Lemma 3.4.5. Let $\phi$ be a formula in the $C M S_{M}$ logic of matroids and let $\sigma$ be a partial equipment signature for $\phi$. Then $\approx_{\phi}$ has finite index on the universe of $\sigma$-partially equipped $u$-boundaried matroids.

Observe that a logical formula, or statement, is partially equipped if the free variables of the formula, given the domain of the logic, support the formula (see [20]). A partial equipment signature $\sigma$ is a collection of free variables that one uses to support a formula [20]. A formula is $\sigma$-partially equipped if there is a partial equipment signature $\sigma$ that supports the formula [20].

Hliněný proves the induction by first showing that the relation $\approx_{\phi}$ is finite index for each of the atomic formulas for $C M S_{M}$. He then shows that for each of the operations based on the connectors $\wedge, \neg, \exists$ the relation $\approx_{\phi}$ based on the statement $\phi$ must also be finite index. As he points out we can obtain $\checkmark$ and $\forall$ from these connectors.

\subsection{Courcelle's Conjecture}

As we have seen Büchi's Theorem (Theorem 3.1.4) and Courcelle's Theorem for Trees (Theorem 3.3.1) are proofs in both directions. That is recognisability is shown to equal definability for words and for unbounded unordered trees. However, Courcelle's Theorem for graphs (Theorem 3.2.4 and Hliněný's Theorem (Theorem 3.4.4) are in one direction only whereby definability implies recognisability but not the converse. Soon after giving his famous theorem for graphs (Theorem 3.2.4) Courcelle conjectured [16] that the converse is true but again only for graphs of bounded treewidth. 


\subsubsection{Trying to Prove the Conjecture}

Since this conjecture was made a number of graph theorists have been working to prove the result though for restricted cases. Courcelle himself proved the conjecture for graphs of bounded treewidth $k$ where $k \leq 2$ (ie for seriesparallel graphs) [16]. Kaller [31] and Bodlaender and Jaffke [28] have showed that the conjecture in fact holds for graphs of treewidth 3 (e.g. Halin graphs) and also for $k$-connected graphs. Bodlaender et al [7] have shown that the conjecture holds if we also require our graphs to be chordal or of bounded chordality.

As of late 2016 Bojanczyk and Pilipczuk [8] have proven the conjecture in the general case for graphs of bounded treewidth. We will not be discussing this result in any detail in this thesis. While the result is important it is not directly relevant to the approach taken here. Instead we will examine in more detail the result of Bodlaender et al [7] which showed that the conjecture holds if we also require our graphs to be chordal or of bounded chordality.

\subsubsection{Bodlaender, Heggernes and Telle}

In this thesis we will be proving an analogue to a result of Bodlaender, Heggernes and Telle [7]. These authors showed that recognisability equals definability for graphs of bounded treewidth and bounded chordality. We shall briefly examine this result. Actually they prove two results.

First they show that any recognisable family of connected edge-labeled chordal graphs of bounded treewidth is definable in a counting monadic second-order logic for graphs. This is the result we shall be following. Let $G$ be a graph. Paraphrasing from Bodlaender et al. [7] a perfect elimination ordering of $G$ is a linear ordering of the vertices of the graph so that the higher-numbered neighbours of any vertex form a clique. An orientation of the edges of a graph has the adjacent out-neighbours property if for every pair of edges 
$\{u, v\},\{u, w\}$ (for $u, v, w \in V(G)$ if $\{u, v\}$ is directed from $u$ to $v$ and $\{u, w\}$ is directed from $u$ to $w$ then $v$ and $w$ are adjacent in $G$.

Bodlaender et al. rely on the equivalency of the following statements for graphs.

i) $G$ is chordal.

ii) $G$ has a perfect elimination ordering.

iii) $G$ has a tree decomposition of optimal width where each bag induces a clique in $G$.

iv) There is an acyclic orientation of the edges of $G$ with the adjacent out-neighbours property.

This is a well-known result. They then show that given an acyclic orientation of the edges of $G$ with the adjacent out-neighbours property they can find a spanning tree $T$ for $G$ which can then be used to construct a tree decomposition of $G$. Essentially Bodlaender et al. show that constructing $T$ is definable in a counting monadic second-order logic essentially the same as the logic $\mathcal{C L}$ of Courcelle [15]. They then label the vertices of $T$ with the bounded elements of each bag of the associated tree decomposition. This results in a labeled tree $T_{L}$.

They are then able to apply, or as they say "mimic", Courcelle's Theorem for Trees (Theorem 3.3.1) to show that $T_{L}$ is recognisable if and only if it is definable by a statement $\phi$. Finally they show the quantifications over elements of $T_{L}$ directly translate to quantifications over the vertex and edge sets in $G$. This means that $\phi$ can be translated to sentence $\phi$ ' such that $\phi$ holds for the $T_{L}$ if and only if $\phi$ ' holds for $G$.

Bodlaender et al. [7] also prove a more general result for graphs of bounded 
chordality and bounded treewidth. This is not relevant to this thesis.

\subsubsection{A Converse for Matroids}

This leads to a question. Can one find and prove a converse to Hliněný's Theorem (Theorem 3.4.4) for matroids represented over a finite field of bounded branchwidth in a similar way to the various partial converses to Courcelle's Theorem for graphs? Answering this question will consume the rest of this thesis. We will show that one can establish a result for matroids represented over a finite field of bounded branchwidth that is an analogue to Bodlaender et al. [7] and thus a partial converse to Hliněný's Theorem [25]. For this we need to find a class of represented matroids that has the structure that we need. 


\section{Chapter 4}

\section{Modularity and Roundness}

In this chapter, we give some known results that will be background for what follows, in our search for the structure in matroids that we will need to prove a partial converse to Hliněný's Theorem [25]. Initially we are interested in the concept of modularity. This is both important in its own right and because it underlies supersolvability. Many of the results relating to modularity are due to Brylawski [12]. We are also interested in the roundness of matroids.

\subsection{Modularity}

Lemma 4.1.1 (Oxley [41]). Let $M$ be a matroid and let $F$ be a flat of $M$. Let $x \in E(M)$ and $x \notin F$. Then $\mathrm{cl}_{M / x}(F)=\mathrm{cl}_{M}(F \cup x)-x$.

Let $M$ be a matroid. Two flats $F_{1}$ and $F_{2}$ form a modular pair if $r_{M}\left(F_{1}\right)+$ $r_{M}\left(F_{2}\right)=r_{M}\left(F_{1} \cup F_{2}\right)+r_{M}\left(F_{1} \cap F_{2}\right)$. Furthermore a flat $F$ of $M$ is modular if $F$ and $X$ form a modular pair for every other flat $X$ of $M$.

Consider the following theorem of Brylawski [12]. The original was written in terms of lattice theory and has here been expressed in terms of matroids. 
Theorem 4.1.2 (Brylawski [12]). Let $M$ be a loopless matroid and let $F$ be a flat of $M$ then the following are equivalent

(i) Fis modular.

(ii) For all flats $Z$ of $M$ such that $F \cap Z=\emptyset$ we have $r_{M}(F)+r_{M}(Z)=$ $r_{M}(F \cup Z)$.

(iii) For all flats $Z$ of $M$ with $F \cap Z=\emptyset$ and $\mathrm{cl}_{M}(F \cup Z)=E(M)$ we have $r_{M}(F)+r_{M}(Z)=r_{M}(F \cup Z)$.

Proof. We first prove that (i) holds if and only if (ii) holds. Assume that $F$ is not modular. We show that in this case (ii) does not hold. Then there exists a flat $Z$ such that $r_{M}(F \cup Z)+r_{M}(F \cap Z)<r_{M}(F)+r_{M}(Z)$. This means that $r_{M}(F \cap Z)<r_{M}(F)+r_{M}(Z)-r_{M}(F \cup Z)$. We can assume that $r_{M}(F \cap Z) \neq 0$ since otherwise (ii) clearly does not hold.

Now let $I$ be a maximal independent set of $M$ contained in $F \cap Z$. Extend $I$ to a basis $B$ of $M \mid Z$. Let $Z^{\prime}=\operatorname{cl}_{M}(B-I)$. Then we make the following claims.

4.1.2.1. $r_{M}\left(Z^{\prime}\right)=r_{M}(Z)-|I|$.

Proof. Observe that $r_{M}\left(Z^{\prime}\right)=r_{M}\left(\operatorname{cl}_{M}(B-I)\right)=r_{M}(B-I)$. Since $B-I$ is independent we see that $r_{M}(B-I)=|B-I|=|B|-|I|$. Furthermore $r_{M}(B)=r_{M}(Z)$ since $B$ is a basis of $M \mid Z$. Hence $r_{M}\left(Z^{\prime}\right)=r_{M}(Z)-|I|$.

4.1.2.2. $r_{M}\left(F \cup Z^{\prime}\right)=r_{M}(F \cup Z)$.

Proof. Observe that $r_{M}\left(F \cup Z^{\prime}\right)=r_{M}\left(F \cup \mathrm{cl}_{M}(B-I)\right)=r_{M}(F \cup(B-I))=$ $r_{M}(F \cup B)$. However $r_{M}(F \cup B)=r_{M}(F \cup Z)$ since $B$ is a basis of $M \mid Z$ hence the result follows. 
4.1.2.3. $F \cap Z^{\prime}=\emptyset$.

Proof. Consider $x \in F$. We can see that there is no $y \in B-I$ such that $y$ is in $F \cap Z$. We note that $F \cap Z \subseteq F$. Suppose $x$ is in $F \cap Z^{\prime}$. Thus $x \in \operatorname{cl}_{M}(B-I)$. Now $F \cap Z^{\prime} \subseteq F \cap Z$, so $x \in \operatorname{cl}(I)$. Since $B$ is a basis of $M \mid Z$, we have $x \notin B$. Since $x \in \operatorname{cl}(B-I)$ and $x \in \operatorname{cl}(I)$, there are circuits $C$ and $C^{*}$ with $x \in C \subseteq(B-I) \cup x$ and $x \in C^{*} \subseteq I \cup x$. However, applying circuit elimination to $C$ and $C^{*}$ gives a circuit $C^{* *}$ contained in $B$, which is a contradiction. Thus $F \cap Z^{\prime}$ must be empty.

Observe that since $F \cap Z^{\prime}=\emptyset$ and $I$ is a maximal independent subset of $M$ contained in $F \cap Z$ then $r_{M}\left(F \cap Z^{\prime}\right)=0=r_{M}(F \cap Z)-|I|$.

4.1.2.4. $r_{M}\left(F \cap Z^{\prime}\right)<r_{M}(F)+r_{M}\left(Z^{\prime}\right)-r_{M}\left(F \cup Z^{\prime}\right)$.

Proof. Applying these previous claims we see that

$$
\begin{aligned}
& r_{M}(F)+r_{M}\left(Z^{\prime}\right) \\
= & r_{M}(F)+r_{M}(Z)-|I| \\
> & r_{M}(F \cup Z)+r_{M}(F \cap Z)-|I| \\
= & r_{M}\left(F \cup Z^{\prime}\right)+r_{M}\left(F \cap Z^{\prime}\right)
\end{aligned}
$$

This means that $r_{M}\left(F \cap Z^{\prime}\right)<r_{M}(F)+r_{M}\left(Z^{\prime}\right)-r_{M}\left(F \cup Z^{\prime}\right)$.

By the above claim it follows that $F$ must be modular if for all flats $Z$ of $M$ such that $F \cap Z=\emptyset$ we have $r_{M}(F)+r_{M}(Z)=r_{M}(F \cup Z)$.

We now prove that (ii) is equivalent to (iii). If (ii) holds then certainly the statement (iii) holds. So assume that (ii) does not hold. The goal is to show that (iii) does not hold. Let $F$ and $Z$ be flats with $F \cap Z=\emptyset$ such that 
$r_{M}(F)+r_{M}(Z)>r_{M}(F \cup Z)$. Let $I$ be a maximal independent set of $M$ contained in $F \cup Z$. Then we can extend $I$ to a basis $B$ of $M$. Let $J=B-I$ and let $Z^{\prime}=\operatorname{cl}_{M}(Z \cup J)$. Then we make the following claims.

4.1.2.5. $r_{M}\left(Z^{\prime}\right)=r_{M}(Z)+|J|$.

Proof. Observe that $r_{M}\left(Z^{\prime}\right)=r_{M}\left(\operatorname{cl}_{M}(Z \cup J)\right)=r_{M}(Z \cup J)$. By construction, $r_{M}((F \cup Z) \cup J)=r_{M}(F \cup Z)+r_{M}(J)$. Since $Z \subseteq F \cup Z$, we also have $r_{M}(Z \cup J)=r_{M}(Z)+r_{M}(J)$, that is $r_{M}(Z \cup J)=r_{M}(Z)+|J|$.

4.1.2.6. $r_{M}\left(F \cup Z^{\prime}\right)=r(M)$.

Proof. We can see that $B \subseteq F \cup Z^{\prime}$. Thus the result follows.

4.1.2.7. $F \cap Z^{\prime}=\emptyset$.

Proof. Observe that $j \notin \mathrm{cl}_{M}(F \cup Z \cup(J-\{j\}))$ for all $j \in J$. Otherwise we would have $j \in \operatorname{cl}_{M}(B-\{j\})$ which contradicts the fact that $B$ is independent.

Assume there is an $f \in F$ and that $f \in Z^{\prime}$. Then $f \in \operatorname{cl}_{M}(Z \cup J)$. This means that there is a circuit $C$ such that $f \in C \subseteq Z \cup J \cup\{f\}$. It follows that $C-\{f\} \nsubseteq Z$ since otherwise $f \in \operatorname{cl}_{M}(Z)$. Thus $C-\{f\} \subseteq Z \cup J$.

Now for some $j \in J$ it must be that $j \in C$. Hence $j \in \operatorname{cl}_{M}(C-\{j\})$. But $C-\{j\} \subseteq Z \cup(J-\{j\}) \cup\{f\} \subseteq F \cup Z \cup(J-\{j\})$. This means that $j \in \operatorname{cl}_{M}(C-\{j\}) \subseteq \operatorname{cl}_{M}(F \cup Z \cup(J-\{j\})$. This contradicts the observation at the beginning of this proof. This means that there can be no such $f \in F$ that is also in $Z^{\prime}$ and hence $F \cap Z^{\prime}=\emptyset$.

Given these claims we see that $r_{M}\left(F \cap Z^{\prime}\right)=0$ and $r_{M}\left(\mathrm{cl}_{M}\left(F \cup Z^{\prime}\right)\right)=r(M)$ yet $r_{M}(F)+r_{M}\left(Z^{\prime}\right)=r_{M}(F)+r_{M}(Z)+|J|>r_{M}\left(F \cup Z^{\prime}\right)=r(M)$. 
There is an alternative way of viewing modularity. The following is originally a result from Brylawski [12] also given in Oxley [41]. Let $L$ be a flat of a matroid $M$. Observe that $L$ is a line if $L$ is of rank 2 .

Lemma 4.1.3 (Brylawski [12]). Let $M$ be a loopless matroid. A hyperplane $H$ of $M$ is modular if and only if it intersects every line of $M$.

Proof. Let $H$ be a hyperplane of $M$. Assume that $H$ is modular. Let $L$ be a line of $M$. By definition $r_{M}(H)=r(M)-1$ and $r_{M}(L)=2$. Evidently $r_{M}(H \cup L) \leq r(M)$. We now have $r_{M}(H \cap L)=r_{M}(H)+r_{M}(L)-r_{M}(H \cup L) \geq$ $r(M)-1+2-r(M)=1$. As $r_{M}(H \cap L)>0$ it follows that $H \cap L \neq \emptyset$.

Now suppose $H$ is not modular. Our goal is to show that $H \cap L=\emptyset$ for some line $L$. Consider Theorem 4.1 .2 part (iii). There exists a flat $Z$ of $M$ such that $H \cap Z=\emptyset$ and $r_{M}(H \cup Z)=r_{M}(M)$ and yet $r_{M}(H \cup Z)<$ $r_{M}(H)+r_{M}(Z)$. Note that $r_{M}(Z) \geq 2$. Assume $r_{M}(Z)=k \geq 2$. If $r_{M}(Z)=2$ the result follows. Choose a maximal independent set $I$ of $M$ contained in $Z$ so that $|I|=r_{M}(Z)>2$. Let $\left\{z, z^{*}\right\}$ be a pair of elements in I. Then $Z^{*}=\operatorname{cl}\left(\left\{z, z^{*}\right\}\right) \subseteq Z$ and $r_{M}\left(Z^{*}\right)=2$. But we see that $H \cap Z^{*}=\emptyset$.

We call a matroid $M$ modular if for every two flats, $F_{1}$ and $F_{2}$ of $M$, then $F_{1}$ and $F_{2}$ form a modular pair. For example, consider the Fano plane. Every three point line of this matroid is a modular flat. Each three point line is a hyperplane of the matroid and intersects every other line of the matroid. We now give some useful well-known results.

Lemma 4.1.4. Let $M$ be a matroid. If $X, A, B \subseteq E(M)$ and $A \subseteq B$ then $r(X \cup B)-r(B) \leq r(X \cup A)-r(A)$.

Proof. By submodularity $r(X \cup A)+r(B) \geq r((X \cup A) \cup B)+r((X \cup A) \cap B)$. But observe that $A \subseteq(X \cup A) \cap B$. The result follows by rearranging the given inequality. 
Now consider the following well-known result.

Lemma 4.1.5. If $M$ is a matroid and $X \subseteq E(M)$ then the flats of $M \mid X$ are the set $\{F \cap X \mid F$ a flat of $M\}$.

We see that modularity extends to flats contained in other flats, as earlier shown by Brylawski [12].

Lemma 4.1.6 (Brylawski [12]). Let $M$ be a matroid. If $F_{1}$ is a modular flat in $M$ and $F_{2}$ is a modular flat in $M \mid F_{1}$, then $F_{2}$ is a modular flat in $M$.

Proof. We know that by submodularity $r_{M}\left(F_{2}\right)+r_{M}(Z) \geq r_{M}\left(F_{2} \cap Z\right)+$ $r_{M}\left(F_{2} \cup Z\right)$ for every $Z \subseteq E(M)$. Moreover by the modularity of $F_{1}$ it follows that $r_{M}\left(F_{1}\right)+r_{M}(Z)=r_{M}\left(F_{1} \cap Z\right)+r_{M}\left(F_{1} \cup Z\right)$ for every flat $Z$ in $M$. Now $F_{1} \cap Z$ is a flat of $M \mid F_{1}$. Also $F_{2}$ is modular in $M \mid F_{1}$ and $F_{2} \subseteq F_{1}$. Therefore it follows that $r_{M}\left(F_{1} \cap Z\right)+r_{M}\left(F_{2}\right)=r_{M}\left(\left(F_{1} \cap Z\right) \cap F_{2}\right)+r_{M}\left(\left(F_{1} \cap Z\right) \cup F_{2}\right)=$ $r_{M}\left(F_{2} \cap Z\right)+r_{M}\left(\left(F_{1} \cap Z\right) \cup F_{2}\right)$.

Observe that $\left(F_{1} \cap Z\right) \cup F_{2} \subseteq F_{1}$. Then by Lemma 4.1.4 we see that $r_{M}\left(\left(\left(F_{1} \cap Z\right) \cup F_{2}\right) \cup Z\right)-r_{M}\left(\left(F_{1} \cap Z\right) \cup F_{2}\right) \geq r_{M}\left(F_{1} \cup Z\right)-r\left(F_{1}\right)$. Thus $r_{M}\left(F_{2} \cup Z\right) \geq r_{M}\left(F_{1} \cup Z\right)-r_{M}\left(F_{1}\right)+r\left(\left(F_{1} \cap Z\right) \cup F_{2}\right)=r_{M}\left(F_{1}\right)+r_{M}(Z)-$ $r_{M}\left(F_{1} \cap Z\right)-r_{M}\left(F_{1}\right)+r_{M}\left(F_{2}\right)+r_{M}\left(F_{1} \cap Z\right)-r_{M}\left(F_{2} \cap Z\right)$.

Hence we see that $r_{M}\left(F_{2} \cup Z\right) \geq r\left(F_{2}\right)+r_{M}(Z)-r_{M}\left(F_{2} \cap Z\right)$. The result follows by application of this result and the fact about submodularity in the first paragraph of this proof.

Now we characterize modularity under the operations of intersection and union of flats.

Lemma 4.1.7 (Brylawski [12]). Let $M$ be a matroid. If $F$ and $G$ are modular flats in $M$ then so is $F \cap G$. 
Proof. By the Lemma 4.1.6 it suffices to prove that $F \cap G$ is modular in $M \mid F$.

Say $X$ is a flat of $M \mid F$. Observe also that $X \cap G=X \cap(F \cap G)$. As $G$ is modular in $M$, we see that $r(X)+r(G)=r(X \cup G)+r(X \cap G)$. Because of the result earlier in this paragraph $r(X)+r(G)=r(X \cup G)+r(X \cap(F \cap G))$. Hence $r(X \cup G)-r(G)=r(X)-r(X \cap(F \cap G))$.

Now by Lemma 4.1.4, $r(X \cup G)-r(G) \leq r(X \cup(F \cap G))-r(F \cap G)$. Therefore $r(X \cup(F \cap G))-r(F \cap G) \geq r(X)-r(X \cap(F \cap G))$.

Rearranging this inequality we obtain $r(X)+r(F \cap G) \leq r(X \cup(F \cap G))+$ $r(X \cap(F \cap G))$. But by submodularity of the rank function we know also that $r(X)+r(F \cap G) \geq r(X \cup(F \cap G))+r(X \cap(F \cap G))$. It follows that $r(X)+r(F \cap G)=r(X \cup(F \cap G))+r(X \cap(F \cap G))$.

In other words we see that $F \cap G$ is modular in $M \mid F$ and hence in $M$ by Lemma 4.1.6.

Let $M$ be a matroid. Let $T \subseteq E$ be a set. Then $T$ is a modular set if $r_{M}(T)+r_{M}(Z)=r_{M}(T \cup Z)+r_{M}(T \cap Z)$ for every flat $Z$ in $M$.

Lemma 4.1.8. Given a matroid $M$ and $F, X \subseteq E(M)$ then

i) $r_{M}\left(\operatorname{cl}_{M}(F) \cup X\right)=r_{M}(F \cup X)$.

ii) If $F$ is a modular set and $X$ is a flat then $r_{M}\left(\operatorname{cl}_{M}(F) \cap X\right)=r_{M}(F \cap X)$.

Proof. We shall prove each statement in turn.

i) This result is well-known. 
ii) Observe that $\operatorname{cl}_{M}(F) \cap X \supseteq F \cap X$. Then $r_{M}\left(\operatorname{cl}_{M}(F) \cap X\right) \geq r_{M}(F \cap X)$. Because $F$ is a modular set we also know that $r_{M}(F \cap X)=r_{M}(F)+$ $r_{M}(X)-r_{M}(F \cup X)$. But by the properties of the rank function $r_{M}$ we see that

$$
\begin{aligned}
& r_{M}\left(\mathrm{cl}_{M}(F) \cap X\right) \\
\leq & r_{M}\left(\mathrm{cl}_{M}(F)\right)+r(X)-r_{M}\left(\mathrm{cl}_{M}(F) \cup X\right) \\
= & r_{M}(F)+r_{M}(X)-r_{M}\left(\operatorname{cl}_{M}(F \cup X)\right) \\
= & r_{M}(F)+r_{M}(X)-r_{M}(F \cup X) \\
= & r_{M}(F \cap X)
\end{aligned}
$$

We recall from earlier that $r_{M}\left(\operatorname{cl}_{M}(F) \cap X\right) \geq r_{M}(F \cap X)$. Thus if $F$ is a modular set $r_{M}\left(\operatorname{cl}_{M}(F) \cap X\right)=r_{M}(F \cap X)$

Lemma 4.1.9. Let $T$ be a modular set for some matroid $M$. Then $\mathrm{cl}_{M}(T)$ is a modular flat in $M$.

Proof. This follows from the definition of modular set.

We can describe modularity under contraction of elements.

Theorem 4.1.10. Let $F$ be a modular flat of a matroid $M$. Then

i) if $x \in F$ then $F-x$ is modular in $M / x$.

ii) if $x \notin F$ then $\mathrm{cl}_{M / x}(F)$ is modular in $M / x$.

Proof. Suppose $x \in F$. We know from Oxley [41] (pp.100 - 106) that $S-x$ is a flat in $M / x$ for any flat $S$ in $M$ with $x \in S$. The rank in $M / x$ of $S-x$ 
is $r_{M}((S-x) \cup x)-r_{M}(\{x\})=r_{M}(S)-r_{M}(\{x\})$. If $x$ is not a loop then $r_{M}(\{x\})=1$ otherwise $r_{M}(\{x\})=0$.

i) By Oxley [41] $F-x$ is a flat of $M / x$. Observe that for every flat $S$ in $M$ the set $F-x$ satisfies $r_{M / x}(F-x)+r_{M / x}(S-x)=r_{M / x}((F-x) \cup$ $(S-x))+r_{M / x}((F-x) \cap(S-x))$.

ii) Suppose $x \notin \operatorname{cl}_{M}(F)$. Then $x \notin F$. We first prove that $F$ is a modular set of $M / x$. Given $F$ and any flat $S$ of $M$ observe that if $x$ is not a loop then the following hold:

- $r_{M / x}(F)=r_{M}(F \cup x)-1=r_{M}(F)+1-1=r_{M}(F)$

- $r_{M / x}(S-x)=r_{M}((S-x) \cup x)-1=r_{M}(S)-1$ provided $x \in S$

- $r_{M / x}(F \cup(S-x))=r_{M}((F \cup(S-x)) \cup x)-1=r_{M}(F \cup S)-1$

- $r_{M / x}(F \cap(S-x))=r_{M}((F \cap(S-x)) \cup x)-1=r_{M}((F \cup x) \cap S)-1=$ $r_{M}((F \cap S) \cup x)-1=r_{M}(F \cap S)$

We then see by modularity of $F$ in $M$ that $r_{M / x}(F)+r_{M / x}(S-x)=$ $r_{M}(F)+r_{M}(S)-1=r_{M}(F \cup S)+r_{M}(F \cap S)-1=r_{M}(F \cup S)-1+$ $r_{M}(F \cap S)=r_{M / x}(F \cup(S-x))+r_{M / x}(F \cap(S-x))$. We obtain a similar result if we let $x$ be a loop. (see above in part i) ). Given the above proof and Lemma 4.1.9 we see that $\mathrm{cl}_{M / x}(F)$ must be a modular flat of $M / x$. 
Let $M$ be a matroid and let $F$ be a flat in $M$. Then an $F$-strand is a set $Z \subseteq E(M)-F$ such that $\sqcap(F, Z)=1$. Remember that $\sqcap(F, Z)=$ $r_{M}(F)+r_{M}(Z)-r_{M}(F \cup Z)$.

Lemma 4.1.11. Let $M$ be a loopless matroid and $F$ be a flat of $M$. Then $F$ is modular if and only if for every $F$-strand $Z$ we have $F \cap \mathrm{cl}_{M}(Z) \neq \emptyset$.

Proof. First suppose that $F$ is modular. Then $r_{M}(F \cap X)=r_{M}(F)+r_{M}(X)-$ $r_{M}(F \cup X)$ for every flat $X$ in $M$. For every $F$-strand $Z$ we know that $\mathrm{cl}_{M}(Z)$ is a flat and so $r_{M}\left(F \cap \mathrm{cl}_{M}(Z)\right)=r_{M}(F)+r_{M}(Z)-r_{M}(F \cup Z)$. For each strand $Z$ we know by definition that $\sqcap(F, Z)=1$ hence $r_{M}\left(F \cap \mathrm{cl}_{M}(Z)\right)=1$. Thus $F \cap \operatorname{cl}_{M}(Z) \neq \emptyset$.

Suppose that $F$ is not a modular flat. By Theorem 4.1 .2 (ii) there exists a flat $Z$ such that $F \cap Z=\emptyset$ and $r_{M}(F)+r_{M}(Z)>r_{M}(F \cup Z)$. Say $t=r_{M}(F)+r_{M}(Z)-r_{M}(F \cup Z)$. Then $t \geq 1$. We complete the proof by induction on $t$. For any set $X \subseteq E(M)$, set $\sqcap(X, F)=r_{M}(F)+r_{M}(X)-r_{M}(F \cup X)$. The flat $F$ is not modular, so there is a flat $Z$, disjoint from $F$, with $\sqcap(Z, F)=t>0$. For any $z \in Z$, we have $0 \leq r_{M}(Z)-r_{M}(Z-z) \leq 1$ and $0 \leq r_{M}(F \cup Z)-r_{M}(F \cup(Z-z)) \leq 1$.

This observation and modularity give

$$
0 \leq r_{M}(Z)+r_{M}(F \cup(Z-z))-r_{M}(F \cup Z)-r_{M}(Z-z) \leq 1
$$

from which we get $\sqcap(Z, F)-1 \leq \sqcap(Z-z, F) \leq \sqcap(Z, F)$. Since $\sqcap(\emptyset, F)=0$, it follows that there is a subset $Z^{\prime}$ of $Z$ with $\sqcap\left(Z^{\prime}, F\right)=1$, as needed.

Next we show that we can describe modularity in another way that does not require mention of rank. This will be useful later when we need to define the 
modularity of flats in monadic second order logic.

Lemma 4.1.12. Let $M$ be a matroid and let $F, F^{*}$ be flats of $M$. Then the following hold.

i) If $F, F^{*}$ are a modular pair then, for every basis, Bu, of $F \cup F^{*}$, it follows that $B u \cap F$ is a basis of $F$ and $B u \cap F^{*}$ is a basis of $F^{*}$.

ii) If there exist bases, $B u$, of $F \cup F^{*}$ and $B n$, of $F \cap F^{*}$, such that $B n \subseteq B u$, where $B u \cap F$ is a basis of $F$ and $B u \cap F^{*}$ is a basis of $F^{*}$ then $F, F^{*}$ are a modular pair.

Proof. First we show that if $F$ and $F^{*}$ are a modular pair then, for every basis $B u$ of $F \cup F^{*}$, it follows that $B u \cap F$ is a basis of $F$ and $B u \cap F^{*}$ is a basis of $F^{*}$. Suppose not. Then suppose $B u$ is a basis of $F \cup F^{*}$ such that $B u \cap F$ is not a basis of $F$. Let $B$ be a basis of $F$. Then $|B u \cap F|<|B|$.

Now we can see that $|B u|=\left|(B u \cap F) \cup\left(B u \cap F^{*}\right)\right|=|B u \cap F|+\left|B u \cap F^{*}\right|-$ $\left|(B u \cap F) \cap\left(B u \cap F^{*}\right)\right|<|B|+\left|B^{*}\right|-\left|(B u \cap F) \cap\left(B u \cap F^{*}\right)\right|$. If $B n \subseteq B u$ then $|B n|=\left|(B n \cap F) \cap\left(B n \cap F^{*}\right)\right| \leq\left|(B u \cap F) \cap\left(B u \cap F^{*}\right)\right|$. But then $|B u|<|B|+\left|B^{*}\right|-|B n|$. Rearranging and remembering that these are bases we have $r_{M}(F)+r_{M}\left(F^{*}\right)>r_{M}\left(F \cup F^{*}\right)+r_{M}\left(F \cap F^{*}\right)$. Thus $F$ and $F^{*}$ are not a modular pair.

Next we show that if there exist bases $B u$, of $F \cup F^{*}$, and $B n$, of $F \cap F^{*}$, such that $B n \subseteq B u$, where $B u \cap F$ is a basis of $F$ and $B u \cap F^{*}$ is a basis of $F^{*}$ then $F$ and $F^{*}$ are a modular pair. If $B_{n}$ is a basis of $F \cap F *$ and if $B_{u}$ is a basis of $F \cup F *$, for which $B n \subseteq B u$, and $F \cap B u$ is a basis of $F$, and likewise for $F *$, then

$$
|F \cap B u|+|F * \cap B u|=|B u|+|B n| .
$$


Writing these terms as ranks gives exactly the equality that defines $(F, F *)$ as being a modular pair.

Let $M$ be a matroid and $F$ be a modular flat of $M$. Let $p \in F$ be an element of $F$. Then $p$ is necessary if there exists an $F$-strand $Z$ such that $\mathrm{cl}_{M}(Z) \cap F=\{p\}$. Otherwise $p$ is unnecessary. Next, let $F$ be a modular flat of $M$. Then we call $F$ a minimal modular flat if for all $x \in F$, the set $F-x$ is not modular in $M \backslash x$. Consider the following result.

Lemma 4.1.13. Let $M$ be a matroid. $F$ is a minimal modular flat of $M$ if and only if every point $p \in F$ is necessary.

Proof. Assume that every point in $F$ is necessary and let $p$ be such a point. Then we know there is a strand $Z$ such that $F \cap \mathrm{cl}_{M}(Z)=\{p\}$. If we remove $p$ then we see that $(F-p) \cap \mathrm{cl}_{M \backslash p}(Z)=\emptyset$. That is there is a strand $Z$ that does not meet $F-p$ hence $F-p$ is not modular in $M \backslash p$. This is true for all points $p$ in $F$.

Assume then that there is some point $x$ in $F$ that is unnecessary. Then there does not exist an $F$-strand $Z$ such that $F \cap \mathrm{cl}_{M}(Z)=\{x\}$. If we remove $x$ from $F$ and remember $F$ is modular then by Lemma $4.1 .10 F-x$ remains modular in $M \backslash x$. Hence $F$ can not be minimally modular.

We give a counter-example showing that the closure of the union of modular flats is not necessarily modular. This also shows that the union of modular flats is not necessarily modular.

Example 4.1.14. Consider the question whether if $M$ is a simple matroid and $F$ and $G$ are two flats of $M$ then if $F$ and $G$ are modular is $\operatorname{cl}_{M}(F \cup G)$ modular? This is false. Observe $M\left(K_{4}\right)$ in Figure 4.1 and let $F$ and $G$ be single points of rank 1 each on different lines. All rank-1 flats in matroids are modular, but not all lines are. 


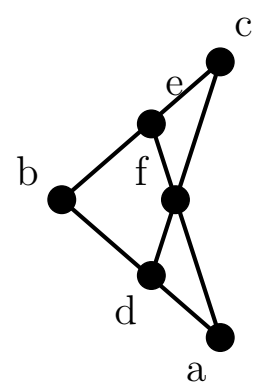

Figure 4.1: $M\left(K_{4}\right)$ - An example where $\operatorname{cl}_{M}(F \cup G)$ is not modular

\subsection{Roundness}

The notion of roundness for matroids was introduced under the name of nonsplitting by Kung [35]. In this section we give some standard well-known properties of round matroids. Roundness can be defined in several equivalent ways, as the next theorem shows.

Theorem 4.2.1. Let $M$ be a matroid. Then the following are equivalent:

i) Every cocircuit of $M$ is spanning.

ii) $M$ has no vertical $k$-separations.

iii) $E(M)$ is not the union of two proper flats.

iv) $M$ is not the union of two hyperplanes.

v) $E(M)$ has no partition into two non-spanning sets.

Proof. First we show that Property i) implies Property ii). If there exists a $k$-separation which is vertical then there exists a cocircuit which is not spanning. Let $(A, B)$ be a $k$-separation which is vertical. Then both $A$ and $B$ are not spanning. But if $A$ is not spanning it is contained in a maximal non spanning set, that is, in a hyperplane. Hence $B$ must contain a cocircuit. This cocircuit can not be spanning else $B$ would be.

Next we show that Property ii) implies iii). By definition $M$ has no vertical $k$-separations if and only if for every $k$-separation $(A, B)$ of $M$ either $A$ or $B$ 
is spanning. But then by definition there are no partitions $(A, B)$ of $E(M)$ such that both $A$ and $B$ are non-spanning. Given this suppose there exist two proper flats $F_{1}$ and $F_{2}$ such that $E(M)=F_{1} \cup F_{2}$. But $F_{1}$ and $F_{2}$ are both non-spanning since they are proper flats.

Any subset of $F_{1}$ and $F_{2}$ must also be non-spanning. So consider $F_{1} \backslash F_{2}$ and $F_{2} \backslash F_{1}$. These sets are non-spanning but by definition $F_{1} \backslash F_{2} \cup F_{2} \backslash F_{1}=E(M)$ since $F_{1} \cup F_{2}=E(M)$. Also by definition $F_{1} \backslash F_{2} \cap F_{2} \backslash F_{1}$ is empty. Thus there exists a partition of $E(M)$ into two non-spanning sets. So we see that if $M$ has no vertical $k$-separations then $E(M)$ has no partition into two nonspanning sets and so $E(M)$ can not be written as the union of two proper flats.

Next we show that Property iii) implies Property iv). By definition a hyperplane is a proper flat. If $E(M)$ can not be written as the union of two proper flats $M$ certainly can not be the union of two hyperplanes.

Now, we assert that Property iv) implies Property v). Suppose there exists a partition of $E(M)$ into non-spanning sets. Consider one of these partitions $(A, B)$. Then $A$ is contained by a hyperplane as is $B$. Call these $H_{A}$ and $H_{B} . H_{A} \cup H_{B}$ contains $A \cup B=E(M)$. Hence $H_{A} \cup H_{B}=E(M)$ and so clearly $M$ is the union of two hyperplanes.

Finally we see that Property v) implies Property i). Suppose there is a nonspanning cocircuit $C *$. Then $C *$ and $E(M)-C *$ (which is a hyperplane) give a partition into two non-spanning sets.

We define a matroid to be round if it has no vertical $k$-separations. A flat $F$ is a round flat if $M \mid F$ is a round matroid. We now give some properties of roundness in matroids.

Lemma 4.2.2. Let $M$ be a round matroid and let $x$ be any element in $E(M)$. Then $M / x$ is round. 
Proof. By Theorem 4.2.1 every cocircuit of $M$ is spanning. But by Oxley [41] the cocircuits of $M / x$ are exactly the cocircuits of $M$ that do not contain $x$. These cocircuits are spanning with respect to $M$ hence the closure of each is equal to $E(M)$. These cocircuits clearly remain spanning with respect to $M / x$. As a result every cocircuit of $M / x$ is spanning hence, by Theorem 4.2.1, $M / x$ must be round.

Lemma 4.2.3. Let $M$ be a matroid and let $F$ be a subset of $E(M)$. If $x \notin \mathrm{cl}_{M}(F)$ then $(M / x)|F=M| F$.

Proof. If $x \notin \operatorname{cl}_{M}(F)$ then clearly $F \subseteq \operatorname{cl}_{M}(F) \subseteq E(M)-\{x\}$. Thus $(M / x)|F=M| F$ since by Oxley [41][Proposition 3.1.11] $\mathrm{cl}_{M / x}(F)=\mathrm{cl}_{M}(F \cup$ $x)-\{x\}$ and so $r_{M / x}(F)=r_{M}(F \cup x)-1=r_{M}(F)+1-1=r_{M}(F)$.

We now show that complete graphs have round cycle matroids. This is a well-known result.

Lemma 4.2.4. $M\left(K_{n}\right)$ is round for any $n$.

Proof. Let $(A, B)$ be a $k$-separation of $M\left(K_{n}\right)$ for some $n$. Assume it is vertical. If there exists a vertex, $v$, not incident with any edge in $A$, then all edges incident with $v$ are in $B$. This means that every edge is in $B$ or is in a triangle with 2 edges in $B$. This in turn implies that $B$ spans $A$ which is, of course, a contradiction.

Now, by symmetry, $A$ and $B$ are incident with all vertices. Let $G$ be the graph $K_{n}$. If $G[A]$ is not connected then $G[B]$ is, and in this case, $B$ contains a spanning tree, so $A \subseteq \operatorname{cl}(B)$. This is again a contradiction. Similarly, if $G[B]$ is not connected. This means that no $k$-separation of $M\left(K_{n}\right)$ for any $n$ can be vertical and thus $M\left(K_{n}\right)$ is round for any $n$. 
The next lemma is proved in Whittle [48].

Lemma 4.2.5. Let $H$ be a modular hyperplane of the matroid $M$, and let $\left\{c_{1}, c_{2}, \ldots, c_{n}\right\}$ be a basis for the cocircuit complementary to $M$. Let $B^{\prime}=\left\{\operatorname{cl}_{M}\left(\left\{c_{i}, c_{j}\right\}\right) \cap H: 1 \leq i<j \leq n\right\}$. Then $M \mid B^{\prime} \cong M\left(K_{n}\right)$.

Lemma 4.2.6. Let $F$ be a rank $t$ modular flat of the round matroid $M$. Then there is a spanning set $Z \subseteq F$ such that $M \mid Z \cong M\left(K_{t+1}\right)$.

Proof. Let $k=r(M)-r_{M}(F)$. We shall prove this result by induction on $k$. Observe that if $k=1$ then $F$ is a hyperplane. In this case Lemma 4.2.5 applies [48]) and the result holds. So suppose that $k>1$. Now hypothesize that the result holds for $k-1$ provided the matroid is round.

Consider some element $x \in E(M)-\mathrm{cl}_{M}(F)$. Note that, of course, $F=$ $\mathrm{cl}_{M}(F)$. From Lemma 4.2.2 we know that if $M$ is round then $M / x$ is round. By Lemma 4.1.9 and Theorem 4.1.10 if $x \notin \mathrm{cl}_{M}(F)$, and so $x \notin F$, then $F$ is a modular set of $M / x$ which means that $\operatorname{cl}_{M / x}(F)$ is a modular flat of $M / x$. Furthermore, we see that $F \subseteq \mathrm{cl}_{M / x}(F)$ because $x \notin F$. Moreover, we know from Oxley [41] that $r(M / x)=r(M)-1$. Given that $x \notin F$ this means that $r(M / x)-r_{M / x}\left(\operatorname{cl}_{M / x}(F)\right)=r(M)-1-r_{M}(F)=\left(r(M)-r_{M}(F)\right)-1=k-1$.

Thus by the inductive hypothesis there is a subset $Z$ of $\mathrm{cl}_{M / x}(F)$ such that $(M / x) \mid Z$ is isomorphic to $M\left(K_{t+1}\right)$. Suppose that $Z \nsubseteq F$ which means that $r_{M / x}(Z-F)>0$. Because $F$ is a modular set of $M / x$, we see that $r_{M / x}(Z-F)=r_{M / x}((Z-F) \cup F)+r_{M / x}((Z-F) \cap F)-r_{M / x}(F)$. But $r_{M / x}((Z-F) \cup F)=r_{M / x}\left((Z-F) \cup \operatorname{cl}_{M / x}(F)\right)=r_{M / x}(F)$ by Lemma 4.1.8 and because $Z-F \subseteq \mathrm{cl}_{M / x}(F)$. Also $r_{M / x}((Z-F) \cap F)=0$. As a result, we see that $r_{M / x}(Z-F)=r_{M / x}(F)+0-r_{M / x}(F)=0$. This contradicts our earlier supposition that $Z \nsubseteq F$. Thus, it must be that $Z \subseteq F$. 
By Lemma 4.2.3 we know that if $x$ is not a member of $\operatorname{cl}_{M}(F)$ then $(M / x) \mid F=$ $M \mid F$. Which means that $(M / x)|Z=M| Z$ because $Z \subseteq F$. Therefore, there is a subset $Z$ of $F$ such that $(M / x)|Z=M| Z \cong M\left(K_{t+1}\right)$.

Next consider the following lemma which shows when sets containing round sets are themselves round. We say that a flat $S$ of $M$ is round if $M \mid S$ is a round matroid.

Lemma 4.2.7. Let $M$ be a matroid and $S$ and $T$ be sets in $M$. If $S$ is round in $M$ then

(i) $\operatorname{cl}_{M}(S)$ is round in $M$.

(ii) if $T \supseteq S$ and $r_{M}(T)=r_{M}(S)$ then $T$ is round in $M$.

Proof. (i) Suppose $\operatorname{cl}_{M}(S)$ is not round in $M$. Then $M \mid \operatorname{cl}_{M}(S)$ has a vertical separation $(A, B)$. But $r_{M}\left(\operatorname{cl}_{M}(S)\right)=r_{M}(S)$ and because $(A, B)$ is vertical we know without loss of generality that $r_{M}(A)<$ $r_{M}\left(\operatorname{cl}_{M}(S)\right)=r_{M}(S)$. This clearly remains true for $M \mid S$.

(ii) Suppose $T$ contains $S$ and has the same rank as $S$ but is not round. Then $M \mid T$ contains a separation $(A, B)$ such that without loss of generality $\operatorname{cl}_{M \mid T}(A)=\mathrm{cl}_{M}(A) \subsetneq \mathrm{cl}_{M}(T)=\mathrm{cl}_{M \mid T}(T)$. But then $r_{M}(A)<$ $r(T)=r(S)$ hence $(A, B)$ is vertical in $M \mid S$.

The following is a corollary of Lemma 4.2.6.

Corollary 4.2.8. Let $M$ be a matroid. If $M$ is round then all modular flats of $M$ are round flats. 
Proof. This result follows directly from Lemma 4.2.6. Each modular flat $F$ has a spanning subset $Z \subseteq F$ which is by this lemma isomorphic to the matroid of a complete graph. Observe that the cycle matroid of a complete graph is round. By Lemma 4.2.7 the flat $F$, which contains $Z$, must be round. 


\section{Chapter 5}

\section{Chordality in Graphs}

In this chapter we review some well-known results about chordality in graphs. Much of this is available in Bondy and Murty [9].

\subsection{Chordal Graphs}

Let $G=(V, E)$ be a graph. Let $C$ be a cycle of $G$. Then a chord of $C$ is an edge $e \in E(G)-C$ of $G$, not parallel to any edge of $C$, such that the vertices incident with $e$ are in $V(C)$.

Lemma 5.1.1. Let $G=(V, E)$ be a simple graph and $C$ be a cycle of $G$ such that $|C|>3$. Then $e$ is a chord of $C$ if and only if there is a partition $\left(C_{1}, C_{2}\right)$ of $C$ such that $C_{1} \cup\{e\}$ and $C_{2} \cup\{e\}$ are cycles.

A subgraph $H$ of $G$ is induced by a set $X \subseteq V(G)$ if $H=(X, E(X))$. We say in this case that $X$ induces $H$. Let $G[X]$ denote the subgraph induced by $X . E(X)$ is the set of edges of $G[X]$. Remember that we denote by $\omega(H)$ the number of connected components of a graph $H$. Let $v$ be a vertex of $G$. Then $v$ is a cut vertex if $\omega(G-v)>\omega(G)$. Next $S \subseteq V(G)$ is a vertex cut if the $\omega(G[V(G)-S])>\omega(G)$. A vertex cut $S$ is minimal if $S-\{v\}$ is not 
a vertex cut for any vertex $v$ in $S$

A clique is a subgraph of $G$ that is isomorphic to $K_{t}$ for some $t$. Thus a clique is not just a set of vertices, although we often simplify and say that $V$ is a clique meaning that $G[V]$ is a clique. A clique $X$ of $G$ is a maximal clique if there is no vertex $v$ we can add to $X$ such that $X \cup\{v\}$ is a clique of $G$. A clique cut is a vertex cut of $G$ that induces a clique. A vertex $v$ in $G$ is a simplicial vertex if $\mathcal{N}_{G}(v)$ induces a clique.

Lemma 5.1.2. Let $G$ be a connected graph. Let $v$ be a simplicial vertex of $G$. Then $G-v$ is connected.

Proof. Suppose that $G-v$ is not connected. Then there are at least two components $C_{1}, C_{2}$ in $G-v$. Because $G$ is connected there must be a path from the vertices in $C_{1}$ to $v$ and from $C_{2}$ to $v$. Thus there is an edge from $v$ to a vertex $u$ in $C_{1}$ and from $v$ to a vertex $w$ in $C_{2}$. Because $v$ is a simplicial vertex there is an edge $\{u, w\}$. But this contradicts that $C_{1}$ and $C_{2}$ are disconnected. Thus $G-v$ is connected.

Next we state two results from Bondy and Murty [9]. A graph $G$ is chordal if, for every cycle $C$, such that $|C| \geq 3, C$ has a chord.

Theorem 5.1.3 (Bondy and Murty [9]). Let $G$ be a connected chordal graph. Let $S$ be a minimal vertex cut of $G$. Then $S$ is a clique cut.

Let $G$ be a graph and let $S$ be a vertex cut of $G$. If $G_{i}(1 \leq i \leq p)$ are the components of $G-S$ then the $S$-components of $G$ are the induced subgraphs $G_{i} \cup S(1 \leq i \leq p)$.

Theorem 5.1.4 (Bondy and Murty [9]). Let $G$ be a connected chordal graph. Let $V_{1}$ be a maximal clique of $G$. The maximal cliques of $G$ can be arranged in a sequence $\left(V_{1}, V_{2}, \ldots, V_{k}\right)$, starting with $V_{1}$, such that $V_{j} \cap\left(\bigcup_{i=1}^{j-1} V_{i}\right)$ is a clique of $G$, for $2 \leq j \leq k$. 
Let $G$ be a graph. We can therefore define a simplicial decomposition of $G$ to be a sequence $\left(V_{1}, V_{2}, \ldots, V_{k}\right)$ of maximal cliques such that $V_{j} \cap\left(\bigcup_{i=1}^{j-1} V_{i}\right)$ is a clique of $G(2 \leq j \leq k)$.

Lemma 5.1.5. Let $G$ be a chordal graph. Then any induced subgraph $H$ of $G$ is also chordal.

Proof. Let $C$ be a cycle of $H$ with at least 4 edges. If $C$ has a chord $e$ in $G$ then $e$ is in $H$ since $H$ is induced.

Corollary 5.1.6. Let $G$ be a connected chordal graph. Let $S$ be a minimal vertex cut of $G$. Then the $S$-components of $G$ are also chordal.

Proof. By definition earlier the $S$-components of $G$ are induced subgraphs of $G$. Hence by Lemma 5.1 .5 the $S$-components of $G$ must be chordal.

If $u, v$ are vertices of $G$ then the notation $u v$ means the edge from $u$ to $v$. Next we state a well-known graph-theoretic result.

Lemma 5.1.7. Let $e=u v$ be an edge of the graph $G$. Then the cycles of G/e are sets of the form:

i) $C$, where $C \cup\{e\}$ is a cycle of $G$; or

ii) $C$ where $C$ is a cycle of $G$ such that $V(C)$ contains at most one of $u$ and $v$.

Given this we see the following.

Lemma 5.1.8. Let e be an edge of the chordal graph $G$. Then $G /$ e is chordal. 
Proof. Let $e=u v$. Let $C$ be a cycle of $G / e$ with $|C| \geq 4$. If $C$ is of type i) of Lemma 5.1.7 then $C \cup\{e\}$ is a cycle of $G$. Now $|C \cup\{e\}| \geq 5$ so by definition $C \cup\{e\}$ has a chord $f=x y$ in $G$. Then, by Lemma 5.1.1, we have a partition $C_{1}, C_{2}$ of $C \cup\{e\}$, where $\left|C_{1}\right|,\left|C_{2}\right| \geq 2$, such that $C_{1} \cup\{f\}, C_{2} \cup\{f\}$ are cycles of $G$.

We may assume that $e \in C_{1}$. By Lemma 5.1.7 $\left(C_{1} \cup\{f\}\right)-\{e\}$ is a cycle of $G / e$. Also the set of vertices of $C_{2} \cup\{f\}$ contains at most one of $u$ and $v$. Then $C_{2} \cup\{f\}$ is cycle of $G / e$. Now $f$ is chord of $C$ in $G / e$ unless $\left|\left(C_{1} \cup\{f\}\right)-\{e\}\right|=2$.

Assume that $\left|\left(C_{1} \cup\{f\}\right)-\{e\}\right|=2$. Then $\left|C_{2} \cup\{f\}\right| \geq 4$. As $G$ is chordal, $C_{2} \cup\{f\}$ has a chord $g$ in $G$. Observe then that $g$ is a chord of $C_{2} \cup\{e\}$. Say by Lemma 5.1.1 that $C_{1}^{*}, C_{2}^{*}$ are the partitions of $C_{2} \cup\{e\}$ such that $C_{1}^{*} \cup\{g\}, C_{2}^{*} \cup\{g\}$ are both cycles in $G$ and $e \in C_{1}^{*}$. Then $C_{1}^{*} \supsetneq C_{1}$. This means that $\left|C_{1}^{*} \cup\{g\}\right| \geq 4$. It follows that $g$ is a chord of $C$ in $G / e$.

Now assume that $C$ is a cycle of type ii) of Lemma 5.1.7. Then at most $u$ or $v$ is in $V(C)$. Since $|C| \geq 4$ we know that $C$ contains a chord $f$. Then by Lemma 5.1.1 there is a partition $C_{1}, C_{2}$ of $C$ such that $C_{1} \cup\{f\}$ and $C_{2} \cup\{f\}$ are cycles of $G$. Then $V\left(C_{1} \cup\{f\}\right)$ and $V\left(C_{2} \cup\{f\}\right)$ contain at most one element of $u$ and $v$. By Lemma 5.1.7 $C_{1} \cup\{f\}$ and $C_{2} \cup\{f\}$ are cycles of $G / e$. Thus $f$ is a chord of $G / e$.

Next we introduce the concept of a tree of cliques. A clique tree of $G$ is a tree $T$ whose vertices are the maximal cliques of $G$ and for each vertex $x \in V(G)$, the set of maximal cliques containing $x$ induces a subtree of $T$. The reduced clique graph of $G$ (see [23, 21]), denoted $C_{r}(G)$, has as its vertices the set of maximal cliques of $G$. An edge joins the cliques $U$ and $V$ if $U \cap V$ is a minimal separator of $G$ with the property that $U-V$ and $V-U$ are in different components of $G \backslash(U \cap V)$. Each edge of $C_{r}(G)$ is labeled with the order of the separation formed by its minimal separator. 
We can use an algorithm such as that of Kruskal [34] to find a maximum weighted spanning tree of $C_{r}(G)$ [23, 21]. Interestingly, Habib et al. [23, 21] have shown that for graphs with the very particular structure of $C_{r}(G)$ there is a more efficient linear-time algorithm to compute a maximum weighted spanning tree of $C_{r}(G)$.

Lemma 5.1.9 (Habib et al. [23, 21]). Let $G=(V, E)$ be a chordal graph. Let $C_{r}(G)$ be its reduced clique graph. Let $T=(C, F)$ be a spanning tree of $C_{r}(G)$. Then $T$ is a clique tree of $G$ if and only if $T$ is a maximum weighted spanning tree of $C_{r}(G)$.

Lemma 5.1.10. Let $G$ be a connected chordal graph. Let $T_{C}(G)$ be a clique tree of $G$. Let $X_{i}, X_{j}, X_{k}$ be maximal cliques of $T_{C}(G)$. If $X_{j}$ lies on a path of $T_{C}(G)$ between $X_{i}$ and $X_{k}$ then $X_{i} \cap X_{k} \subseteq X_{j}$.

Proof. Let $x$ be a vertex of $G$. Suppose $x \in X_{i}$ and $x \in X_{k}$. By definition of a clique tree the set of maximal cliques containing $x$ induces a subtree of $T_{C}(G)$. But this must include every maximal clique on a path between $X_{i}$ and $X_{k}$ and hence $X_{j}$ since trees are connected. Thus if $x \in X_{i} \cap X_{k}$ then $x \in X_{j}$.

Theorem 5.1.11. Let $G$ be a connected chordal graph. Let $T(G)$ be a maximum weighted spanning tree of $C_{r}(G)$. Then $T(G)$ is a minimum width tree decomposition of $G$.

Proof. Recall the properties of a tree decomposition from the preliminaries in Chapter 2 Section 2.5. By Lemma 5.1.9 $T(G)$ is a clique tree of $G$. Then consider the properties of a tree decomposition. Every vertex $x$ of $G$ is in a maximal clique of $G$. Since $T(G)$ is a spanning tree every maximal clique is included and every vertex $x$ in $G$ is in some maximal clique of $T(G)$. Thus Property (1) of a tree decomposition holds.

Next by definition every edge $e$ of $G$ is in a maximal clique of $G$. Again since $T(G)$ is spanning every edge must be included in some maximal clique of 
$T(G)$ and so Property (2) holds. Now let $X_{i}, X_{j}, X_{k}$ be maximal cliques of $T(G)$. Then by Lemma 5.1 .10 if $X_{j}$ lies on the path of $T(G)$ between $X_{i}$ and $X_{k}$ then $X_{i} \cap X_{k} \subseteq X_{j}$. Thus interpolation holds and Property (3) is satisfied.

Finally, let $t$ be the cardinality of the largest maximal clique of $G$. By definition every tree decomposition of $G$ must have width at least $t$. However, by definition $T(G)$ must have width exactly $t$. Thus $T(G)$ has minimum width.

Thus chordal graphs have an associated tree decomposition where the bags are cliques.

Theorem 5.1.12 (Dirac [18]). Let $G$ be a connected chordal graph. If $G$ is not complete then it contains two non-adjacent simplicial vertices.

Proof. Assume that $|C| \geq 2$ for each maximal clique $C$ of $G$. Let $C_{r}(G)$ be the reduced clique tree for $G$. By Theorem 5.1.9 we know we can find a maximum weighted spanning tree $T(G)$ for $C_{r}(G)$. Then by Theorem 5.1.11 we know that this is a minimum width tree decomposition of $G$. Any tree with more than 1 vertex has two leaves.

In this case each leaf of $T(G)$ is a clique. At least one of the vertices of this clique will be simplicial. Since there are two separate cliques, each clique has at least two vertices and we can choose any of these as our simplicial vertex such that it is not adjacent to any of those in the other clique.

So chordality may be the sort of structure we want. But what does it mean in terms of matroids? 


\subsection{Cycle Matroids of Chordal Graphs}

First consider matroids for chordal graphs. Recall Lemma 5.1.2. This can be restated as if $v$ is a vertex of a graph $G$ then, if $v$ is simplicial, $v$ can not be a cut vertex.

Lemma 5.2.1. Let $G$ be a connected simple graph and let $M(G)$ be the cycle matroid of $G$. Let $v$ be a vertex which is not a cut vertex. Then $E(G-v)$ spans a hyperplane in $M(G)$.

Proof. The subgraph $G-v$ is connected so $r_{M(G)}(E(G-v))=|V(G-v)|-1=$ $|V(G)|-2$. Moreover, $G$ is connected so that $r(M(G))=|V(G)|-1$. This means that $r_{M(G)}(E(G-v))=r(M(G))-1$. This means that $E(G-v)$ is contained in a hyperplane.

Now say that $e \in E(G)-E(G-v)$. Then $e$ is incident with $v$. This means that $r_{M(G)}(E(G-v) \cup\{e\})=|V(E(G-v) \cup\{e\})|-1=|V(G)|-1>$ $r_{M(G)}(E(G-v))$. Thus $E(G-v)$ spans the hyperplane it is contained in.

Lemma 5.2.2. Let $G$ be a graph, let $M(G)$ be the cycle matroid of $G$, and let $v$ a vertex of $G$. If $v$ is simplicial then $E(G-v)$ is a modular hyperplane in $M(G)$.

Proof. By Lemmas 5.1.2 and 5.2.1 we see that $E(G-v)$ is a hyperplane. Let $C^{*}$ be the set of edges incident with $v$ so that $C^{*}=E(G)-E(G-v)$. Let $a_{1}=$ $v w_{1}, a_{2}=v w_{2}$ be edges in $C^{*}$. Because $v$ is a simplicial vertex there is an edge $b=w_{1} w_{2}$. Note that $\left\{a_{1}, a_{2}, b\right\}$ is a triangle in $G$ hence $b \in \operatorname{cl}_{M(G)}\left(\left\{a_{1}, a_{2}\right\}\right)$. Thus we have shown that for every pair of elements $a_{1}, a_{2} \in C^{*}$ there is an element $b \in E(G-v)$ such that $b$ belongs to $\operatorname{cl}\left(\left\{a_{1}, a_{2}\right\}\right)$. This means that $E(G-v)$ must be modular by Lemma 4.1.3.

Now consider the following well-known result. 
Lemma 5.2.3. Let $G$ be a 2-connected graph and $B$ be a bond of $G$. Then $E(G)-B$ induces a connected graph if and only if $B$ is the set of edges incident with a vertex of $G$.

Given this we prove the following lemma.

Lemma 5.2.4. Let $M$ be a 2-connected matroid and $H$ be a modular hyperplane of $M$. Then $M \mid H$ is 2-connected.

Proof. Assume that $M \mid H$ is not connected. We may assume that $M$ is simple. Let $C^{*}=E(M)-H$. By Lemma 4.1 .3 for all $x, y \in C^{*}$, there is an element $e_{x y}$ such that $e_{x y} \in \operatorname{cl}(\{x, y\}) \cap H$. If $r\left(C^{*}\right)=1$, then $M$ is not connected. So suppose that $r\left(C^{*}\right)>1$. Say $\left\{c_{1}, \ldots, c_{n}\right\}$ is a basis for $C^{*}$. Let $H_{1}$ be the component of $M \mid H$ containing $e_{c_{1} c_{2}}$ and let $H_{2}=H-H_{1}$. We have $r(H)=r\left(H_{1}\right)+r\left(H_{2}\right)$.

5.2.4.1. $e_{c_{1} c_{i}} \in H_{1}$ for all $i \in\{2, \ldots, n\}$.

Proof. If not there exists a $j$ such that $e_{c_{1} c_{j}} \in H_{2}$. Then there is a line through $e_{c_{1} c_{2}}$ and $e_{c_{1} c_{j}}$. Again by Lemma 4.1 .3 we know there must be an element $e_{c_{2} c_{j}} \in \operatorname{cl}\left(\left\{e_{c_{1} c_{2}}, e_{c_{1} c_{j}}\right\}\right) \cap H$. Thus we know that $e_{c_{1} c_{2}}, e_{c_{1} c_{j}}, e_{c_{2} c_{j}}$ is a circuit. But this contradicts the fact that circuits must be contained in a single component. From this the result follows.

Now consider $\operatorname{cl}\left(H_{1} \cup\left\{c_{1}\right\}\right)$. Since $\left\{c_{1}, c_{i}, e_{c_{1} c_{i}}\right\}$ is a triangle then $c_{i} \in$ $\operatorname{cl}\left(H_{1} \cup\left\{c_{i}\right\}\right)$ for all $i \in\{2, \ldots, n\}$. But $\left\{c_{1}, \ldots, c_{n}\right\}$ is a basis for $M \mid C^{*}$ as we have seen hence $\operatorname{cl}\left(H_{1} \cup\left\{c_{1}\right\}\right) \supseteq C^{*}$. Therefore $r\left(H_{1} \cup\left\{c_{1}\right\}\right)=r\left(H_{1}\right)+1$ which means that $r\left(H_{1} \cup C^{*}\right)=r\left(H_{1}\right)+1$. Now $r(M)=r(H)+1=$ $r\left(H_{1}\right)+r\left(H_{2}\right)+1=r\left(H_{1} \cup C^{*}\right)+r\left(H_{2}\right)$.

This means that $H_{1} \cup C^{*}$ and $H_{2}$ must be separators of $M$. Therefore $M$ is not connected. The result follows by the contrapositive. 
Lemma 5.2.5. Let $G$ be a 2-connected graph and let $M(G)$ be a cycle matroid of $G$. Let $H$ be a modular hyperplane of $M(G)$ then $E(G-H)$ is incident with a simplicial vertex in $G$.

Proof. By Lemma 5.2.4 $M(G) \mid H$ is 2-connected. Now $E(G)-H$ is a bond. By Lemma 5.2 .3 there is a vertex $v \in G$ such that $E(G)-H$ is the set of edges of $G$ incident with $v$.

To show that $v$ is simplicial we need to show that every pair of neighbours of $v$ is connected by an edge. Let $w_{1}, w_{2}$ be neighbours of $v$ in $G$. Let $e_{1}=v w_{1}$ and $e_{2}=v w_{2}$. Then because $H$ is a modular hyperplane there is an element $h \in H$ such that $h \in \operatorname{cl}_{M(G)}\left(\left\{e_{1}, e_{2}\right\}\right)$. Hence $\left\{e_{1}, e_{2}, h\right\}$ is a triangle in $G$ which means that $h=\left\{w_{1}, w_{2}\right\}$.

Theorem 5.2.6. Let $G$ be a 2-connected graph. Then $v$ is a simplicial vertex if and only if $E(G-v)$ is a modular hyperplane of $M(G)$.

Proof. By Lemmas 5.2.2 and 5.2.5. 


\section{Chapter 6}

\section{Chordality in Matroids}

In this chapter we engage in a discussion of when and how the concept of chordality can be applied to matroids. We examine binary matroids, graphic matroids and matroids in general. Apart from the definitions for the different types of chordality in matroids most of the work in this chapter is original.

\subsection{Some Background}

\subsubsection{Alternative Definitions of Chordality}

First, we present some alternative definitions of chordality for matroids. These are not necessarily new but are derived from existing concepts of chordality for graphs. As it turns out these definitions are all equivalent for graphic matroids and are equivalent to chordality in the usual sense.

\section{Weak Chordality}

Let $M$ be a simple matroid. $M$ is weakly chordal if for every circuit $C$, $|C|>3$, there exist subsets $C_{1}, C_{2}$ of $C$ with $C_{1}, C_{2} \subseteq C=C_{1} \cup C_{2}$ and $e \in E(M)-C$ such that $C_{1} \cup e$ and $C_{2} \cup e$ are also circuits of $M$. 


\section{Bonin Chordality}

$M$ is Bonin chordal [10] if for every circuit $C,|C|>3$, there exist subsets $C_{1}, C_{2}$ of $C$ with $C_{1}, C_{2} \subseteq C=C_{1} \cup C_{2}$ and $e \in E(M)-C$ such that $C_{1} \cup e$ and $C_{2} \cup e$ are also circuits of $M$ and $\left|C_{1} \cup e\right|,\left|C_{2} \cup e\right|<|C|$.

\section{T-Chordality}

A circuit of $M$ is a triangle if it has cardinality of exactly 3. $M$ is T-chordal if for every circuit $C,|C|>3$, there is a pair of elements $c, c^{\prime} \in C$ and an element $e \in E(M)-C$ such that $\left\{c, c^{\prime}, e\right\}$ is a triangle.

\section{C-Chordality}

$M$ is $C$-chordal [14] if for every circuit $C,|C|>3$, there are circuits $C_{1}, C_{2}$ and an $e \notin C$ such that $C_{1} \cap C_{2}=e$ and $C=C_{1} \triangle C_{2}$. We call $e$ a $C$-chord of $C$.

We can rephrase this as follows. $M$ is C-chordal if for every circuit $C,|C|>3$, there is a partition $C_{1}, C_{2}$ of $C$, and an element $e \notin C$, such that $C_{1} \cup e$ and $C_{2} \cup e$ are circuits.

\section{Strong Chordality}

$M$ is strongly chordal if for every circuit $C,|C|>3$, there exist $c, c^{\prime} \in C$ and $e \in E(M)-C$ such that $\left\{c, c^{\prime}, e\right\}$ and $\left(C-\left\{c, c^{\prime}\right\}\right) \cup e$ are also circuits of $M$. 


\subsubsection{The History of These Definitions}

Weak chordality was introduced for binary matroids by Barahona and Groetschel [3] where they referred to weakly chordal for matroids as "chordal". Here we do not restrict our meaning to the class of binary matroids.

Bonin and de Mier [10] define a circuit $C$ to be "chordal" if there are circuits $C_{1}$ and $C_{2}$ of $M$ and an element $x \in C_{1} \cap C_{2}$ such that $\left|C_{1}\right|,\left|C_{2}\right|<|C|$ and $C=\left(C_{1} \cup C_{2}\right)-x$. For us a matroid is Bonin chordal if and only if each circuit of size at least 4 is chordal in their sense.

Cordovil et al [14] analyze matroids and graphs with a view to understanding how a chordal graph is like a supersolvable matroid. We shall be using some of their results later in Chapter 7. In particular they show that a binary supersolvable matroid is chordal in their sense but the converse does not hold. In the process of establishing this result they give another definition of chordality in matroids from Barahona and Groetschel [3].

Let $M$ be an arbitrary matroid. Cordovil et al [14] say that a circuit $C$ of $M$ has a "chord" $e$ if there are two circuits $C_{1}$ and $C_{2}$ such that $C_{1} \cap C_{2}=e$ and $C=C_{1} \triangle C_{2}$. In this case, we say that the chord $e$ "splits" the circuit $C$ into the circuits $C_{1}$ and $C_{2}$. We say that a matroid is $i$-chordal if every circuit with at least $i$ elements has a chord. The matroids we call $C$-chordal are matroids which are 4-chordal in the sense of [14].

Finally, we are left with an extension of our definition of T-chordality in matroids which we call strong chordality. As seen earlier a matroid $M$ is strongly chordal if for every circuit $C$ of $M$ such that $|C|>3$ there exist elements $c_{1}, c_{2}$ of $C$ and an element $e \notin C$ such that both $\left\{c_{1}, c_{2}, e\right\}$ and $\left(C-\left\{c_{1}, c_{2}\right\}\right) \cup e$ are circuits. If a matroid is strongly chordal we call it "chordal" for short. 
Observe that our use of the terms strongly chordal and weakly chordal are not at all similar to the use of these terms with graphs. Our usage is a direct definition of a type of chordal not an addition of features to chordal. A graph is weakly chordal if it is chordal and is (anti-hole, hole)-free [24]. A graph is strongly chordal if it is chordal and every cycle of even length has an odd chord [11.

All this results in the following theorem.

Theorem 6.1.1. Let $G$ be a graph. Then the following are equivalent

i) $M(G)$ is weakly chordal

ii) $M(G)$ is Bonin chordal

iii) $M(G)$ is T-chordal

iv) $M(G)$ is C-chordal

v) $M(G)$ is strongly chordal

Proof. By definition $M(G)$ is a graphic matroid. The result follows from Corollary 6.3.6 and Theorems 6.2.1 and 6.2.2. See the next sections of this chapter.

\subsection{Chordality for Matroids in General}

We now consider how these definitions of chordality for matroids interrelate. The equivalence of these definitions of chordality does not hold for matroids 
in general. However, observe that strong chordality for matroids does imply all the other types and weak chordality for matroids is implied by all the other types.

Theorem 6.2.1. Let $M$ be a matroid. Let $M$ be strongly chordal. Then the following is true.

i) $M$ is weakly chordal; and

ii) $M$ is Bonin chordal; and

iii) $M$ is T-chordal; and

iv) $M$ is C-chordal.

Proof. We shall deal with each in turn. First let $C$ be a circuit of $M$. Assume $|C|>3$. We know there exist $c, c^{\prime} \in C$ and $e \in E(M)-C$ so that $\left\{c, c^{\prime}, e\right\}$ and $\left(C-\left\{c, c^{\prime}\right\}\right) \cup e$ are circuits. Let $C_{1}=\left\{c, c^{\prime}\right\}$ then $C_{2}=C-\left\{c, c^{\prime}\right\}$. Then we see easily that $C_{1} \cup e$ and $C_{2} \cup e$ are both circuits of $M$. Since this is true for every circuit $C$ of $M$ we see that $M$ is also weakly chordal.

Moreover $\left|C_{1} \cup e\right|=\left|\left\{c, c^{\prime}, e\right\}\right|=3<|C|$. Also because $\left\{c, c^{\prime}\right\} \subseteq C$ and $e \notin C$ we see that $\left|C_{2} \cup e\right|=\left|\left(C-\left\{c, c^{\prime}\right\}\right) \cup e\right|=(|C|-2)+1=|C|-1<|C|$. Thus $\left|C_{1} \cup e\right|,\left|C_{2} \cup e\right|<|C|$. Since this is true for every circuit $C(|C|>3)$ it follows that if $M$ is strongly chordal it must also be Bonin chordal.

It is trivial to see that $M$ must be T-chordal. Finally, $\left\{c, c^{\prime}, e\right\} \cap\left(C-\left\{c, c^{\prime}\right\}\right) \cup$ $e=e$. Also $\left\{c, c^{\prime}, e\right\}-\left(C-\left\{c, c^{\prime}\right\}\right) \cup e=\left\{c, c^{\prime}\right\}$ and $\left(C-\left\{c, c^{\prime}\right\}\right) \cup e-\left\{c, c^{\prime}, e\right\}=$ $C-\left\{c, c^{\prime}\right\}$ and $\left\{c, c^{\prime}\right\} \cup\left(C-\left\{c, c^{\prime}\right\}\right)=C$. Thus $\left\{c, c^{\prime}, e\right\} \triangle\left(C-\left\{c, c^{\prime}\right\}\right) \cup e=C$. So $M$ is C-chordal.

Theorem 6.2.2. Let $M$ be a matroid. Then the following are true. 
i) if $M$ is strongly chordal then $M$ is weakly chordal.

ii) If $M$ is Bonin chordal then $M$ is weakly chordal.

iii) If $M$ is T-chordal then $M$ is weakly chordal

iv) If $M$ is $C$-chordal then $M$ is weakly chordal

Proof. We shall deal with each in turn. We have seen already that if $M$ is strongly chordal then $M$ is weakly chordal. If $M$ is Bonin chordal then for every circuit $C$ of $M,|C|>3$, there are two subsets of $C, C_{1}$ and $C_{2}$, and an element $e \notin C$, such that $C_{1} \cup e$ and $C_{2} \cup e$ are circuits and $\left|C_{1} \cup e\right|,\left|C_{2} \cup e\right|<|C|$. But then it is clear that $M$ must be weakly chordal.

Next, consider a circuit $C$ of $M$ such that $|C|>3$. Then if $M$ is T-chordal there are elements $c_{1}, c_{2} \in C$ and an element $e \in E(M)-C$ such that $\left\{c_{1}, c_{2}, e\right\}$ is a triangle. Observe that $r_{M}(C)=|C|-1$ since $C$ is a circuit so a minimal dependent set. Also we see that $r_{M}(C \cup\{e\})=|C|-1$. Thus $C-\left\{c_{1}\right\}$ is independent which means that $\left|C-\left\{c_{1}\right\}\right|=|C|-1$. This means that $C-\left\{c_{1}\right\}$ must span $C \cup\{e\}$ and so $e \in \operatorname{cl}_{M}\left(C-\left\{c_{1}\right\}\right)$.

6.2.2.1 (Oxley [41]). If $x \in \mathrm{cl}_{M}(S)-S$ then there exists a circuit $D \subseteq S \cup x$ such that $x \in D$.

This means that there is a circuit $C_{2} \cup\{e\}$ such that $C_{2} \subseteq C-\left\{c_{1}\right\}$. Thus $M$ is weakly chordal.

Finally, let $C$ be any circuit of $M$. Then $M$ is C-chordal we know that there exist circuits $C_{1}, C_{2}$ and an element $e \notin C$ such that $C_{1} \cap C_{2}=e$ and $C_{1} \triangle C_{2}=C$. So let $C *_{1}=C_{1}-C_{2}=C_{1}-\{e\}$ and $C *_{2}=C_{2}-C_{1}=C_{2}-\{e\}$. 
$C=C *_{1} \cup C *_{2}$ by definition. Finally we know that $C *_{1} \cup\{e\}=C_{1}$ and $C *_{2} \cup\{e\}=C_{2}$ are circuits. Thus $M$ is weakly chordal.

\subsection{Chordality in Binary Matroids}

In this section we see that for binary matroids, and so graphic matroids, our definitions for chordality in matroids become equivalent.

First, let $C$ be a circuit of the matroid $M$. We say that $C$ is T-chordal if there exists $e \in E(M)$ and $c_{1}, c_{2} \in C$ such that $\left\{c_{1}, c_{2}, e\right\}$ is a triangle. Observe that a simple matroid $M$ is T-chordal if and only if every circuit of $M$ is T-chordal in this sense.

Lemma 6.3.1. Let $M$ be a simple matroid. If $C$ is a circuit of $M$ with distinct subsets $C_{1}, C_{2}$ and an element $e \in E(M)$ such that $C_{1} \cup\{e\}$ and $C_{2} \cup\{e\}$ are circuits then $C=C_{1} \cup C_{2}$.

Proof. By circuit elimination there is a circuit $C_{3} \subseteq\left(C_{1} \cup C_{2}\right)-\{e\}$. But $C_{1} \cup C_{2} \subseteq C$ and $C_{3} \subseteq\left(C_{1} \cup C_{2}\right)$. Since circuits can not contain other circuits it follows that $C_{1} \cup C_{2}=C$.

Lemma 6.3.2. Let $M$ be a simple binary matroid which is weakly chordal. Let $C$ be a circuit of $M$ with $|C|>3$. Then there is an element $e$ and a partition $C_{1}, C_{2}$ of $C$ such that $C_{1} \cup$ e and $C_{2} \cup$ e are circuits.

Proof. By the definition of weak chordality, we know there exist subsets $C_{1}$ and $C_{2}$ and an element $e \in E(M)-C$ such that $C_{1} \cup e$ and $C_{2} \cup e$ are circuits. We further know that because $M$ is binary then for any two distinct circuits $X, Y$ the symmetric difference $(X-Y) \cup(Y-X)$ contains a circuit. But then $\left(\left(C_{1} \cup e\right)-\left(C_{2} \cup e\right)\right) \cup\left(\left(C_{2} \cup e\right)-\left(C_{1} \cup e\right)\right)=\left(C_{1}-C_{2}\right) \cup\left(C_{2}-C_{1}\right)$ contains a circuit $C^{\prime}$. But because $C_{1}, C_{2}$ are subsets of $C$ we see that $C^{\prime} \subseteq$ $\left(C_{1}-C_{2}\right) \cup\left(C_{2}-C_{1}\right) \subseteq C$. Since circuits are minimally dependent we see that $\left(C_{1}-C_{2}\right) \cup\left(C_{2}-C_{1}\right)=C$. This implies that $C_{1}, C_{2}$ is a partition of $C$. 
Lemma 6.3.3. Let $M$ be a simple binary matroid and let $M$ be weakly chordal. Then $M$ is T-chordal.

Proof. We shall prove this by induction on the size of $C$. Let $C$ be a circuit of $M$ such that $|C|>3$. As a base case assume that $|C|=4$. Since $M$ is weakly chordal we know that there exist $C_{1}, C_{2} \subseteq C=C_{1} \cup C_{2}$ and some $e \notin C$ such that $C_{1} \cup e$ and $C_{2} \cup e$ are circuits. Since $M$ is binary we know from Lemma 6.3 .2 that $C_{1}, C_{2}$ is a partition of $C$. But then $\left|C_{1}\right|,\left|C_{2}\right|<|C|$. Since $M$ is simple $\left|C_{1}\right|,\left|C_{2}\right| \neq 1$. Hence both $C_{1}$ and $C_{2}$ must be of cardinality 2 . Then the result follows.

Assume for induction that $C$ is a circuit of size $n>4$ and that all circuits of size less than $n$ are T-chordal. By Lemma 6.3.2 there is an $e$ and a partition of $\left(C_{1}, C_{2}\right)$ of $C$ such that $C_{1} \cup e$ and $C_{2} \cup e$ are circuits.

Observe that $\left|C_{1} \cup e\right| \geq 4$. Since $C_{1} \cup e$ is $T$-chordal by hypothesis, there is some element $y \in C_{1} \cup e$, pair $c, c^{\prime}$ in $\left(C_{1} \cup e\right)-\{y\}$ and element $f$ such that $\left\{c, c^{\prime}, f\right\}$ is a circuit. We know that $\left\{c, c^{\prime}\right\} \subseteq C_{1} \cup e$. We further know that $\{e\} \nsubseteq\left\{c, c^{\prime}\right\}$ because $e \in E(M)-C$. Also $M$ is simple so there are no parallel elements. Hence $\left\{c, c^{\prime}\right\} \subseteq C_{1} \subseteq C$ and $\left\{c, c^{\prime}\right\} \subseteq\left(C_{1} \cup e\right)-\{y\} \subseteq C-\{y\}$.

We have been given $f$ already so we see that there is an element $e$, pair $\left\{c, c^{\prime}\right\} \subseteq C-\{e\}$ and element $f$ such that $\left\{c, c^{\prime}, f\right\}$ is a circuit. Hence $C$ is T-chordal if $C_{1} \cup e$ has this property. Since this is true for all $C,|C|>3$, we see that $M$ must be T-chordal.

Lemma 6.3.4. Let $M$ be a simple matroid. If $M$ is binary, then $M$ is strongly chordal if and only if $M$ is weakly chordal.

Proof. By Lemma 6.2.1 if $M$ is strongly chordal then it is weakly chordal. So consider the converse.

Let $M$ be binary and weakly chordal. Then by Lemma 6.3.2 we know that 
for every circuit of $M$ call this $C$ there is a partition $C_{1}, D_{1}$ and some element $e \in E(M)$ such that $C_{1} \cup e$ and $D_{1} \cup e$ are also circuits of $M$.

By Lemma 6.3.3 $C$ is T-chordal. Thus $C$ contains a pair $\left\{c, c^{\prime}\right\}$ and there exists an element $f \notin C$ such that $\left\{c, c^{\prime}, f\right\}$ is a circuit. Now consider the set $\left(C-\left\{c, c^{\prime}\right\}\right) \cup f$.

By Oxley [41, Theorem 9.1.2] since $C$ and $\left\{c, c^{\prime}, f\right\}$ are circuits of $M$ then $C \triangle\left\{c, c^{\prime}, f\right\}$ is a disjoint union of circuits. But $C \triangle\left\{c, c^{\prime}, f\right\}=\left(C-\left\{c, c^{\prime}\right\}\right) \cup f$. This symmetric difference can be partitioned into circuits. Assume there is more than one circuit. Consider a circuit that does not contain $f$. Observe that this circuit is a subset of $C$ yet it can not be $C$ since it does not contain $c$ or $c^{\prime}$. This is a contradiction. Thus $\left(C-\left\{c, c^{\prime}\right\}\right) \cup f$ must contain a single circuit and thus itself be a circuit of $M$.

Thus we see that if $M$ is simple, binary and weakly chordal for every circuit $C$ with cardinality greater than 3 there must exist a pair $c, c^{\prime}$ and element $e \notin C$ such that both $\left\{c, c^{\prime}, f\right\}$ and $\left(C-\left\{c, c^{\prime}\right\}\right) \cup f$ are circuits and hence $M$ is strongly chordal.

Theorem 6.3.5. Let $M$ be a binary matroid. Then the following are equivalent:

i) $M$ is weakly chordal.

ii) $M$ is Bonin chordal.

iii) $M$ is T-chordal.

iv) $M$ is C-chordal. 
v) $M$ is strongly chordal.

Proof. This follows from Lemma 6.2.2, Lemma 6.2.1 and Lemma 6.3.4.

Corollary 6.3.6. Let $M$ be a graphic matroid. Then the following are equivalent:

i) $M$ is weakly chordal.

ii) $M$ is Bonin chordal.

iii) $M$ is T-chordal.

iv) $M$ is C-chordal.

v) $M$ is strongly chordal.

Proof. This is a corollary of Theorem 6.3.5 since every graphic matroid is binary.

\subsection{Further Discussion}

We now look at how chordality applies to matroids in general. In turn we consider the known implications, the known non-implications and the open questions. 


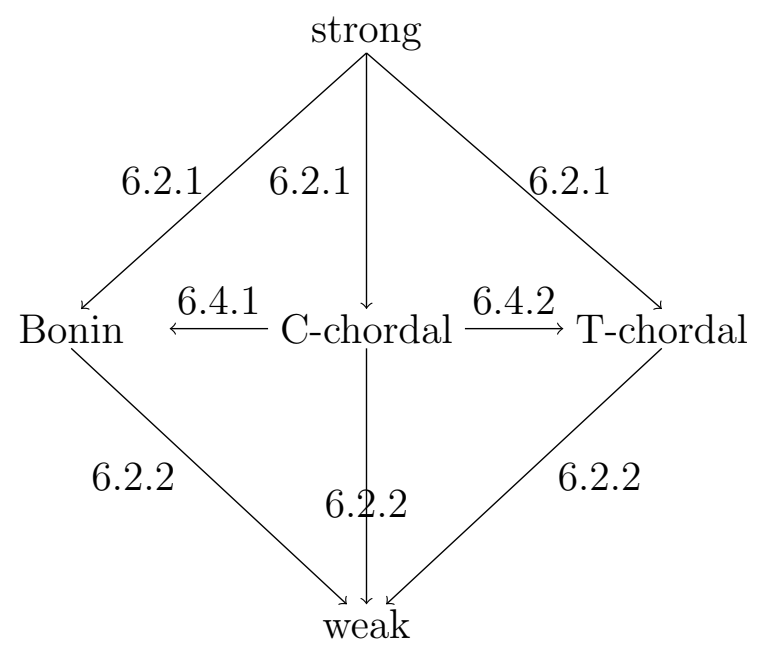

Figure 6.1: How the different implications of chordality in matroids relate. The arrows indicate the direction of implication. The edge labels refer to the Lemmas that establish the implications.

\subsubsection{Known Implications}

We saw earlier that strong chordality implies Bonin chordality, T-chordality, C-chordality and weak chordality in general matroids. In addition weak chordality is implied by all the other types. We also know that the following results about C-chordality hold. See the accompanying digraph in Figure 6.1.

Lemma 6.4.1. Let $M$ be a simple matroid. If $M$ is $C$-chordal then it is Bonin chordal.

Proof. Let $C$ be any circuit of $M$. Since $M$ is C-chordal we know that there exist circuits $C_{1}, C_{2}$ and $e \notin C$ such that $C_{1} \cap C_{2}=e$ and $C_{1} \triangle C_{2}=C$. Let $C *_{1}=C_{1}-C_{2}$ and $C *_{2}=C_{2}-C_{1}$. Then clearly $C *_{1} \cup e=C_{1}$ and $C *_{2} \cup e=C_{2}$ are circuits. Moreover $\left|C *_{1}\right|<|C|-1$ and $\left|C *_{2}\right|<|C|-1$ so $\left|C *_{1} \cup e\right|,\left|C *_{2} \cup e\right|<|C|$. Thus $M$ must be Bonin chordal.

Lemma 6.4.2. Let $M$ be a simple matroid. If $M$ is $C$-chordal then it is T-chordal.

Proof. Let $C$ be a circuit of $M$ such that $|C|>3$. Amongst all the elements $x \notin C$ of $M$, such that there exists a subset $C_{1} \subseteq C$ and $C_{1} \cup\{x\}$ is a circuit, 
choose $e$ such that $\left|C_{1} \cup\{e\}\right|$ is minimal. If $\left|C_{1} \cup\{e\}\right|=3$ the lemma holds. So assume $\left|C_{1} \cup\{e\}\right|>3$.

By the definition of C-chordality there is a partition $C_{1}^{\prime}, C_{2}^{\prime}$ of $C_{1}$ and an element $f$ of $M$ such that $C_{1}^{\prime} \cup f$ and $C_{2}^{\prime} \cup f$ are circuits. Observe that $f \notin C$ otherwise $C_{1}^{\prime} \cup f \subset C_{1} \cup e$ which can not be. We may assume that $e \notin C_{1}^{\prime}$. Then $C_{1}^{\prime} \subseteq C$ and $\left|C_{1}^{\prime} \cup\{f\}\right|<\left|C_{1} \cup\{f\}\right|$. This contradicts our requirement that $e$ be selected so that $\left|C_{1}\right|$ is minimal.

These results show that C-chordality is a fairly strong type of chordality for matroids. The question might then be are C-chordality and strong chordality equivalent? We discuss this later.

\subsubsection{Known Non-implications}

We know quite a lot about what does not hold in the relationships between the different types of chordality for general matroids. Working our way up consider how weak chordality relates to the stronger varieties of chordality. As one can see weak chordality does not imply strong chordality nor Bonin chordality nor T-chordality nor C-chordality. See the accompanying digraph in Figure 6.2.

We will use the matroid $U_{3,5}$ in the following four examples. A key feature of this matroid is that it does not allow triangles. Given low rank, almost all our definitions of chordality in matroids, except weak chordality, force the existence of triangles, as we will see.

Example 6.4.3. Weak chordality does not imply strong chordality for general matroids. There exists at least one matroid that is weakly chordal but not strongly chordal. Consider $U_{3,5}$ as in Figure 6.3 . 


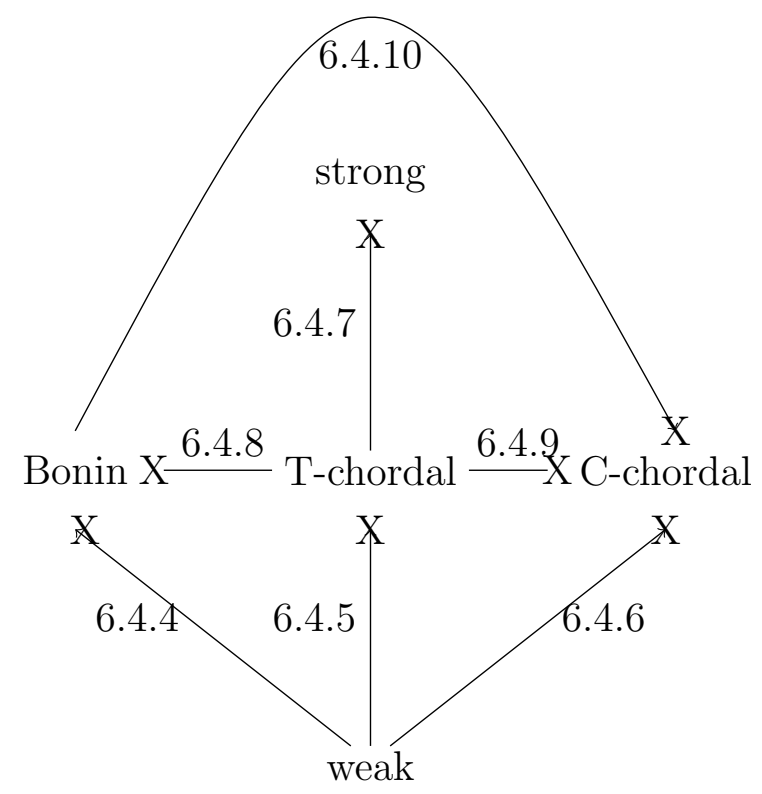

Figure 6.2: How the different non-implications of chordality in matroids relate. $A-\mathrm{X} B$ means " $A$ does not imply $B$ ". The edge labels indicate counterexamples for each case.

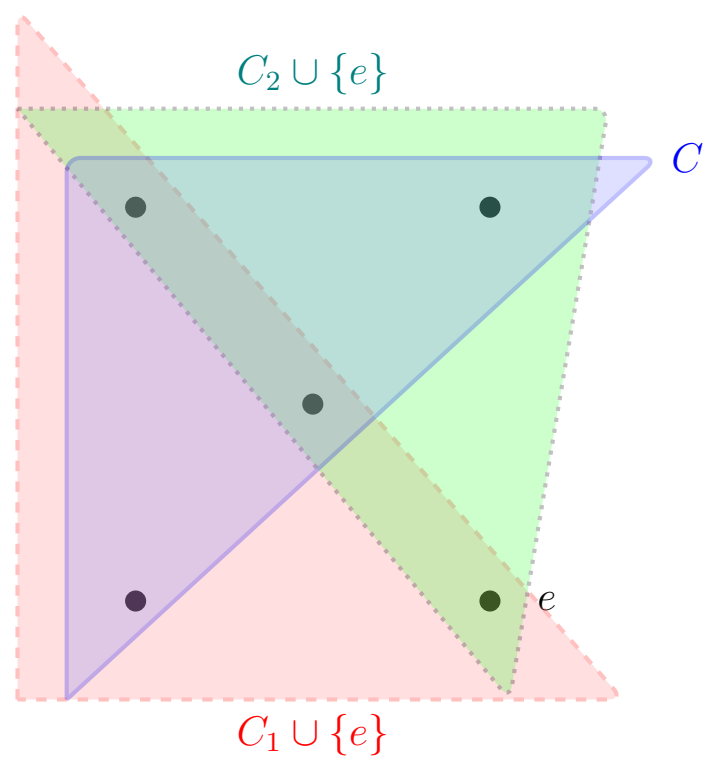

Figure 6.3: $U_{3,5}$ - the uniform matroid of 5 points on the plane 
Observe that $U_{3,5}$ is weakly chordal. For $U_{3,5}$ any 4 points form a circuit $C$. We can let $C_{1}$ be any 3 points of $C$ and let $C_{2}$ be another 3 points so that $C_{1} \cup C_{2}=C$. Let $e$ be the fifth point not in $C$. $C_{1} \cup e$ and $C_{2} \cup e$ are circuits.

But if we take any two points $c, c^{\prime}$ from $C$ then $\left\{c, c^{\prime}, e\right\}$ is not a circuit since it is an independent set. Hence $U_{3,5}$ is not strongly chordal.

Example 6.4.4. Weak chordality does not imply Bonin chordality in general matroids. Consider the matroid $U_{3,5}$ in Figure 6.3. Take any circuit $C$ of this matroid (which will have exactly 4 elements). We can choose two subsets $C_{1}, C_{2}$ of $C$ with $\left|C_{1}\right|=3=\left|C_{2}\right|$. Then we can choose some other element $e \in E-C$. Then $\left|C_{1} \cup e\right|=4=\left|C_{2} \cup e\right|$ and hence $C_{1} \cup e$ and $C_{2} \cup e$ are both also circuits. Thus $U_{3,5}$ is weakly chordal. However $\left|C_{1} \cup e\right|=\left|C_{2} \cup e\right|=4=|C|$. Thus this matroid is not Bonin chordal.

Example 6.4.5. Weak chordality does not imply T-chordality for general matroids. Consider the example matroid $U_{3,5}$ in Figure 6.3. Sets of size 3 can never be circuits in $U_{3,5}$. This will in fact be true for any choice of $C$. Thus while $U_{3,5}$ is weakly chordal it can not be T-chordal.

Example 6.4.6. Weak chordality does not imply C-chordality for general matroids. Observe $U_{3,5}$ in Figure 6.3. Clearly $C_{1} \cup e$ and $C_{2} \cup e$ are circuits so this example is weakly chordal. Now if $C_{1}^{*}=C_{1} \cup\{e\}$ and $C_{2}^{*}=C_{2} \cup\{e\}$ then clearly from observation $\left|C_{1}^{*} \cap C_{2}^{*}\right|>1$. Hence $C_{1}^{*} \cap C_{2}^{*} \neq\{e\}$. (Also from observation we can see that $C \neq C_{1}^{*} \triangle C_{2}^{*}$ ). This will be true for all choices of $C_{1}, C_{2}$. Thus this example is not $\mathrm{C}$-chordal.

Then consider T-chordality. As one can see T-chordality does not imply strong chordality nor Bonin chordality nor C-chordality for general matroids.

Example 6.4.7. T-chordality does not imply strong chordality for general matroids. Let $M$ be a T-chordal matroid. Then for every $C$, where $|C|>3$, 
there exist $c, c^{\prime} \in C$ and $e \notin C$ such that $\left\{c, c^{\prime}, e\right\}$ is a circuit. But for $M$ to be strongly chordal it must be that $\left(C-\left\{c, c^{\prime}\right\}\right) \cup e$ is also a circuit.

Consider $L_{2,3}$ as in Figure 6.4. Label the points on the 3-point line $c, d, e$ and the two other points $a, b$. Then $\{a, b, c, d\}$ is a circuit of size 4 (call this circuit $C$ ). Thus we know there exist two elements in $C$ and one outside whose union is a triangle.

Well clearly that is true since we can choose $\{c, d\}$ from $C$ and the remaining outside element $e$ which gives us $\{c, d, e\}$ which is the 3 -element and so a circuit.

However, observe that $(C-\{c, d\}) \cup\{e\}=\{a, b, e\}$ which is a basis not a circuit. This is true no matter how we select the two elements from $C$ since we are also constrained to choose two points on the 3 point line. Thus we can see that this matroid can not be strongly chordal.

Example 6.4.8. T-chordality does not imply Bonin chordality in general matroids. Consider again the rank 3 matroid $L_{2,3}$ in Figure 6.4. Label the elements on the 3 element line $c, d, e$ and the elements on the two element line $a, b$. Observe, as before, that $C=\{a, b, c, d\}$ is a circuit. This matroid is T-chordal because of the set $\{c, d, e\}$. However this matroid is not Bonin chordal because all circuits other than $\{c, d, e\}$ have cardinality 4.

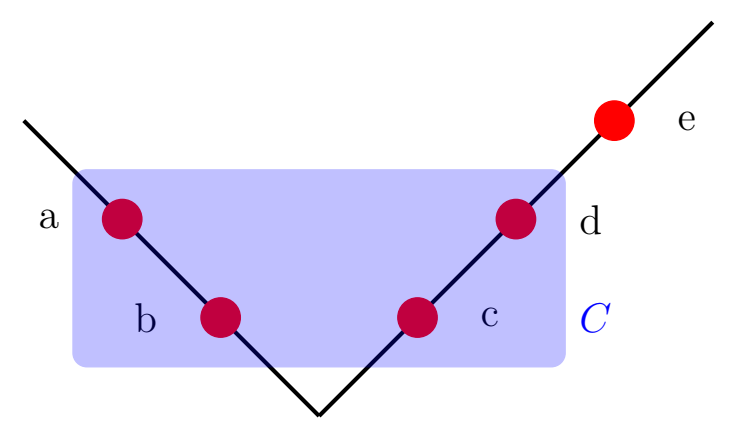

Figure 6.4: $L_{2,3}$ - the rank 3 matroid with two lines one with 3 points and one with 2 points 
Example 6.4.9. T-chordality does not imply C-chordality for general matroids. Consider again the example $L_{2,3}$ from Figure 6.4. Observe that $C=\{a, b, c, d\}$. We can choose $\{c, d\}$ from $C$ and add $\{e\}$ to obtain $\{c, d, e\}$ which is clearly a circuit hence this example is T-chordal. Now if we let $C_{1}=\{a, b, c, e\}$ and $C_{2}=\{a, b, d, e\}$ which are both circuits then we see that $C_{1} \triangle C_{2}=\{c, d\} \neq C$. This is true for all choices of $C$ and $C_{1}, C_{2}$. Thus this example is not $\mathrm{C}$-chordal.

Finally, Bonin chordality does not imply C-chordality for general matroids as the following counter-example shows.

Example 6.4.10. Consider the rank-4 whirl (see Figure 6.5). This rank 4 matroid with 8 elements has 16 circuits - 12 of which are larger than 3 . For every circuit $C$ of $B_{4,8},|C|>3$, there exist two subsets, $C_{1}, C_{2}$ and an element $e$ outside of $C$ such $C_{1} \cup\{e\}, C_{2} \cup\{e\}$ are circuits and are smaller than $C$. However there is at least one circuit $\left\{b_{1}, b_{2}, b_{4}, d_{1}, d_{2}\right\}$ which can not be partitioned so that if we join the partitions with an element $e \notin C$ both sets are circuits of $B_{4,8}$.

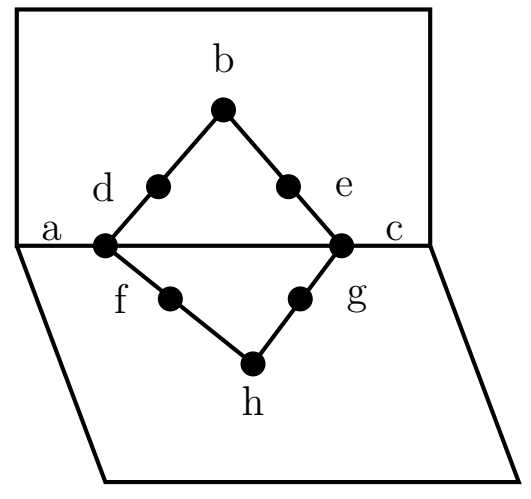

Figure 6.5: The rank 4 matroid with 8 elements. This is of course the rank-4 whirl. 


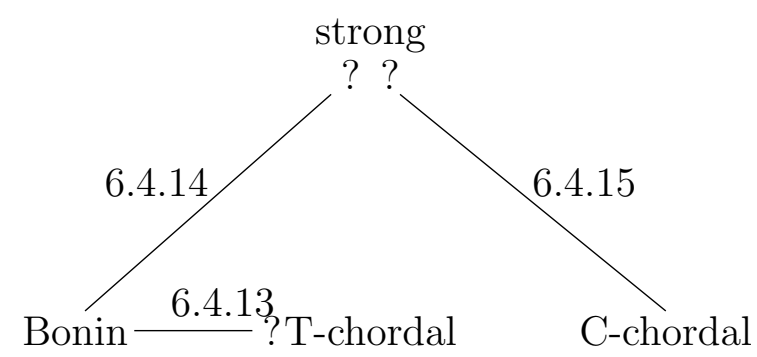

weak

Figure 6.6: How the different open problems relate. $A-? B$ means that we do not know if $A$ implies $B$. The edge labels refer to the open problems that describe each case.

\subsubsection{Open Problems}

As we have seen, we know quite a lot about how our five types of chordality for matroids interrelate for general matroids. First, strong chordality implies Bonin chordality, T-chordality and C-chordality which in turn imply weak chordality for general matroids. We have further seen that C-chordality implies Bonin chordality and T-chordality for general matroids. T-chordality does not imply Bonin chordality nor C-chordality and Bonin chordality does not imply C-chordality. This leaves us with questions about Bonin chordality, T-chordality and C-chordality. See the accompanying digraph in Figure 6.6. First, we ask how do Bonin chordality and T-chordality relate for general matroids? Well we do know two things.

Lemma 6.4.11. Let $M$ be a simple matroid such that every circuit has size at most 5. Then $M$ is T-chordal.

Proof. Let $C$ be a circuit of $M$. If $|C| \leq 4$ we are done. So assume $|C|=5$. Because $M$ is Bonin chordal we know that there are subsets $C_{1}, C_{2} \subseteq C$ and an element $f \notin C$ such that $C_{1} \cup\{f\}$ and $C_{2} \cup\{f\}$ are circuits and $\left|C_{1} \cup\{f\}\right|,\left|C_{2} \cup\{f\}\right|<|C|$. Now if either $\left|C_{1} \cup\{f\}\right|=3$ or $\left|C_{2} \cup\{f\}\right|=3$ 
then we are done. So assume that both $\left|C_{1} \cup\{f\}\right|=4$ and $\left|C_{2} \cup\{f\}\right|=4$. Let $C^{\prime}=C_{1} \cup\{f\}$.

Now by Bonin chordality the circuit $C^{\prime}$ has two subsets $C_{1}^{\prime}, C_{2}^{\prime}$ with an element $g$ such that $C_{1}^{\prime} \cup\{g\}$ and $C_{2}^{\prime} \cup\{g\}$ are triangles. Because two distinct triangles, whose union has rank three, can only intersect in one element, we know that $C_{1}^{\prime} \cap C_{2}^{\prime}=\emptyset$ and so $C_{1}^{\prime}, C_{2}^{\prime}$ forms a partition of $C^{\prime}$. This means that one of $C_{1}^{\prime}$ or $C_{2}^{\prime}$ consists of two elements $\left\{c_{1}, c_{2}\right\}$ of $C$ and $\left\{c_{1}, c_{2}, g\right\}$ is a triangle.

This is true for every circuit of size 5 or less. Thus $M$ is T-chordal.

Corollary 6.4.12. Let $M$ be a simple matroid. Let $r(M) \leq 4$. If $M$ is Bonin chordal then $M$ is T-chordal.

Proof. If $r(M) \leq 4$ then for no circuit $C$ of $M$ can it be that $|C|>5$. Thus Lemma 6.4.11 applies.

This leads to the following open question.

Open Problem 6.4.13. Let $M$ be a matroid. If $M$ is Bonin chordal and $r(M) \geq 5$ is $M$ then T-chordal?

We have investigated this. If there is a counter-example it is certainly a matroid of rank higher than 4 . We have considered possible counter-examples which have ranks of 5 and higher one of which has a circuit of size 6 . Unfortunately, these did not work out when analyzed. Usually in order for the matroid to have a circuit which is not T-chordal while the matroid is Bonin chordal we had to add extra elements which would counteract the properties we were after. Proving the proposition was just as hard. We needed to establish an inductive step which is not clearly obvious to us at this point. 
Open Problem 6.4.14. Let $M$ be a matroid. If $M$ is Bonin chordal then is $M$ strongly chordal?

If we can answer the previous question then this question should be answered. For a Bonin chordal matroid $M$, if $M$ is T-chordal then it may well be strongly chordal. If there is however a counterexample to Open Problem 6.4 .13 then being Bonin chordal can not possibly imply strong chordality. Finally, we have the following question.

Open Problem 6.4.15. Let $M$ be a matroid. If $M$ is $C$-chordal then is $M$ strongly chordal?

We do not know the answer to this question at this time. As we saw earlier if $M$ is $\mathrm{C}$-chordal then $M$ is T-chordal. If $M$ is $\mathrm{C}$-chordal then for any circuit $C$ of size greater than 3 then we can partition $C$ and add an element not in $C$ to achieve two further circuits. The fact of this partition might mean that because there is always at least one triangle formed by a subset $\left\{c_{1}, c_{2}\right\}$ of $C$ and an element $e$ then $\left(C-\left\{c_{1}, c_{2}\right\}\right) \cup e$ is also a circuit. But this is not clear. There may be a clear and higher rank counter-example. 


\section{Chapter 7}

\section{Supersolvability}

We have seen that there are multiple distinct concepts of chordality for general matroids though for binary matroids and graphs these become equivalent. So for matroids in general it is unclear which definition of chordality we would want to use. Moreover, it does not seem that even strong chordality gives us the tree-like matroid structure we desire.

However, there is a related concept to chordality in matroids called supersolvability. In this chapter we consider supersolvability in matroids. First, we examine some previous known results. Sections 7.2, 7.3 and 7.4 are new work though some of the results in section 7.3 rest on previous results. First, we consider how chordality in graphs relates to supersolvability in their cycle matroids.

\subsection{Supersolvablity in Matroids}

Let $M$ be a matroid. A chain of flats of $M$ is a sequence $F_{0}, F_{1}, \ldots, F_{r}$ of flats such that $F_{0} \subsetneq F_{1} \subsetneq \ldots \subsetneq F_{r}$ where $r_{M}\left(F_{i}\right)=i$ for $0 \leq i \leq r$. A chain is a maximal chain if $r=r(M)$. The matroid $M$ is supersolvable if $M$ contains a maximal chain of modular flats. 
Stanley [47, 1972] introduced the concept of supersolvability for lattices but he does discuss supersolvability in the context of graphs and their cycle matroids. He shows how supersolvable cycle matroids are equivalent to chordal graphs as we will see below.

First, Ziegler [49] provides an alternate recursive expression of what it is for a matroid $M$ to be supersolvable.

Definition 7.1.1. A matroid $M$ is recursively supersolvable if either

i. it has $r(M) \leq 2$; or

ii. it has $r(M)>2$ and a modular hyperplane $H \subseteq E(M)$ such that $M \mid H$ is supersolvable.

Lemma 7.1.2. Let $M$ be a matroid. $M$ is supersolvable if and only if it is recursively supersolvable.

Proof. Suppose $M$ is recursively supersolvable. We can show that a maximal chain of modular flats must exist by construction using this recursive relationship. If the matroid is of rank less than or equal to 2 then by default it has a maximal chain of modular flats.

So assume that the matroid has rank greater than 2. Then we know it has a modular hyperplane $H$ such that $M \mid H$ is supersolvable. By following the recursion, and by applying Lemma 4.1.6, we see that $M$ must have a maximal chain of modular flats.

On the other hand suppose that $M$ has a maximal chain of modular flats. We proceed by induction on $r=r(M)$. We can see that if $r \leq 2$, the chain 
consists of a line that is a flat with elements as its hyperplanes. Let $H$ be one of the modular hyperplanes that we have assumed exist. But $M$ must have a modular hyperplane $H$ because it is supersolvable and the matroid $M \mid H$ by definition will have rank $r-1$. Thus if $M$ is supersolvable it must be recursively supersolvable.

\subsubsection{Supersolvability in the Cycle Matroids of Graphs}

The following theorem paraphrases results of Dirac [18] and Stanley [47] for which we provide a proof. The theorem shows the equivalency of supersolvable cycle matroids and their chordal graphs. First, we give a definition. A simplicial ordering of the vertices of a graph $V(G)$ is an ordering $v_{0}, \ldots, v_{n}$ of the vertices of $V(G)$ so that $\mathcal{N}_{G}\left(v_{i}\right)$ is a clique in each induced subgraph $G\left[v_{0}, \ldots, v_{i}\right]$.

Theorem 7.1.3. Given a graph $G$ with cycle matroid $M(G)$ the following are equivalent:

i. $G$ is chordal.

ii. $V(G)$ has a simplicial ordering.

iii. $M(G)$ is supersolvable.

Proof. It is a well known result that $G$ is chordal if and only if it has a simplicial ordering. This follows directly from Theorem 5.1.12. Also see Corollary 9.22 of Bondy and Murty (2008) [9].

Now assume that $G$ has a simplicial ordering $\left(v_{0}, \ldots, v_{n}\right)$. We will prove that $M(G)$ is recursively supersolvable. We do this by proving that $M\left(G\left[v_{0}, \ldots, v_{i-1}\right]\right)$ is a modular hyperplane of $M\left(G\left[v_{0}, \ldots, v_{i}\right]\right)$ for all $i \in[1, \ldots, n]$. 
Because $\left(v_{0}, \ldots, v_{n}\right)$ is a simplicial ordering, $v_{i}$ is a simplicial vertex of $G\left[v_{0}, \ldots, v_{i}\right]$. Let $C^{*}$ be the set of edges incident with $v_{i}$. Then consider $H=E\left(G\left[v_{0}, \ldots, v_{i}\right]\right)-$ $C^{*}$. By Lemma 5.1.2 if $G\left[v_{0}, \ldots, v_{i}\right]$ has exactly one component then $G\left[v_{0}, \ldots, v_{i-1}\right]$ has exactly one component. Therefore the rank of $H$ is $r\left(M\left(G\left[v_{0}, \ldots, v_{i-1}\right]\right)\right)=$ $r\left(M\left(G\left[v_{0}, \ldots, v_{i}\right]-\left\{v_{i}\right\}\right)\right)=r\left(M\left(G\left[v_{0}, \ldots, v_{i}\right]\right)\right)-1$. So $H$ is a hyperplane of $G\left[v_{0}, \ldots, v_{i}\right]$. This means $C^{*}$ is a cocircuit of $G\left[v_{0}, \ldots, v_{i}\right]$.

Now say $e$ and $f$ are elements of $C^{*}$. Then $e$ and $f$ are incident with $v_{i}$. Let $e=\left\{u, v_{i}\right\}$ and $f=\left\{v, v_{i}\right\}$. As $v_{i}$ is a simplicial vertex of $G\left[v_{0}, \ldots, v_{i}\right]$, there is an edge $g=\{u, v\}$. So $\{e, f, g\}$ is a triangle in $G\left[v_{0}, \ldots, v_{i}\right]$. Therefore $g \in \operatorname{cl}_{M\left(G\left[v_{0}, \ldots, v_{i}\right]\right)}(\{e, f\})$. But $g \in H$. By Lemma 4.1.3 $H$ is modular in $M\left(G\left[v_{0}, \ldots, v_{i}\right]\right)$. This is true for any $i \in[1, \ldots, n]$. Thus $M(G)$ is recursively supersolvable. In turn by Lemma 7.1 .2 we see that $M(G)$ is supersolvable.

Next, by Lemma 7.1.2, if $M(G)$ is supersolvable then it is recursively supersolvable. Assume $r(M(G))>2$ otherwise the result is trivial. Then $M(G)$ has a modular hyperplane $H \subseteq E(M(G))$ such that $M(G) \mid H$ is supersolvable of rank $r(M(G))-1$. Now by Lemma 5.2.5 it follows that $E(M(G))-H$ in $G$ is incident with a simplicial vertex in $G$. If we apply this recursively until $r(M) \leq 2$ then we obtain a simplicial ordering of $V(G)$.

We now discuss how supersolvability and chordality in matroids relate both when the matroids are binary and in general. We see that there are still problems that are addressed by including the further requirement of saturation introduced later.

\subsubsection{Supersolvability for Binary Matroids}

First we observe a result of Ziegler [49].

Theorem 7.1.4 (Ziegler 1991 [49]). Let $\mathcal{M}$ be the class of binary matroids that do not contain the Fano plane as a submatroid. Let $M$ be a supersolvable binary matroid. If $M \in \mathcal{M}$ then it is graphic. 
Next Cordovil et al [14] explicitly address the question as to whether binary supersolvable matroids are chordal. Recall the definition of C-chordal for matroids given earlier in Chapter 6 .

Theorem 7.1.5 (Cordovil et al. (2004) [14]). Let $M$ be a simple matroid. If $M$ is binary and supersolvable then $M$ is $C$-chordal.

Next, we show the corollary that, for binary matroids, supersolvability implies chordality in the multiple forms introduced earlier.

Corollary 7.1.6. Let $M$ be a binary matroid. If $M$ is supersolvable then the following are true:

i) $M$ is weakly chordal.

ii) $M$ is Bonin chordal.

iii) $M$ is T-chordal.

iv) $M$ is C-chordal.

v) $M$ is strongly chordal.

Proof. By the theorem of Cordovil et al. [14] (Theorem 7.1.5) if $M$ is binary and supersolvable it is C-chordal. By Theorem 6.3.5 if $M$ is binary and Cchordal then it is also weakly chordal, T-chordal, Bonin chordal and strongly chordal. 


\subsubsection{Supersolvability and Chordality for General Ma- troids}

We now consider a few results comparing supersolvability with chordality for general matroids.

Lemma 7.1.7. Let $M$ be a simple matroid. If $M$ is supersolvable it is $T$ chordal.

Proof. Let $\emptyset \subsetneq F_{1} \subsetneq \ldots \subsetneq F_{r-1} \subsetneq E(M)$ be a maximal chain of modular flats of $M$. Let $C$ be a circuit of $E(M)$. If $C \subseteq F_{r-1}$, then we win by induction by considering the supersolvable matroid $M \mid F_{r-1}$. Now assume that $C-F_{r-1} \neq \emptyset$. If there are at least two elements in $C-F_{r-1}$, let these be $\{a, b\}$. Because $F_{r-1}$ is modular there is an element $c \in F_{r-1}$ such that $\operatorname{cl}_{M}(\{a, b\}) \supseteq c$ and so $\{a, b, c\}$ is a triangle, so we have nothing left to prove. If there is only one element in $C-F_{r-1}$ then $F_{r-1}$ can not be a flat which is a contradiction.

Corollary 7.1.8. Let $M$ be a simple matroid. If $M$ is supersolvable it is weakly chordal.

Proof. We can see this result by first applying Lemma 7.1.7 then applying Lemma 6.2.2,

Now consider the following counter-example which shows that a supersolvable matroid is not necessarily strongly chordal.

Example 7.1.9. Consider the example rank 3, 10 element, matroid $B_{3,10}$ in Figure 7.1. This has at least one maximal chain of modular flats so is supersolvable. For example $\emptyset \subsetneq\left\{p_{1}\right\} \subsetneq\left\{p_{1}, p_{2}, p_{3}, p_{4}, p_{5}, p_{6}\right\} \subsetneq E\left(B_{3,10}\right)$ is a maximal chain of modular flats of $B_{3,10}$. 


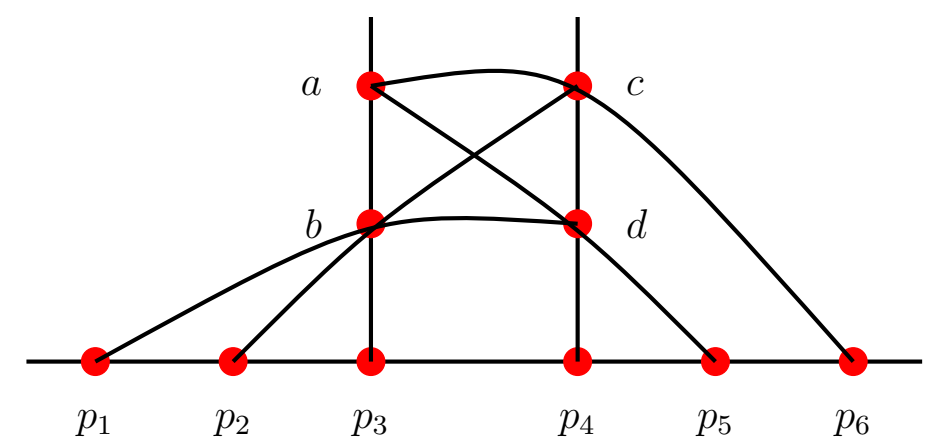

Figure 7.1: $B_{3,10}$ : A counter-example to supersolvable matroids being strongly chordal

However, now consider the circuit $C=\{a, b, c, d\}$. Then consider any two element subset of $C$ such as $\{a, d\}$. There is an element $p_{5}$ not in $C$ such that there is a triangle $\left\{a, d, p_{5}\right\}$. But $(C-\{a, d\}) \cup\left\{p_{5}\right\}=\left\{b, c, p_{5}\right\}$ is not a circuit. This is clearly true for all other two element subsets of $C$. As a result, $B_{3,10}$ is not strongly chordal.

This means that we will need to explicitly require that our matroids be supersolvable instead of just stating that they are strongly chordal.

\subsection{Supersolvable Saturation}

As we saw earlier, if a graph is chordal then every minimal vertex separation is a clique cut. Let $M$ be a matroid. As we shall see, the matroidal analogue of a clique cut is to have a separation $(A, B)$ in $M$ such that $\operatorname{cl}(A) \cap \operatorname{cl}(B)$ is a modular flat of $M$. Now recall that, for a flat $F$ in $M$, if $M \mid F$ has no vertical separations then $F$ is round. In turn $M$ is saturated if every round flat of $M$ is modular. Note that saturate in this context is different from the meaning of saturated in the context of "saturated chain". We can ask the question as to whether a supersolvable matroid is necessarily saturated. This turns out to be false. Consider the following counter-example. 


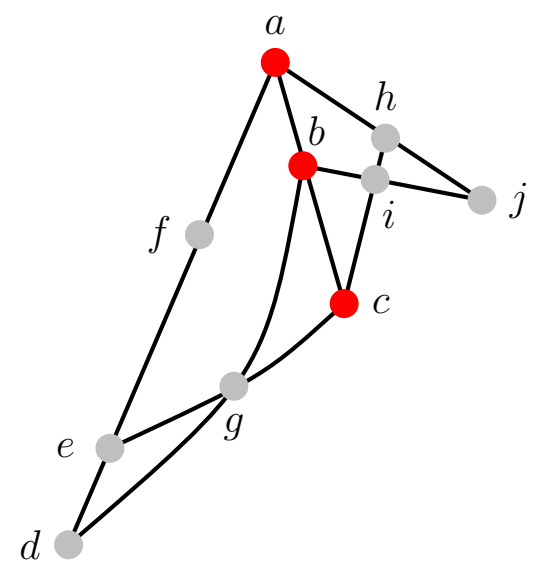

Figure 7.2: A rank 4 matroid that shows that supersolvability does not imply saturation

Example 7.2.1. Observe that the rank 4 matroid $M$ in Figure 7.2 is certainly supersolvable. One maximal chain of modular flats would be $\emptyset \subsetneq$ $\{d\} \subsetneq\{d, e, f, a\} \subsetneq\{d, e, f, a, b, c\} \subseteq E(M)$. However, consider the flat $F=\{a, b, c\}$. Now $F$ is round since no separation of $F$ is vertical. However, the flat is not modular. Consider the flat $Z=\{f, g\}$. Then $r(F)=2$ and $r(Z)=2$ but $r(F \cup Z)=r(\{a, b, c, f, g\})=3$ while $r(F \cap Z)=r(\emptyset)=0$ and so $r(F)+r(Z)>r(F \cup Z)+r(F \cap Z)$.

Furthermore, consider a minimal vertical $k$-separation $(A, B)$ of a supersolvable matroid $M$. We are particularly interested in the flat that forms the intersection $\operatorname{cl}(A) \cap \operatorname{cl}(B)$. For example, in the counter-example in Figure 7.2. we can let $A=\{a, b, c, d, e, f, g\}, B=\{a, b, c, h, i, j\}$ and $\operatorname{cl}(A) \cap \operatorname{cl}(B)=$ $\{a, b, c\}$. We saw above that $\{a, b, c\}$ is not modular.

Thus the example in Figure 7.2 shows us that supersolvability is not enough to guarantee that $\operatorname{cl}(A) \cap \operatorname{cl}(B)$ is modular. This leads us to the following definition. Let $M$ be a matroid. Recall that $M$ is saturated if every round flat $F$ of $M$ is modular. Remember that a flat $F$ is round if $M \mid F$ is round. Now we define $M$ to be supersolvably saturated if $M$ is both supersolvable and saturated.

We now give some useful results concerning supersolvably saturated ma- 
troids. First, we can restrict to modular flats and retain the property of being supersolvably saturated. We can denote the maximal chain of flats $F_{0} \subsetneq F_{1} \subsetneq F_{2} \subsetneq \ldots \subsetneq F_{r}$ by $\left(F_{0}, F_{1}, F_{2}, \ldots, F_{r}\right)$.

Lemma 7.2.2. Let $G$ be a modular flat of the rank $r$ matroid $M$. Let $\left(F_{0}, F_{1}, F_{2}, \ldots, F_{r}\right)$ be a maximal chain of flats. Then $\left(F_{0} \cap G, F_{1} \cap G, F_{2} \cap\right.$ $\left.G, \ldots, F_{r} \cap G\right)$ contains a maximal chain of flats of $M \mid G$.

Proof. Let $d=r-r(G)$ so that $r(G)=r-d$ for $d \in\{1, \ldots, r\}$. Also, remember that because $\left(F_{0}, F_{1}, \ldots, F_{r}\right)$ is a maximal chain of flats, we know that $r\left(F_{i+1}\right)-r\left(F_{i}\right)=1$. We first establish a claim.

7.2.2.1. $r\left(F_{i+1} \cap G\right)-r\left(F_{i} \cap G\right) \leq 1$ for $i \in\{0,1, \ldots, r\}$.

Proof. Let $i \in\{0, \ldots, r\}$. Consider $F_{i}$. If $F_{i} \subseteq G$, then $F_{i} \cap G=F_{i}$ so $r\left(F_{i} \cap G\right)=r\left(F_{i}\right)$. Say $F_{i} \nsubseteq G$. Then we can see that $r-d \leq r\left(F_{i} \cup G\right) \leq$ $2 r-d$. This means we have $r\left(F_{i}\right)+(r-d)-(2 r-d) \leq r\left(F_{i}\right)+r(G)-r\left(F_{i} \cup G\right) \leq$ $r\left(F_{i}\right)+(r-d)-(r-d)$. In turn, because $G$ is modular, we have that $r\left(F_{i}\right)-r \leq r\left(F_{i} \cap G\right) \leq r\left(F_{i}\right)$.

Now let $i \in\{0, \ldots r-1\}$ and consider $F_{i}$ and $F_{i+1}$. If $F_{i+1} \subseteq G$, then $F_{i} \subseteq G$, so $r\left(F_{i+1} \cap G\right)-r\left(F_{i} \cap G\right)=r\left(F_{i+1}\right)-r\left(F_{i}\right)=1$. If $F_{i+1} \nsubseteq G$, then we have two subcases.

i) If $F_{i} \not \subseteq G$ then we have

$$
\begin{aligned}
& 1 \\
= & \left(r\left(F_{i+1}\right)-r\right)-\left(r\left(F_{i}\right)-r\right) \\
\leq & r\left(F_{i+1} \cap G\right)-r\left(F_{i} \cap G\right) \\
\leq & r\left(F_{i+1}\right)-r\left(F_{i}\right) \\
= & 1
\end{aligned}
$$


Thus, $r\left(F_{i+1} \cap G\right)-r\left(F_{i} \cap G\right)=1$.

ii) Now consider the case where $F_{i} \subseteq G$. Let $B$ be a basis of $M \mid F_{i}$ (observing that now $\left.F_{i}=F_{i} \cap G\right)$. Let $B^{\prime}$ be a basis of $M \mid\left(F_{i+1} \cap G\right)$ such that $B \subseteq B^{\prime}$. Now assume that there are at least two distinct elements $\{x, y\}$ in $B^{\prime}-B$. Then $B^{\prime}$ is independent and $B^{\prime} \subseteq F_{i+1}$ so $r\left(F_{i+1}\right) \geq\left|B^{\prime}\right| \geq|B|+2=r\left(F_{i}\right)+2$. But this contradicts the fact that $r\left(F_{i+1}\right)-r\left(F_{i}\right)=1$. Thus, it must be that $r\left(F_{i+1} \cap G\right)-r\left(F_{i} \cap G\right) \leq 1$.

In both cases $r\left(F_{i+1} \cap G\right)-r\left(F_{i} \cap G\right) \leq 1$.

Next observe that $r\left(F_{r} \cap G\right)=r-d$ and $r\left(F_{0} \cap G\right)=0$ for $d \in\{1, \ldots, r\}$. Let $J \subseteq\{1, \ldots, r\}$ be maximal such that, for all $j \in J, r\left(F_{j+1}\right)-r\left(F_{j}\right)=1$. If $1 \leq j \leq r-d$ then $\left(F_{0} \cap G, F_{1} \cap G, \ldots, F_{r-d} \cap G\right)$ is a maximal chain of flats of $M \mid G$. That is, there is a greatest $i$ with $F_{i} \subseteq G$, so one term in the sequence of intersections is repeated. When this repetition is eliminated, we have a maximal chain of flats in $M \mid G$.

Next, we consider when a restriction $M \mid F$ of a supersolvably saturated matroid $M$ is supersolvable and indeed saturated.

Corollary 7.2.3. Let $G$ be a modular flat of the supersolvable matroid $M$. Then $M \mid G$ is supersolvable.

Proof. Let $\left(F_{0}, \ldots, F_{r}\right)$ be a maximal chain of modular flats of $M$. By Lemma $7.2 .2\left(F_{0} \cap G, \ldots, F_{r} \cap G\right)$ contains a maximal chain of flats. Then by Lemma 4.1.7, for each $i \in\{0, \ldots, r\}, F_{i} \cap G$ is modular. Thus $M \mid G$ is supersolvable.

Lemma 7.2.4. Let $F$ be a flat of the saturated matroid $M$. Then $M \mid F$ is saturated. 
Proof. Let $G$ be a round flat of $M \mid F$. Then, as $F$ is a flat of $M$, the set $G$ is round in $M$. Hence $G$ is a modular flat in $M$. By Lemma 4.1.5 we see that $G$ is modular in $M \mid F$.

Corollary 7.2.5. If $M$ is a supersolvably saturated matroid and $G$ is a modular flat of $M$ then $M \mid G$ is supersolvably saturated.

Proof. By Corollary 7.2 .3 since $M$ is supersolvable then $M \mid G$ is supersolvable. $G$ is a flat of $M$ and $M$ is saturated so by Lemma 7.2.4 we see that $M \mid G$ is saturated.

Note that for this corollary, if $G$ is a hyperplane, we do not need that $G$ be modular although the argument presented here used that fact. We could instead have taken account of the fact that there is a maximal chain of modular flats.

Now consider a modular hyperplane $H$ of a simple matroid $M$ with $E-H=$ $\left\{p_{1}, \ldots, p_{n}\right\}$. For distinct $i, j \in\{1, \ldots, n\}$ we denote by $p_{i j}$ the element $\operatorname{cl}\left(\left\{p_{i}, p_{j}\right\}\right) \cap H$. Then we have the following result.

Lemma 7.2.6. Let $M$ be a simple matroid and $H$ be a modular hyperplane in $M$. Let $p_{i}, p_{j}, p_{k}$ for $(1 \leq i<j<k<|E(M)-H|)$ be independent in $E(M)-H$. Then $r_{M}\left(\left\{p_{i j}, p_{j k}, p_{i k}\right\}\right)=2$.

Proof. Observe that $r_{M}\left(\operatorname{cl}_{M}\left(\left\{p_{i}, p_{j}, p_{k}\right\}\right) \cap H\right) \leq r_{M}\left(\left\{p_{i}, p_{j}, p_{k}\right\}\right)+r_{M}(H)-$ $r(M)=3+(r-1)-r=2$. But by definition $\left\{p_{i j}, p_{j k}, p_{i k}\right\} \subseteq \operatorname{cl}_{M}\left(\left\{p_{i}, p_{j}, p_{k}\right\}\right) \cap$ $H$. Hence $r_{M}\left(\left\{p_{i j}, p_{j k}, p_{i k}\right\}\right) \leq 2$.

Assume that $p_{i j}=p_{i k}$. Then $\left\{p_{i j}, p_{i}, p_{j}\right\}$ is a triangle as is $\left\{p_{i j}, p_{i}, p_{k}\right\}$. This means that $p_{j}$ and $p_{k}$ are both in the closure of $\left\{p_{i j}, p_{1}\right\}$. This means that $r\left(\left\{p_{i j}, p_{i}, p_{j}, p_{k}\right\}\right)=2$. But this contradicts the fact that $r\left(\left\{p_{i}, p_{j}, p_{k}\right\}\right)=3$. Thus $p_{i j}, p_{j k}, p_{i k}$ must all be distinct.

As $M$ is simple $r_{M}\left(\left\{p_{i j}, p_{j k}, p_{i k}\right\}\right) \geq 2$. Therefore $r_{M}\left(\left\{p_{i j}, p_{j k}, p_{i k}\right\}\right)=2$. 
If we let $C=E(M)-H$ then we denote $L(C)=\bigcup_{\{a, b\} \subseteq C} \operatorname{cl}_{M}(\{a, b\}) \cap H$. Note that for later reference we use the language of separations but we only need $(X, Y)$ to be a partition of $H$.

Lemma 7.2.7. Let $H$ be a modular hyperplane in a matroid $M$. Let $C=$ $E(M)-H$ and let $(X, Y)$ be a partition of $H$ with $\lambda_{M \mid H}(X, Y)=k$. Then the following properties hold.

(i) Either $L(C) \subseteq \mathrm{cl}_{M}(X)$ or $L(C) \subseteq \mathrm{cl}_{M}(Y)$.

(ii) If $L(C) \subseteq \operatorname{cl}_{M}(X)$ then $r_{M}(X \cup C)=r_{M}(X)+1$.

(iii) If $L(C) \subseteq \operatorname{cl}_{M}(X)$ then $\lambda_{M}(X \cup C, Y)=\lambda_{M \mid H}(X, Y)=k$.

Proof. First we prove that assertion (i) is satisfied. Let $P=\left\{p_{1}, p_{2}, \ldots, p_{n}\right\}$ be a basis of $C . L(C)$ is defined as denoted above. Observe that $\mathrm{cl}_{M}(P)=$ $\operatorname{cl}_{M}(C)$. Assume for a contradiction that there exist $i, j, k, l$ such that $p_{i j} \in$ $X-\mathrm{cl}_{M}(Y)$ and $p_{k l} \in Y-\operatorname{cl}_{M}(X)$. We first observe the following.

7.2.7.1. For indices $r, s, t$ either $\left\{p_{r s}, p_{s t}, p_{r t}\right\} \subseteq \operatorname{cl}_{M}(X)$ or $\left\{p_{r s}, p_{s t}, p_{r t}\right\} \subseteq$ $\mathrm{cl}_{M}(Y)$.

Proof. By Lemma 7.2.6 the points $\left\{p_{r s}, p_{s t}, p_{r t}\right\}$ form a circuit of size 3. Every element of this circuit is in the closure of the other two. Thus, without loss of generality, if $p_{r s} \in \operatorname{cl}_{M}(X)$ and $p_{s t} \in \operatorname{cl}_{M}(X)$ then $p_{r t} \in \operatorname{cl}_{M}\left(\left\{p_{r s}, p_{s t}\right\}\right) \subseteq$ $\mathrm{cl}_{M}(X)$.

The claim clearly holds where each $p_{i j}$ is in $\operatorname{cl}_{M}(X) \cap \mathrm{cl}_{M}(Y)$. So now assume that there is some $i, j$ with $p_{i j} \in Y-\operatorname{cl}_{M}(X)$. Consider indices $s, t$ with $\{i, j\} \cap\{s, t\}=\emptyset$. Applying step 7.2.7.1 to $\left\{p_{i}, p_{j}, p_{s}\right\}$ gives $\left\{p_{i s}, p_{j s}\right\} \subseteq$ $\operatorname{cl}_{M}(Y)$. Likewise, $\left\{p_{i t}, p_{j t}\right\} \subseteq \operatorname{cl}_{M}(Y)$. Also $p_{s t} \in \operatorname{cl}_{M}(Y)$ since $\left\{p_{i s}, p_{i t}\right\} \subseteq$ 
$\mathrm{cl}_{M}(Y)$. Assertion (i) now follows.

Now consider assertion (ii). Since $X$ is contained in the hyperplane whose complement is $C$, we have $r_{M}(X \cup C)>r_{M}(X)$. In order to prove that $r_{M}(X \cup C)=r_{M}(X)+1$, it suffices to show that for any $p_{i}$ in the basis $P$ of $C$, we have $P \subseteq \operatorname{cl}_{M}\left(X \cup p_{i}\right)$. This holds since each $p_{j} \in P-p_{i}$ is in the 3 -circuit with $p_{i}$ and the element $p_{i j} \in \mathrm{cl}_{M}(X)$.

Finally consider assertion (iii). By the definition of the connectivity function we know that

$$
\begin{aligned}
& \lambda_{M}(X \cup C, Y) \\
= & r_{M}(X \cup C)+r_{M}(Y)-r(M) \\
= & r_{M}(X)+1+r_{M}(Y)-r(M) \\
= & r_{M}(X)+r_{M}(Y)-(r(M)-1) \\
= & r_{M \mid H}(X)+r_{M \mid H}(Y)-r_{M \mid H}(H) \\
= & \lambda_{M \mid H}(X, Y)=k .
\end{aligned}
$$

Corollary 7.2.8. Let $H$ be a modular hyperplane of the matroid $M$ and let $C^{*}=E(M)-H$. Let $(X, Y)$ be a vertical $k$-separation of $M \mid H$. Then the following hold.

(i) Either $\operatorname{cl}_{M}\left(C^{*}\right) \cap H \subseteq \operatorname{cl}(X)$ or $\operatorname{cl}_{M}\left(C^{*}\right) \cap H \subseteq \operatorname{cl}_{M}(Y)$.

(ii) If $\mathrm{cl}_{M}\left(C^{*}\right) \cap H \subseteq \mathrm{cl}_{M}(X)$, then $\left(X \cup C^{*}, Y\right)$ is a vertical $k$-separation of $M$.

Next we introduce the concept of modular separation (see below for the definition). First, we must discuss separations in general. 


\subsection{Bridges and Minimal Separations}

As we have seen earlier, a minimal vertex cut of a graph is a minimal set of vertices whose deletion increases the number of components of the graph. We have seen that minimal vertex cuts of chordal graphs are clique cuts and this is the primary reason why the reduced clique graph $C_{r}(G)$ of a chordal graph $G$ is highly structured. Our goal is to prove that an analogous property holds for supersolvably saturated matroids.

In matroids we cannot focus on cuts, but cuts in graphs induce $k$-separations and we can focus on these. Let $(X, Y)$ be a vertical $k$-separation in the matroid $M$. Then a bridge of $(X, Y)$ is a component of $M / \mathrm{cl}_{M}(X)$ or $M / \mathrm{cl}_{M}(Y)$.

A partition $(X, Y)$ is a $k$-separation if and only if $\lambda_{M}(X) \leq k-1$.

Lemma 7.3.1. Let $Y$ and $Z$ be disjoint sets in a matroid $M$. Then

$$
\lambda_{M}(Z) \leq \lambda_{M / Y}(Z)+\lambda_{M}(Y)
$$

Proof. Let $W=E(M)-(Z \cup Y)$. Recall from basic matroid theory that $r_{M / Y}(A)=r_{M}(A \cup Y)-r_{M}(Y)$ for all $A \subseteq E$. Using this fact, the submodularity of the rank function and the definition of $\lambda$, we obtain the following.

$$
\begin{aligned}
& \lambda_{M / Y}(Z)+\lambda_{M}(Y) \\
= & r_{M / Y}(Z)+r_{M / Y}(W)-r(M / Y)+r_{M}(Y)+r_{M}(Z \cup W)-r(M) \\
= & r_{M}(Z \cup Y)-r_{M}(Y)+r_{M}(W \cup Y)-r_{M}(Y)-r(M)+r_{M}(Y)+ \\
& \quad r_{M}(Y)+r_{M}(Z \cup W)-r(M) \\
= & r_{M}(Z \cup Y)+r_{M}(Z \cup W)+r_{M}(W \cup Y)-2 r(M) \\
\geq & r_{M}(Z)+r(M)+r_{M}(W \cup Y)-2 r(M) \\
= & r_{M}(Z)+r_{M}(W \cup Y)-r(M) \\
= & \lambda_{M}(Z) .
\end{aligned}
$$


Lemma 7.3.2. Let $Z$ be a bridge of the $k$-separation $(X, Y)$. Then $\lambda_{M}(Z) \leq$ $k-1$.

Proof. Note that either $Z \subseteq X$ or $Z \subseteq Y$. Assume the former holds. Note that $\lambda_{M}\left(\operatorname{cl}_{M}(Y)\right) \leq \lambda_{M}(Y)$. By the definition of bridge $\lambda_{M / c l_{M}(Y)}(Z)=0$. Then by Lemma 7.3.1.

$$
\lambda_{M}(Z) \leq \lambda_{M / \mathrm{cl}_{M}(Y)}(Z)+\lambda_{M}(\operatorname{cl}(Y)) \leq 0+\lambda_{M}(Y) \leq k-1
$$

Let $(X, Y)$ be a vertical $k$-separation of the matroid $M$. Then $(X, Y)$ is minimal if $\lambda_{M}(Z)=k-1$ for every bridge $Z$ of $(X, Y)$.

Lemma 7.3.3. Let $(X, Y)$ be a minimal vertical $k$-separation of the matroid M. Then $r_{M}\left(Y-\operatorname{cl}_{M}(X)\right)=r_{M}(Y)$.

Proof. Assume otherwise. Then $\lambda_{M}\left(Y-\mathrm{cl}_{M}(X), \mathrm{cl}_{M}(X)\right)<k-1$. Let $Z \subseteq Y-\mathrm{cl}_{M}(X)$ be a bridge of $(X, Y)$. Then $Z$ is also a bridge of $(Y-$ $\left.\mathrm{cl}_{M}(X), \mathrm{cl}_{M}(X)\right)$. By Lemma 7.3.2.

$$
\lambda_{M}(Z) \leq \lambda_{M}\left(Y-\mathrm{cl}_{M}(X), \mathrm{cl}_{M}(X)\right)<k-1
$$

This contradicts the assumption that $(X, Y)$ is a minimal separation.

Lemma 7.3.4. Let $(X, Y)$ be a vertical $k$-separation of the matroid $M$. Then the following hold.

(i) If $(X, Y)$ is minimal then $\lambda_{M}(X, Y)=\lambda_{M}\left(\operatorname{cl}_{M}(X), Y-\operatorname{cl}_{M}(X)\right)$.

(ii) If $(X, Y)$ is minimal then the bridges of $(X, Y)$ are equal to the bridges of $\left(\operatorname{cl}_{M}(X), Y-\operatorname{cl}_{M}(X)\right)$.

(iii) The separation $(X, Y)$ is minimal if and only if $\left(\operatorname{cl}_{M}(X), Y-\mathrm{cl}_{M}(X)\right)$ is minimal. 
Proof. Consider property (i). By Lemma 7.3.3 $r_{M}\left(Y-\mathrm{cl}_{M}(X)\right)=r_{M}(Y)$. This means that $\lambda_{M}(X, Y)=r_{M}(X)+r_{M}(Y)-r(M)=r_{M}\left(\operatorname{cl}_{M}(X)\right)+$ $r_{M}\left(Y-\mathrm{cl}_{M}(X)\right)-r(M)=\lambda_{M}\left(\operatorname{cl}_{M}(X), Y-\mathrm{cl}_{M}(X)\right)$.

Now consider property (ii). The bridges of $(X, Y)$ are the components of $M / \mathrm{cl}_{M}(Y)$ and the bridges of $\left(\mathrm{cl}_{M}(X), Y-\mathrm{cl}_{M}(X)\right)$ are the components of $M / \mathrm{cl}_{M}\left(Y-\mathrm{cl}_{M}(X)\right)$. By Lemma 7.3.3 $r_{M}(Y)=r_{M}\left(Y-\mathrm{cl}_{M}(X)\right)$. This means by Oxley [41] that $\operatorname{cl}_{M}(Y)=\mathrm{cl}_{M}\left(Y-\mathrm{cl}_{M}(X)\right)$. But then $M / \mathrm{cl}_{M}(Y)=M / \mathrm{cl}_{M}\left(Y-\mathrm{cl}_{M}(X)\right)$.

Finally consider property (iii). If $\left(\mathrm{cl}_{M}(X), Y-\mathrm{cl}_{M}(X)\right)$ is a minimal vertical separation then for every bridge $Z$ of $\left(\operatorname{cl}_{M}(X), Y-\operatorname{cl}_{M}(X)\right)$ it follows that $\lambda_{M}(Z)=k-1$. But by property (ii) all the bridges of $\left(\operatorname{cl}_{M}(X), Y-\operatorname{cl}_{M}(X)\right)$ are equal to the bridges of $(X, Y)$. Hence $(X, Y)$ must also be a minimal vertical $k$-separation. The same follows in the other direction because of property (ii).

Lemma 7.3.5. Let $H$ be a modular hyperplane of the matroid $M$, let $C^{*}=$ $E(M)-H$, and let $(X, Y)$ be a vertical $k$-separation of $M \mid H$ such that $(X \cup$ $\left.C^{*}, Y\right)$ is a minimal vertical $k$-separation of $M$. Then $(X, Y)$ is a minimal vertical $k$-separation of $M \mid H$.

Proof. Consider the bridges of $\left(X \cup C^{*}, Y\right)$ contained in $X \cup C^{*}$. These are the components of the matroid $M / \mathrm{cl}_{M}(Y)$. By Lemma 4.1.10, $H-\mathrm{cl}_{M}(Y)$ is a modular hyperplane of $M / \mathrm{cl}_{M}(Y)$ and $C^{*}$ is, of course the cocircuit complementary to this hyperplane. It follows from this that, in $M / \mathrm{cl}_{M}(Y)$, if $c, c^{\prime} \in C^{*}$, and $\left\{c, c^{\prime}\right\}$ is independent, then there is a triangle containing $\left\{c, c^{\prime}\right\}$ so that $c$ and $c^{\prime}$ are in the same component of $M / \mathrm{cl}_{M}(Y)$.

One possibility is that $C^{*}$ is a component of $M / \mathrm{cl}_{M}(Y)$.

7.3.5.1. $C^{*}$ is a component of $M / \mathrm{cl}_{M}(Y)$ if and only if $r_{M / \mathrm{cl}_{M}(Y)}\left(C^{*}\right)=1$, that is, if and only if $r_{M}\left(C^{*} \cup Y\right)=r_{M}(Y)+1$. 
Proof. Certainly $r_{M / \mathrm{cl}_{M}(Y)}\left(C^{*}\right)>0$. Say $r_{M / \mathrm{cl}_{M}(Y)}\left(C^{*}\right)=1$. Then in this case, as $H-\operatorname{cl}_{M}(Y)$ is a hyperplane of $M / \mathrm{cl}_{M}(Y)$ we see that $C^{*}$ is a component of $M / \mathrm{cl}_{M}(Y)$ as required.

Assume that $r_{M / \mathrm{cl}_{M}(Y)}\left(C^{*}\right)>1$. Let $\left\{c, c^{\prime}\right\}$ be elements of $C^{*}$ that are independent in $M / \mathrm{cl}_{M}(Y)$. As $H-\mathrm{cl}_{M}(Y)$ is a modular hyperplane of $M / \mathrm{cl}_{M}(Y)$, there is an element $z \in H-\operatorname{cl}_{M}(Y)$ such that $\left\{z, c, c^{\prime}\right\}$ is a triangle in $M / \mathrm{cl}_{M}(Y)$. Thus $z$ is in the same connected component of $M / \mathrm{cl}_{M}(Y)$ as $C^{*}$. Hence $C^{*}$ is not a component of $M / \mathrm{cl}_{M}(Y)$ and the lemma follows.

Let $\left\{Z_{1}, Z_{2}, \ldots, Z_{n}\right\}$ be the components of $(M \mid H) / \mathrm{cl}_{M}(Y)$.

7.3.5.2. If $r_{M / \mathrm{cl}_{M}(Y)}\left(C^{*}\right)>1$, then, for some $i \in\{1,2, \ldots, n\}$, the components of $M / \mathrm{cl}_{M}(Y)$ are $\left\{Z_{1}, Z_{2}, \ldots, Z_{i-1}, Z_{i} \cup C^{*}, Z_{i+1}, \ldots, Z_{n}\right\}$.

Proof. Say $B=\left\{b_{1}, b_{2}, \ldots, b_{s}\right\}$ is a basis of $\left(M / \mathrm{cl}_{M}(Y)\right) \mid C^{*}$. Let $B^{\prime}=\bigcup_{1 \leq i<j \leq n} \operatorname{cl}_{M / \mathrm{cl}_{M}(Y)}\left(\left\{b_{i}, b_{j}\right\}\right) \cap\left(H-\mathrm{cl}_{M}(Y)\right)$. By Lemma 4.2.5. $\left(M / \mathrm{cl}_{M}(Y)\right) \mid B^{\prime}$ is isomorphic to the cycle matroid of a complete graph and is therefore a connected matroid. Thus $B^{\prime} \subseteq Z_{i}$ for some $i \in\{1,2, \ldots, n\}$. But the members of $B$ are in triangles with the members of $B^{\prime}$ and hence are in the same component. Moreover $C^{*} \subseteq \operatorname{cl}_{M / \mathrm{cl}_{M}(Y)}\left(B^{\prime}\right)$. We deduce that $Z_{i} \cup C^{*}$ belong to the same component of $M / \operatorname{cl}_{M}(Y)$. But $r_{M / \mathrm{cl}_{M}(Y)}\left(Z_{i} \cup C^{*}\right)=r_{M / \mathrm{cl}_{M}(Y)}\left(Z_{i}\right)+1$. We therefore have

$$
\begin{aligned}
& r_{M / \mathrm{cl}_{M}(Y)}\left(Z_{1}\right)+\cdots+r_{M / \mathrm{cl}_{M}(Y)}\left(Z_{i-1}\right)+r_{M / \mathrm{cl}_{M}(Y)}\left(Z_{i} \cup C^{*}\right) \\
& \quad+r_{M / \mathrm{cl}_{M}(Y)}\left(Z_{i+1}\right)+\cdots+r_{M / \mathrm{cl}_{M}(Y)}\left(Z_{n}\right) \\
= & r_{M / \mathrm{cl}_{M}(Y)}\left(Z_{1}\right)+r_{M / \mathrm{cl}_{M}(Y)}\left(Z_{2}\right)+\cdots+r_{M / \mathrm{cl}_{M}(Y)}\left(Z_{n}\right)+1 \\
= & r\left(M / \mathrm{cl}_{M}(Y) .\right.
\end{aligned}
$$

Hence $Z_{i} \cup C^{*}$ is a component of $M / \mathrm{cl}_{M}(Y)$. 
We now prove that $(X, Y)$ is a minimal $k$-separation of $M \mid H$. It is easily seen that the bridges of $(X, Y)$ contained in $Y$ are the same as the bridges of $\left(X \cup C^{*}, Y\right)$ contained in $Y$. By Corollary 7.2.8, these bridges have the same connectivity in $M$ and $M \mid H$. Thus, if $W$ is such a bridge

$$
\lambda_{M \mid H}(W)=\lambda_{M}\left(X \cup C^{*}, Y\right)=\lambda_{M \mid H}(X, Y)=k-1 .
$$

Consider the bridges $\left\{Z_{1}, Z_{2}, \ldots, Z_{n}\right\}$ of $M \mid H$ contained in $X$. Say $Z$ is such a bridge. By Claim 7.3.5.2, one possibility is that $Z \cup C^{*}$ is a bridge of $\left(X \cup C^{*}, Y\right)$. Then, by Corollary 7.2.8, $\lambda_{M}\left(Z \cup C^{*}\right)=\lambda_{M \mid H}(Z)$ so that $\lambda_{M \mid H}(Z)=\lambda_{M \mid H}(X, Y)$. Otherwise, by Claims 7.3.5.1 and 7.3.5.2, $Z$ is a bridge of $\left(X \cup C^{*}, Y\right)$. Again, by Corollary 7.2.8, $\lambda_{M \mid H}(Z)=\lambda_{M}(Z)=k-1$.

We deduce that the connectivity of all of the bridges of $(X, Y)$ in $M \mid H$ is equal to the connectivity of $(X, Y)$. This proves that $(X, Y)$ is indeed a minimal separation in $M \mid H$.

We finally provide a useful lemma showing that if $M$ has a vertical $k$-separation then it must have a minimal vertical $k$-separation.

Lemma 7.3.6. Let $M$ be a matroid. Let $k$ be a positive integer. Let $(X, Y)$ be a vertical $k$-separation of $M$. Then $M$ has a minimal vertical $k^{\prime}$-separation where $k^{\prime} \leq k$.

Proof. We proceed by induction on the size of $X$. Suppose we have chosen the $k$-separation $(X, Y)$ so that $X$ is as small as possible and $(X, Y)$ is vertical. If $(X, Y)$ is minimal we are done. Otherwise by hypothesis any smaller $k^{\prime}$-separation is minimal for $k^{\prime} \leq k$.

If $(X, Y)$ is not minimal then for one of the bridges, $Z$, of $(X, Y)$ we have that $\lambda(Z) \leq k^{\prime \prime}<k$. If $(Z, E-Z)$ is a vertical $k^{\prime \prime}$-separation then by 
hypothesis $(Z, E-Z)$ is minimal. If so we are done and we just use the minimal vertical $k^{\prime \prime}$-separation $\left(Z, E_{Z}\right)$.

If $\left(Z, E_{Z}\right)$ is not a vertical $k^{\prime \prime}$-separation then consider the $k$-separation $(X-$ $Z, Y \cup Z)$. This is vertical because $Z \subseteq \operatorname{cl}(Y)$. Because $|X-Z| \leq|X|$ we know by the inductive hypothesis that $(X-Z, Y \cup Z)$ is minimal.

\subsection{Modular Separations}

A $k$-separation $(X, Y)$ in the matroid $M$ is modular if $r\left(\operatorname{cl}_{M}(X) \cap \operatorname{cl}_{M}(Y)\right)=$ $\lambda_{M}(X, Y)$ and $\mathrm{cl}_{M}(X) \cap \mathrm{cl}_{M}(Y)$ is a round modular flat of $M$. We refer to the modular $k$-separation as a modular separation. Given a $k$-separation $(A, B)$ of $M$, we call the guts of this $k$-separation to be $\operatorname{cl}_{M}(A) \cap \mathrm{cl}_{M}(B)$.

For chordal graphs we know that every minimal vertex cut $X$ is a clique cut in that $G[X]$ is a clique. The analogous result that we shall prove for supersolvably saturated matroids is that every minimal vertical $k$-separation is a modular separation. We work towards this goal now. The following corollary restates an important result in slightly different terms.

Corollary 7.4.1. Let $H$ be a modular hyperplane of the matroid $M$ and let $C^{*}=E(M)-H$. If $r_{M}\left(C^{*}\right)=s$, then $M \mid\left(\operatorname{cl}\left(C^{*}\right) \cap H\right)$ has a restriction isomorphic to $M\left(K_{s}\right)$.

Proof. This follows immediately from Lemma 4.2.5.

Lemma 7.4.2. Let $H$ be a modular hyperplane of the supersolvably saturated matroid $M$ and let $C^{*}=E(M)-H$. Then $\left(C^{*}, H\right)$ is a modular separation.

Proof. Say $r_{M}\left(C^{*}\right)=s$. By Lemma 7.4.1, $\mathrm{cl}_{M}\left(C^{*}\right) \cap H$ contains a restriction isomorphic to $M\left(K_{s}\right)$. Now

$$
r_{M}\left(H \cap \mathrm{cl}_{M}\left(C^{*}\right)\right)=r_{M}(H)+s-\left(r_{M}(H)+1\right)=s-1=r\left(M\left(K_{s}\right)\right) .
$$


Thus our copy of $M\left(K_{s}\right)$ spans $H \cap \mathrm{cl}_{M}\left(C^{*}\right)$. Also $M\left(K_{s}\right)$ is round. As $H \cap \mathrm{cl}_{M}\left(C^{*}\right)$ contains a round spanning subset we deduce that $H \cap \mathrm{cl}_{M}\left(C^{*}\right)$ is also round. By the definition of supersolvably saturated matroid we see that $H \cap \operatorname{cl}_{M}\left(C^{*}\right)$ is modular. Hence $\left(C^{*}, H\right)$ is a modular separation.

Lemma 7.4.3. Let $(X, Y)$ be a minimal $k$-separation of the matroid $M$. If either $\left(X-\mathrm{cl}_{M}(Y), \mathrm{cl}_{M}(Y)\right)$ or $\left(\mathrm{cl}_{M}(X), Y-\mathrm{cl}_{M}(X)\right)$ is a modular separation, then so too is $(X, Y)$.

Proof. Assume $\left(\mathrm{cl}_{M}(X), Y-\mathrm{cl}_{M}(X)\right)$ is a modular separation. First, by Lemma 7.3.3 $\lambda_{M}\left(\operatorname{cl}_{M}(X), Y-\mathrm{cl}_{M}(X)\right)=r_{M}(X)+r_{M}(Y-$ $\left.\mathrm{cl}_{M}(X)\right)-r(M)=r_{M}(X)+r_{M}(Y)-r(M)=\lambda_{M}(X, Y)$. Thus $r\left(\operatorname{cl}_{M}(X) \cap \operatorname{cl}_{M}\left(Y-\operatorname{cl}_{M}(X)\right)\right)=\lambda_{M}(X, Y) . \operatorname{But}_{M}\left(Y-\operatorname{cl}_{M}(X)\right) \subseteq \operatorname{cl}_{M}(Y)$ thus $r_{M}\left(\mathrm{cl}_{M}(X) \cap \mathrm{cl}_{M}(Y)\right) \geq \lambda_{M}(X, Y)$.

However, by submodularity we know that $r_{M}\left(\operatorname{cl}_{M}(X) \cap \mathrm{cl}_{M}(Y)\right) \leq r_{M}(X)+$ $r_{M}(Y)-r(M)=\lambda_{M}(X, Y)$. Thus $r_{M}\left(\operatorname{cl}_{M}(X) \cap \operatorname{cl}_{M}(Y)\right)=\lambda_{M}(X, Y)$.

Second, by hypothesis, $\operatorname{cl}_{M}(X) \cap \operatorname{cl}_{M}\left(Y-\operatorname{cl}_{M}(X)\right)$ is modular and round whose rank is $\lambda_{M}\left(X, Y-\mathrm{cl}_{M}(X)\right)$. By Lemma 7.3.3 $r_{M}(Y)=r_{M}\left(Y-\mathrm{cl}_{M}(X)\right.$. Therefore by Oxley [41] $\operatorname{cl}_{M}(Y)=\operatorname{cl}_{M}\left(Y-\mathrm{cl}_{M}(X)\right)$. Which in turn means that $\operatorname{cl}_{M}(X) \cap \operatorname{cl}_{M}\left(Y-\operatorname{cl}_{M}(X)\right)=\operatorname{cl}_{M}(X) \cap \operatorname{cl}_{M}(Y)$. Thus $\operatorname{cl}_{M}(X) \cap \operatorname{cl}_{M}(Y)$ is a round modular flat whose rank is $\lambda_{M}(X, Y)$. Thus $(X, Y)$ is a modular separation.

The next theorem is the main theorem of this section.

Theorem 7.4.4. If $(X, Y)$ is a minimal vertical $k$-separation of the supersolvably saturated matroid $M$, then $(X, Y)$ is a modular separation.

Proof. The proof is by induction on the rank of $M$. If $M$ has rank 1 , then the result holds vacuously. Say $M$ has rank 2 . Then a vertical $k$-separation $(X, Y)$ of $M$ must have $r_{M}(X)=r_{M}(Y)=1$, and $\lambda_{M}(X, Y)=0$. In this 
case $M$ is disconnected and $(X, Y)$ is clearly a modular separation.

Assume that $M$ has rank $t \geq 3$, and, for induction, that the theorem holds for all supersolvably saturated matroids whose rank is less than $t$. Since $M$ is supersolvable, $M$ has a maximal chain of modular flats. Hence $M$ has a modular hyperplane $H$. Consider the minimal vertical $k$-separation $(X, Y)$.

7.4.4.1. Either $X \subseteq H$ or $Y \subseteq H$.

Proof. Assume otherwise. Then $r_{M}(X \cup H)=r_{M}(Y \cup H)=r(M)$.

If the $k$-separation $(X \cap H, Y \cap H)$ of $M \mid H$ is not vertical, then we may assume that $\operatorname{cl}_{M \mid H}(X \cap H)=H$. Hence $\operatorname{cl}_{M}(X) \supseteq H$. Since $X \nsubseteq H$, it follows that $\mathrm{cl}_{M}(X)=E(M)$. But by Lemma 7.3.2, $r_{M}\left(Y-\mathrm{cl}_{M}(X)\right)=r_{M}(Y)$. Thus, $r_{M}(Y)=0$. But this is impossible which means we have a contradiction. Thus $X \subseteq H$ and the claim holds.

It follows that we may assume that the $k$-separation $(X \cap H, Y \cap H)$ of $M \mid H$ is vertical. Let $C^{*}=E(M)-H$. By Lemma 7.2.8 we may assume that $\operatorname{cl}_{M}\left(C^{*}\right) \cap H \subseteq \operatorname{cl}_{M}(X) \cap H$. By the same lemma $r_{M}\left(C^{*} \cup(X \cap H)\right)=$ $r_{M}(X \cap H)+1$. But $C^{*} \cap X \neq \emptyset$. Therefore $r_{M}\left(C^{*} \cup(X \cap H)\right)=r_{M}(X)$, so $C^{*} \subseteq \operatorname{cl}_{M}(X)$. Hence $Y-\operatorname{cl}_{M}(X) \subseteq H$. But, by Lemma 7.3.3, $r_{M}(Y-$ $\left.\operatorname{cl}_{M}(X)\right)=r_{M}(Y)$. Therefore $\operatorname{cl}_{M}\left(Y-\operatorname{cl}_{M}(X)\right) \supseteq Y . \operatorname{But}_{M}\left(Y-\operatorname{cl}_{M}(X)\right) \subseteq$ $H$. Therefore $Y \subseteq H$ and the claim follows.

By Claim 7.4.4.1, we may assume that $Y \subseteq H$. One possibility is that $\mathrm{cl}_{M}(Y)=H$ so that $\left(X-\mathrm{cl}_{M}(Y), \mathrm{cl}_{M}(Y)\right)=\left(C^{*}, H\right)$. By Lemma 7.3.4. $\left(C^{*}, H\right)$ is a minimal vertical $k$-separation of $M$, so, by Lemma 7.4 .2 , $\left(C^{*}, H\right)$ is a modular separation in $M$. By Lemma $7.4 .3(X, Y)$ is a modular separation of $M$.

The remaining case is that $\mathrm{cl}_{M}(Y)$ is a proper subset of $H$. 
7.4.4.2. If $\mathrm{cl}_{M}(Y)$ is a proper subset of $H$, then $(X \cap H, Y \cap H)$ is a vertical k-separation in $M \mid H$.

Proof. Assume otherwise. We know that $\mathrm{cl}_{M}(Y)$ is properly contained in $H$, so that $\mathrm{cl}_{M \mid H}(Y)$ is properly contained in $H$. Moreover, if $\operatorname{cl}_{M \mid H}(X) \supseteq H$, then $\operatorname{cl}_{M}(X) \supseteq Y$, contradicting the fact that $(X, Y)$ is a vertical $k$-separation of $M$. This proves the claim.

Now $(X, Y \cap H)$ is a vertical $k$-separation of $M \mid H$, and $(X, Y)=(X,(Y \cap H) \cup$ $\left.C^{*}\right)$ is a minimal vertical $k$-separation of $M$. It now follows from Lemma 7.3 .5 that $(X, Y \cap H)$ is a minimal vertical $k$-separation of $M \mid H$. By the induction assumption, $(X, Y \cap H)$ is a modular separation of $M \mid H$. It follows from this, and the fact that $\lambda_{M}(X, Y)=\lambda_{M \mid H}(X, Y \cap H)$, that $(X, Y)$ is a modular separation of $M$.

Consider the rank 6 matroid in Figure 7.3. This is the parallel connection of three copies of $F_{7}$ along different lines of a copy of $M\left(K_{4}\right)$. This matroid is supersolvably saturated. Observe the central flat $F$ which is isomorphic to $M\left(K_{4}\right)$. The separation of $F$ and any of the copies of $F_{7}$ is a minimal vertical separation and is also a modular separation. The guts of these separations is a 3-point line which is round and modular.

\subsubsection{Some Consequences of Modular Separations}

Now we show how a modular separation $(A, B)$ of a matroid $M$ and the flats $\operatorname{cl}_{M}(A)$ and $\operatorname{cl}_{M}(B)$ interrelate. We denote by $G(A, B)$ the intersection $\operatorname{cl}_{M}(A) \cap \operatorname{cl}_{M}(B)$.

Lemma 7.4.5. Let $M$ be a matroid with a separation $(A, B)$. If $(A, B)$ is a modular separation then $\mathrm{cl}_{M}(A)$ and $\mathrm{cl}_{M}(B)$ are modular flats.

Proof. We claim the following. 


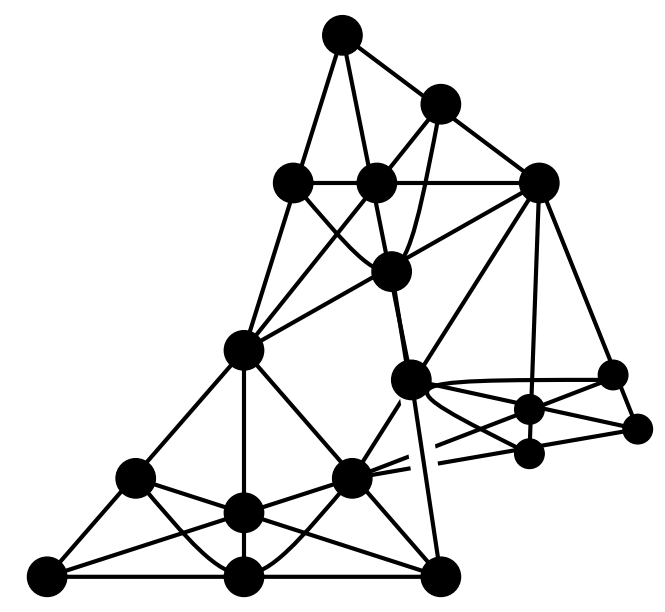

Figure 7.3: A rank 6, 18 element, supersolvably saturated matroid $M_{6,18}$

7.4.5.1. $r(M)-r_{M}\left(\mathrm{cl}_{M}(B)\right)=r_{M}\left(\mathrm{cl}_{M}(A)\right)-r_{M}(G(A, B))$.

Proof. We know because $(A, B)$ is a modular separation that $\lambda(A, B)=$ $r_{M}(G(A, B))$. Therefore we see that $r_{M}\left(\operatorname{cl}_{M}(A)\right)+r_{M}\left(\mathrm{cl}_{M}(B)\right)-r(M)=$ $r_{M}(G(A, B))$. If we rearrange this we obtain $r(M)-r_{M}\left(\operatorname{cl}_{M}(B)\right)=$ $r_{M}\left(\operatorname{cl}_{M}(A)\right)-r_{M}(G(A, B))$.

Now observe that $G(A, B)$ is modular in $M \mid \operatorname{cl}_{M}(B)$ since it is modular in $M$. Suppose $Z \subseteq B-\operatorname{cl}_{M}(A)$ is a flat of $M$. Then the following holds. We let $t=r(M)-r_{M}\left(\mathrm{cl}_{M}(B)\right)$ for $t \geq 0$.

7.4.5.2. $r_{M}\left(\operatorname{cl}_{M}(A) \cup Z\right)-r_{M}(G(A, B) \cup Z)=t$

Proof. Observe from 7.4.5.1 that $r_{M}\left(\mathrm{cl}_{M}(A)\right)-r_{M}(G(A, B))=r(M)-$ $r_{M}\left(\operatorname{cl}_{M}(B)\right)=t$. It follows that $r_{M}\left(\operatorname{cl}_{M}(A) \cup B\right)-r_{M}(G(A, B) \cup B)=$ $r(M)-r_{M}\left(\operatorname{cl}_{M}(B)\right)=t$. Now $\emptyset \subseteq Z \subseteq B-\operatorname{cl}_{M}(A)$ hence it must be that $t=r_{M}\left(\operatorname{cl}_{M}(A)\right)-r_{M}(G(A, B)) \leq r_{M}\left(\operatorname{cl}_{M}(A) \cup Z\right)-r_{M}(G(A, B) \cup Z) \leq$ $r_{M}\left(\mathrm{cl}_{M}(A) \cup B\right)-r_{M}(G(A, B) \cup B)=t$.

We want to show that $r_{M}\left(\operatorname{cl}_{M}(A)\right)+r_{M}(Z)=r_{M}\left(\operatorname{cl}_{M}(A) \cup Z\right)$. Clearly $\operatorname{cl}_{M}(A) \cap Z=\emptyset$. Furthermore we know that because $G(A, B)$ is modular 
then $r_{M}(G(A, B))+r_{M}(Z)=r_{M}(G(A, B) \cup Z)$.

By the previous claim $r_{M}\left(\operatorname{cl}_{M}(A) \cup Z\right)-r(G(A, B) \cup Z)=t$. So then $r_{M}\left(\operatorname{cl}_{M}(A) \cup Z\right)=t+r_{M}(G(A, B) \cup Z)=t+r_{M}(G(A, B))+r_{M}(Z)=$ $r_{M}(A)+r_{M}(Z)$. Hence $\operatorname{cl}_{M}(A)$ is modular in $M$. For the same reasons, as we have just seen that $\operatorname{cl}_{M}(A)$ is modular, it is obvious that $\operatorname{cl}_{M}(B)$ is also modular.

Lemma 7.4.6. Let $M$ be a matroid with a minimal vertical separation $(A, B)$. If $\operatorname{cl}_{M}(A)$ and $\operatorname{cl}_{M}(B)$ are modular flats then $\operatorname{cl}_{M}(A) \cap \mathrm{cl}_{M}(B)$ is a modular flat of $M$ and $\lambda_{M}(A, B)=r_{M}\left(\mathrm{cl}_{M}(A) \cap \mathrm{cl}_{M}(B)\right)$.

Proof. We know by Lemma 4.1.7 that if $\mathrm{cl}_{M}(A)$ and $\mathrm{cl}_{M}(B)$ are modular flats then so is their intersection. Thus we know that $\operatorname{cl}_{M}(A) \cap \mathrm{cl}_{M}(B)$ is modular.

Furthermore, $\lambda_{M}(A, B)=r_{M}\left(\operatorname{cl}_{M}(A)\right)+r_{M}\left(\operatorname{cl}_{M}(B)\right)-r(M)$ and $r_{M}\left(\mathrm{cl}_{M}(A) \cup\right.$ $\left.\mathrm{cl}_{M}(B)\right)=r_{M}(M)$. By the modularity of $\mathrm{cl}_{M}(A)$ and $\operatorname{cl}_{M}(B)$ it follows that $r_{M}\left(\operatorname{cl}_{M}(A)\right)+r_{M}\left(\operatorname{cl}_{M}(B)\right)=r_{M}\left(\operatorname{cl}_{M}(A) \cap \mathrm{cl}_{M}(B)\right)+r(M)$. Hence, $\lambda_{M}(A, B)=r_{M}\left(\operatorname{cl}_{M}(A) \cap \mathrm{cl}_{M}(B)\right)$.

Finally, we see that we can calculate rank across a modular separation as follows. First we give a technical result.

Lemma 7.4.7. Let $M$ be a matroid and let $(A, B)$ be a modular separation of $M$. Let $F_{1}, F_{2}$ be flats of $M$ such that $F_{1} \subset A$ and $F_{2} \subset B$. If $F_{1} \cap G(A, B)=$ $\emptyset=F_{2} \cap G(A, B)$ then $F_{1} \cup F_{2}$ is a flat.

Proof. Assume that $x \in c_{M}\left(F_{1} \cup F_{2}\right)$. Since $(A, B)$ partitions $E(M)$, we may assume that $x \in A$. Since $\operatorname{cl}_{M}(A)$ is modular, and we assumed $F_{2} \cap \operatorname{cl}_{M}(A)=\emptyset$, we have $r_{M}(A)+r\left(F_{2}\right)=r\left(A \cup F_{2}\right)$. Thus $M\left|\left(A \cup F_{2}\right)=M\right| A \oplus M \mid F_{2}$. This means that any circuit in $A \cup F_{2}$ that contains $x$ is contained in $A$, so from $x \in \operatorname{cl}_{M}\left(F_{1} \cup F_{2}\right)$ we get that 
$x \in \operatorname{cl}_{M}\left(F_{1}\right)$. That is, $x \in F_{1}$. Thus, $\operatorname{cl}_{M}\left(F_{1} \cup F_{2}\right)=F_{1} \cup F_{2}$, as needed.

Lemma 7.4.8. Let $M$ be a supersolvably saturated matroid. Let $(A, B)$ be a modular separation of $M$. Then $r_{M}(F)=r_{M}\left(F \cap \mathrm{cl}_{M}(A)\right)+r_{M}\left(F \cap \mathrm{l}_{M}(B)\right)-$ $r_{M}(F \cap G(A, B))$ for any flat $F$ of $M$.

Proof. Assume that $\left(F \cap \operatorname{cl}_{M}(A)\right) \cap\left(F \cap \operatorname{cl}_{M}(B)\right)$ is non-empty. Let $p$ be an element in $\left(F \cap \operatorname{cl}_{M}(A)\right) \cap\left(F \cap \mathrm{cl}_{M}(B)\right)$. We proceed by induction on the rank of $G(A, B)$. If $r_{M}(G(A, B))=0$ then $M$ is not connected. In this case, $r_{M}(F \cap G)=0$ so $r_{M}(F)=r_{M}\left(F \cap \operatorname{cl}_{M}(A)\right)+r_{M}\left(F \cap \mathrm{cl}_{M}(B)\right)$ which means that $M \mid F$ has a direct sum decomposition $M|F=M|\left(F \cap \mathrm{cl}_{M}(A)\right) \oplus M \mid\left(F \cap \mathrm{cl}_{M}(B)\right)$.

Next assume that $r_{M}(G(A, B))>0$. Let $G_{M / p}(A, B)=\mathrm{cl}_{M / p}(A) \cap \mathrm{cl}_{M / p}(B)$. By Lemma 4.1.10 if $\operatorname{cl}_{M}(A)$ is modular then $\mathrm{cl}_{M / p}(A)$ remains modular in $M / p$. This is also true for $\mathrm{cl}_{M / p}(B)$ and for $\mathrm{cl}_{M / p}(G(A, B))$. Now suppose that $r_{M / p}(F-p)=r_{M / p}\left((F-p) \cap \operatorname{cl}_{M / p}(A)\right)+r_{M / p}\left((F-p) \cap \mathrm{cl}_{M / p}(B)\right)-$ $r_{M / p}\left((F-p) \cap G_{M / p}(A, B)\right)$ holds.

Observe the following.

- $r_{M / p}(F-p)=r_{M}(F)-1$, and

- $r_{M / p}\left((F-p) \cap \mathrm{cl}_{M / p}(A)\right)=r_{M}\left(F \cap \mathrm{cl}_{M}(A)\right)-1$, and

- $r_{M / p}\left((F-p) \cap \mathrm{cl}_{M / p}(B)\right)=r_{M}\left(F \cap \mathrm{cl}_{M}(B)\right)-1$, and

- $r_{M / p}\left((F-p) \cap G_{M / p}(A, B)\right)=r_{M}(F \cap G(A, B))-1$.

This shows that $\left(r_{M}(F)-1\right)=\left(r_{M}\left(F \cap \mathrm{cl}_{M}(A)\right)-1\right)+\left(r_{M}(F \cap\right.$ $\left.\left.\operatorname{cl}_{M}(B)\right)-1\right)-\left(r_{M}(F \cap G(A, B))-1\right)$. But this implies that 
$r_{M}(F)=r_{M}\left(F \cap \operatorname{cl}_{M}(A)\right)+r_{M}\left(F \cap \operatorname{cl}_{M}(B)\right)-r_{M}(F \cap G(A, B))$.

Thus we conclude that for any flat of $M$ the equality must hold.

This means we can define the rank function for flats of supersolvably saturated matroids such as $M$ and therefore define rank across separations. Thus supersolvable saturation looks like a promising characterisation in our search for tree-like structure in matroids. Next we show that we can in fact obtain a canonical tree decomposition from a supersolvably saturated matroid. 


\section{Chapter 8}

\section{Tree Decompositions and Matroids}

In this chapter we establish some new results. Following a discussion of matroidal tree decompositions first introduced by Hliněný and Whittle [27], we show that for supersolvably saturated matroids we can establish a tree decomposition where the vertices are maximal round modular flats and the edges represent modular separations. Indeed, as we see, these tree decompositions can be treated as canonical.

\subsection{Matroidal Tree Decompositions}

Tree decompositions of graphs, being vertex-based, are not a natural fit with matroid theory but it is natural to ask if there is a type of treewidth that can be extended to matroids.

Hliněný and Whittle [27] have proposed such a type of treewidth. A VFtree-decomposition (where VF stands for Vertex Free) of a graph $G$ is a pair $(T, \tau)$ where $T$ is a tree and $\tau: E(G) \mapsto V(T)$ is an arbitrary mapping of edges to the tree nodes. For a node $x \in T$, denote the connected components 
of $T-x$ by $T_{1}, \ldots, T_{d}$ and the set $F_{i}=\tau^{-1}\left(V\left(T_{i}\right)\right)$. Then the node-width of $x$ is defined by

$$
|V(G)|+(d-1) \cdot k(G)-\sum_{i=1}^{d} k\left(G-F_{i}\right),
$$

where $k(H)$ denotes the number of components of a graph $H$. The width of the decomposition $(T, \tau)$ is the maximum width over all the nodes of $T$. The smallest width over all such tree decompositions of $G$ is the VF-tree-width of $G$. Observe that the width of an empty tree is 0 .

Now $\tau$ is not an analogue of the bag mapping $\beta: V(T) \mapsto 2^{V(G)}$ for standard tree decompositions of graphs. Instead $\tau$ replaces the condition of a tree decomposition that both ends of each edge are contained in some bag. The interpolation condition is embedded inside the node-width formula.

Theorem 8.1.1 (Hliněný and Whittle [27]). The tree-width of a graph $G$ equals the VF-tree-width of $G$.

We now show how to define vertex-free tree width for matroids [27]. Let $M$ be a matroid on the ground set $E=E(M)$. A pair $(T, \tau)$, where $T$ is a tree and $\tau: E \mapsto V(T)$ is an arbitrary mapping, is called a matroidal tree decomposition of $M$. For a node $x$ of $T$ we again denote the connected components of $T-x$ by $T_{1}, \ldots, T_{d}$ and the set $F_{i}=\tau^{-1}\left(V\left(T_{i}\right)\right) \subseteq E$. We then define the node-width of $x$ by

$$
\sum_{i=1}^{d} r_{M}\left(E-F_{i}\right)-(d-1) \cdot r(M) .
$$

The width of the decomposition $(T, \tau)$ is the maximal width over all the nodes of $T$. The smallest width over all the matroidal tree decompositions of $M$ is the matroidal treewidth of $M$. 
The following theorem relates treewidth with matroidal treewidth.

Theorem 8.1.2 (Hliněný and Whittle [27]). Let $G$ be a graph with at least one edge. Let $M=M(G)$ be the cycle matroid of $G$. Then the treewidth of $G$ equals the matroidal treewidth of $M$.

We now compare treewidth to branchwidth for matroids. We saw in the preliminaries that for a graph $G$ treewidth is bounded by the branchwidth of that graph. There is a similar result for matroidal treewidth.

Theorem 8.1.3 (Hliněný and Whittle [27]). Let $M$ be a matroid of matroidal treewidth $k$. Let $M$ have branchwidth $b$. Then

$$
b-1 \leq k \leq \max \{2 b-2,1\}
$$

\subsection{Tree Decompositions of Supersolvably Sat- urated Matroids}

Recall from earlier [23] that a clique tree of a chordal graph $G$ is a tree $T$ such that its vertices are the set of maximal cliques of $G$ and for each vertex $x \in V(G)$, the set of maximal cliques containing $x$ induces a subtree of $T$. Then the reduced clique graph of $G[23]$, denoted $C_{r}(G)$, has as its vertices the set of maximal cliques of $G$. An edge joins the cliques $U$ and $V$ if $U \cap V$ is a minimal separator of $G$ with the property that $U-V$ and $V-U$ are in different components of $G \backslash(U \cap V)$. Each edge of $C_{r}(G)$ is labeled with the order of the separation formed by its minimal separator.

Let $M$ be a supersolvably saturated matroid. We call a round modular flat of $M$ a rotunda. Then consider the analogue of the graphic structures in the previous paragraph for matroids. Let $T$ be a tree whose vertices are the set of maximal rotundas of $M$. Then $T$ is a rotunda tree for $M$ if for every element 
$x \in E(M)$ the set of maximal rotundas containing $x$ induces a subtree in $T$.

The rotunda graph of $M$ has as its vertices the set of maximal rotundas of $M$. An edge joins the maximal rotundas $U$ and $V$ if there is a modular separation $(X, Y)$ in $M$ with the properties that $U \subseteq \mathrm{cl}_{M}(X)$, and $V \subseteq \mathrm{cl}_{M}(Y)$, and $\operatorname{cl}_{M}(X) \cap \operatorname{cl}_{M}(Y)=U \cap V$. Moreover, each edge of this graph is labeled with the value $\lambda_{M}(X, Y)$. We denote the rotunda graph of $M$ as $R(M)$.

Let $F$ be a flat of $M$. Then the bridges of $F$ are the components of $M / F$.

Lemma 8.2.1. Let $(X, Y)$ be a modular separation in $M$. Then the bridges of $(X, Y)$ are the bridges of $\mathrm{cl}_{M}(X) \cap \mathrm{cl}_{M}(Y)$.

Proof. First, observe that the bridges of $(X, Y)$ are the components of $M / \mathrm{cl}_{M}(X)$ or of $M / \mathrm{cl}_{M}(Y)$ which are exactly the components of $M\left(Y-\mathrm{cl}_{M}(X)\right)$ or of $M \mid\left(X-\mathrm{cl}_{M}(Y)\right)$.

Let $G=\operatorname{cl}_{M}(X) \cap \mathrm{cl}_{M}(Y)$. The bridges of $G$ are the components of $M / G$.

8.2.1.1. In $M / G$ the partition $(X-G, Y-G)$ is a 1-separation.

Proof. Consider $\lambda_{M / G}(X-G)$. Then

$$
\begin{aligned}
& \lambda_{M / G}(X-G) \\
= & r_{M / G}(X-G)+r_{M / G}(Y-G)-r(M / G) \\
= & r_{M}(X \cup G)+r_{M}(Y \cup G)-\left(r(M)-r_{M}(G)\right)-2 r_{M}(G) \\
= & r_{M}(X \cup G)+r_{M}(Y \cup G)-r(M)-r_{M}(G)
\end{aligned}
$$

But we know because $(X, Y)$ is a modular separation that $r_{M}(G)=\lambda(X, Y)=$ 
$r_{M}(X)+r_{M}(Y)-r(M)$. Also remember that $G \subseteq \operatorname{cl}_{M}(X)$ and $G \subseteq \operatorname{cl}_{M}(Y)$. Given this we see that

$$
\begin{aligned}
& \lambda_{M / G}(X-G) \\
= & r_{M}(X \cup G)+r_{M}(Y \cup G)-r(M)-r_{M}(X)-r_{M}(Y)+r(M) \\
= & \left(r_{M}(X \cup G)-r_{M}(X)\right)+\left(r_{M}(Y \cup G)-r_{M}(Y)\right) \\
= & 0
\end{aligned}
$$

Given Claim 8.2.1.1, the components of $M / G$ are the components of $M \mid(X-$ $G)=M \mid\left(X-\operatorname{cl}_{M}(Y)\right)$ or $M|(Y-G)=M|\left(Y-\mathrm{cl}_{M}(X)\right)$. Observe that if we contract $X-\operatorname{cl}_{M}(Y)$ or $Y-\mathrm{cl}_{M}(X)$ the components of the other of side of $(X-G, Y-G)$ remain untouched. Thus the bridges of $(X, Y)$ are exactly the bridges of $G=\operatorname{cl}_{M}(X) \cap \operatorname{cl}_{M}(Y)$.

Lemma 8.2.2. Let $M$ be a supersolvably saturated matroid with rotunda graph $R(M)$. Let $(U, V)$ be an edge of $R(M)$. Let $(X, Y)$ be a modular separation such that $U \subseteq \operatorname{cl}_{M}(X)$ and $V \subseteq \operatorname{cl}_{M}(Y)$ and $\operatorname{cl}_{M}(X) \cap \operatorname{cl}_{M}(Y)=$ $U \cap V$. Then $U-V$ and $V-U$ are in different bridges of $M / \operatorname{cl}_{M}(X) \cap \mathrm{cl}_{M}(Y)$.

Proof. First observe that $U-V \subseteq X-\mathrm{cl}_{M}(Y)$. Thus $U-V$ is a component of $M / \mathrm{cl}_{M}(Y)$. Second, $V-U \subseteq Y-\mathrm{cl}_{M}(X)$. So $V-U$ is a component of $M / \mathrm{cl}_{M}(X)$. Therefore, $U-V$ and $V-U$ are in different bridges of $M / \mathrm{cl}_{M}(X) \cap \mathrm{cl}_{M}(Y)$.

Next we show that in supersolvably saturated matroids rotunda trees are equivalent to maximum weighted spanning trees of the rotunda graph. To achieve this we need a lemma that shows that if two maximal rotundas in the tree share a common element then every maximal rotunda along the unique 
path in between share this element.

Lemma 8.2.3. Let $M$ be a supersolvably saturated matroid. Let $T$ be a spanning tree of $R(M)$. Let $R_{i}$ and $R_{j}$ be maximal rotundas of $T$. Let $P_{i j}$ be the unique path of maximal rotundas between $R_{i}$ and $R_{j}$ in $T$. Let $x$ be an element of $E(M)$ that is in both $\mathrm{cl}_{M}\left(R_{i}\right)$ and $\mathrm{cl}_{M}\left(R_{j}\right)$. Then $x$ is in every maximal rotunda of $P_{i j}$.

Proof. Let $R_{k_{0}}$ denote $R_{i}$. Then $P_{i j}=R_{k_{0}}, \ldots, R_{k_{s}}, R_{j}$ is the sequence of maximal rotundas from $R_{i}$ to $R_{j}$. By induction on $s$ consider the following. As a base case we have that $x \in R_{k_{0}}=R_{i}$.

Now, for $0 \leq q \leq s$, since $R_{k_{q+1}}$ is next on the sequence $P_{i j}$ from $R_{k_{q}}$ to $R_{j}$, then there is an edge in $T$, and so in $R(M)$, between $R_{k_{q}}$ and $R_{k_{q+1}}$. By the definition of $R(M)$ this means there is a modular separation $(A, B)$ such that $\operatorname{cl}_{M}(A) \cap \operatorname{cl}_{M}(B)=R_{k_{q}} \cap R_{k_{q+1}}$. Observe that because $T$ is a tree then there can be no edge between $R_{k_{0}}$ and $R_{j}$. This means that $R_{k_{0}}, \ldots, R_{k_{q}} \subseteq \mathrm{cl}_{M}(A)$ and $R_{k_{q+1}}, \ldots, R_{j} \subseteq \mathrm{cl}_{M}(B)$.

Now $x \in R_{k_{q}}$ so $x \in \operatorname{cl}_{M}(A)$ and $x \in R_{j}$ so $x \in \operatorname{cl}_{M}(B)$. Thus $x \in \operatorname{cl}_{M}(A) \cap$ $\operatorname{cl}_{M}(B)=R_{k_{q}} \cap R_{k_{q+1}}$ And so if $x \in R_{k_{q}}$ then $x \in R_{k_{q+1}}$.

Theorem 8.2.4. Let $M$ be a supersolvably saturated matroid. Let $R(M)$ be its rotunda graph. Let $T$ be a spanning tree of $R(M)$. Then $T$ is a rotunda tree of $M$ if and only if $T$ is a maximum weighted spanning tree of $R(M)$.

Proof. First, we show that if $T$ is a rotunda tree then it is a maximum weighted spanning tree of $R(M)$. Suppose that $T$ is not a maximum weighted spanning tree. If $T$ is not a tree at all the result is trivial. If $T$ is not a spanning tree then it does not contain all the maximal rotundas in $M$ and so can not be a rotunda tree. So suppose that $T$ is a spanning tree of $R(M)$ but is not maximum weighted. 
Let $R_{i}$ and $R_{j}$ be two maximal rotundas that are adjacent in $R(M)$ but are not adjacent in $T$. Then there is a unique path $R_{i}, R_{k}, \ldots, R_{l}, R_{j}$ of maximal rotundas in $T$ from $R_{i}$ to $R_{j}$. Let $(X, Y)$ be a modular separation such that $R_{i} \subseteq \operatorname{cl}_{M}(X)$ and $R_{j} \subseteq \operatorname{cl}_{M}(Y)$ and $\operatorname{cl}_{M}(X) \cap \operatorname{cl}_{M}(Y)=R_{i} \cap R_{j}$. Let $(A, B)$ be a modular separation such that $R_{i} \subseteq \operatorname{cl}_{M}(A)$ and $R_{k} \subseteq \operatorname{cl}_{M}(B)$ and $\operatorname{cl}_{M}(A) \cap \operatorname{cl}_{M}(B)=R_{i} \cap R_{k}$.

Suppose the weight of the edge $\left(R_{i}, R_{j}\right)$ in $R(M)$ is higher than that of any of the edges $\left(R_{i}, R_{k}\right), \ldots,\left(R_{l}, R_{j}\right)$ in $R(M)$. This can happen because $T$ is not maximum weighted. Then we see that $\lambda_{M}(X, Y)>\lambda_{M}(A, B)$.

This means by the definition of modular separations that $r_{M}\left(\mathrm{cl}_{M}(X) \cap\right.$ $\left.\operatorname{cl}_{M}(Y)\right)>r_{M}\left(\operatorname{cl}_{M}(A) \cap \mathrm{cl}_{M}(B)\right)$. But we can see that this in turn means that $r_{M}\left(R_{i} \cap R_{j}\right)>r_{M}\left(R_{i} \cap R_{k}\right)$. As a result there must be an element $x \in E(M)$ such that $x \in\left(R_{i} \cap R_{j}\right)-\left(R_{i} \cap R_{k}\right)$. But then $x$ is in $R_{i}$ and $R_{j}$ but not in $R_{k}$. As a result $x$ does not induce the path $R_{i}, R_{k}, \ldots, R_{j}$ in $T$. Therefore $T$ is not a rotunda tree.

Second, we show that if $T$ is a maximum weighted spanning tree of $R(M)$ then $T$ is a rotunda tree. Assume that $T$ is a maximum weighted spanning tree of $R(M)$. Let $R_{i}$ and $R_{j}$ be two non-adjacent maximal rotundas of $T$ containing the element $x \in E(M)$. Consider the unique path $P_{i j}$ in $T$ between $R_{i}$ and $R_{j}$ including both $R_{i}$ and $R_{j}$. Then, by Lemma 8.2.3, $x$ must belong to every maximal rotunda in $P_{i j}$. Thus $x$ induces a subtree of $T$ and so $T$ is a rotunda tree.

This theorem has algorithmic implications for how we can efficiently find the treewidth of a supersolvably saturated matroid. However, for this to work we would need efficient ways to determine that a subset of the groundset is a rotunda and to construct the rotunda graph.

Next let us consider the tree decompositions of supersolvably saturated matroids. By the previous Theorem 8.2 .4 we know that if $M$ is a supersolvably 
saturated matroid then $M$ has a tree decomposition $T$ which is a maximum weighted spanning tree of $R(M)$. Observe that the width of $T$ will simply be the rank of the largest maximal rotunda in $M$.

We now show that a maximum weighted spanning tree of the rotunda graph of a supersolvably saturated matroid has the properties of a matroidal tree decomposition as per Hliněný and Whittle [27].

First we need further notation. Let $R_{x}$ be any maximal rotunda of a maximum weighted spanning tree of $R(M)$ for a matroid $M$. Let $T_{1}, \ldots, T_{d}$ be the subtrees formed by deleting $R_{x}$ from $T$. Let $F_{1}, \ldots, F_{d}$ be the bridges of $M / R_{x}$ and let $R_{y_{1}}, \ldots, R_{y_{d}}$ be rotundas in $T_{1}, \ldots, T_{d}$ that are adjacent to $R_{x}$.

Lemma 8.2.5. Let $M$ be a supersolvably saturated matroid. Let $T$ be a maximum weighted spanning tree of $R(M)$. Then for every maximal rotunda $R_{x} \in T$

$$
\left(\sum_{i=1}^{d} r_{M}\left(E-F_{i}\right)\right)-(d-1) r(M)=r_{M}\left(R_{x}\right)
$$

Proof. Let $F_{i}^{U}=\left(\bigcup_{k=1}^{d} F_{k}\right)-F_{i}$ and let $F_{i, \ldots, i+j}^{U}=\left(\bigcup_{k=1}^{d} F_{k}\right)-\left(\bigcup_{m=i}^{i+j} F_{m}\right)$.

Now observe that by definition $E-F_{i}=F_{i}^{U} \cup R_{x}$. By expansion of terms

$$
\begin{aligned}
& \left(\sum_{i=1}^{d} r_{M}\left(E-F_{i}\right)\right)-(d-1) r(M) \\
= & \left(r_{M}\left(F_{1}^{U} \cup R_{x}\right)+r_{M}\left(F_{2}^{U} \cup R_{x}\right)+\ldots+r_{M}\left(F_{d}^{U} \cup R_{x}\right)\right)-(d-1) r(M)
\end{aligned}
$$

8.2.5.1. $\mathrm{cl}_{M}\left(F_{i}^{U} \cup R_{x}\right)$ is a modular flat for all $i, 1 \leq i \leq d$. 
Proof. Because $M$ is supersolvably saturated and, by definition of $T$ as a spanning tree of $R(M)$, the edge $\left\{R_{x}, R_{y_{i}}\right\}$ displays the modular separation $\left(F_{i}, F_{1} \cup \ldots \cup F_{i-1} \cup F_{i+1} \cup \ldots \cup F_{d} \cup R_{x}\right)=\left(F_{i}, F_{i}^{U} \cup R_{x}\right)$. By Lemma 7.4.5 if $M$ is supersolvably saturated and $(A, B)$ is a modular separation of $M$ then $\operatorname{cl}_{M}(A)$ and $\mathrm{cl}_{M}(B)$ are both modular flats. Hence $\mathrm{cl}_{M}\left(F_{i}^{U} \cup R_{x}\right)$ is modular.

8.2.5.2. If for any $R_{x} \in T$ and for all $i, 1<i \leq d$,

$$
r_{M}\left(F_{1, \ldots, i-1}^{U} \cup R_{x}\right)+r_{M}\left(F_{i}^{U} \cup R_{x}\right)-r(M)=r_{M}\left(F_{1, \ldots, i}^{U} \cup R_{x}\right)
$$

Then

$r_{M}\left(F_{1}^{U} \cup R_{x}\right)+r_{M}\left(F_{2}^{U} \cup R_{x}\right)+\ldots+r_{M}\left(F_{d}^{U} \cup R_{x}\right)-(d-1) r(M)=r_{M}\left(R_{x}\right)$

Proof. Observe that rearranging the assumptive statement we obtain the following for $1<i \leq d$.

$$
r_{M}\left(F_{i}^{U} \cup R_{x}\right)=r_{M}\left(F_{1, \ldots, i}^{U} \cup R_{x}\right)-r_{M}\left(F_{1, \ldots, i-1}^{U} \cup R_{x}\right)+r(M)
$$

We see that most of these terms cancel out when we take the sum from 1 to $d$ of $i$ over $r_{M}\left(F_{i}^{U} \cup R_{x}\right)$ and we see that

$$
\begin{aligned}
& r_{M}\left(F_{1}^{U} \cup R_{x}\right)+r_{M}\left(F_{2}^{U} \cup R_{x}\right)+\ldots+r_{M}\left(F_{d-1}^{U} \cup R_{x}\right)+r_{M}\left(F_{d}^{U} \cup R_{x}\right) \\
= & r_{M}\left(F_{1, \ldots, d}^{U} \cup R_{x}\right)+(d-1) r(M)
\end{aligned}
$$

which means that 


$$
\begin{aligned}
& r_{M}\left(F_{1}^{U} \cup R_{x}\right)+r_{M}\left(F_{2}^{U} \cup R_{x}\right)+\ldots+r_{M}\left(F_{d}^{U} \cup R_{x}\right)-(d-1) r(M) \\
= & r_{M}\left(F_{1, \ldots, d}^{U} \cup R_{x}\right)+(d-1) r(M)-(d-1) r(M) \\
= & r_{M}\left(F_{1, \ldots, d}^{U} \cup R_{x}\right)
\end{aligned}
$$

But $F_{1, \ldots, d}^{U}=\emptyset$ so the result follows.

8.2.5.3. Given any node $x \in T$ then for all $i, 1<i \leq d$,

$$
r_{M}\left(F_{1, \ldots, i-1}^{U} \cup R_{x}\right)+r_{M}\left(F_{i}^{U} \cup R_{x}\right)-r(M)=r_{M}\left(F_{1, \ldots, i}^{U} \cup R_{x}\right) .
$$

Proof. For $1 \leq k \leq d$ then we know that $F_{k}^{U} \cup R_{x}$ is modular from step 8.2.5.1 so

$$
\begin{aligned}
& r_{M}\left(F_{1, \ldots, k-1}^{U} \cup R_{x}\right)+r_{M}\left(F_{k}^{U} \cup R_{x}\right) \\
= & r(M)+r_{M}\left(F_{1, \ldots, k}^{U} \cup R_{x}\right)
\end{aligned}
$$

Given these claims the result follows.

Now we present a central theorem for the thesis so far.

Theorem 8.2.6. Let $M$ be a supersolvably saturated matroid. Then $M$ has a minimum-width matroidal tree decomposition where each node is a maximal rotunda of $M$.

Proof. By Theorem 8.2.4 we know that $M$ has a tree decomposition $T_{R}$ where each node of $T_{R}$ is a maximal rotunda. By Lemma 8.2 .5 we know that for 
each node of $T_{R}$ the node-width is exactly equal to the rank of the maximal rotunda that is that node. Thus the matroidal treewidth for $T_{R}$ is exactly the width of $T_{R}$. Thus $T_{R}$ is a matroidal tree decomposition.

Moreover, the width of $T_{R}$ is the minimum width because the width of $T_{R}$ has to be greater or equal to the maximum node-width of any node of $T_{R}$ by definition. Here the maximum node-width is exactly equal to the largest rank of any maximal rotunda of $M$ so is exactly equal to the maximum node-width of any node of $T_{R}$.

Because this tree decomposition for a supersolvably saturated matroid $M$ is optimal we can treat it as a canonical tree decomposition of $M$. Consider the supersolvably saturated matroid given in Figure 8.1. This will have the rotunda tree given in Figure 8.2. Observe that the guts of the separations given for one separation by the dashed circle is a 3-point line. Each bag of the tree is a maximal round modular flat - either the Fano plane or $M\left(K_{4}\right)$.

Corollary 8.2.7. Let $M$ be a supersolvably saturated matroid. Let $t$ be the matroidal treewidth of $M$. Then for every maximal rotunda, $R$, of $M$ it follows that $r_{M}(R) \leq t$

Proof. We know from Theorem 8.2.6 that $M$ has a matroidal tree decomposition $T$ of minimal width $t$. From Lemma 8.2 .5 we can see that $r_{M}(R)$ is equal to the nodewidth at that point in $T$. Since the $r_{M}(R)$ must be at most the rank of the largest maximal rotunda of $M$ it follows that $r_{M}(R) \leq t$.

Further observe the following. Let $\omega(M)$ be the rank of the largest maximal rotunda of $M$ and let $b w(M)$ denote the branchwidth of $M$. Then by Theorem 8.1.3 (Hliněný and Whittle [27]) we see that

$$
b w(M)-1 \leq \omega(M) \leq \max \{2 b w(M)-2,1\} .
$$




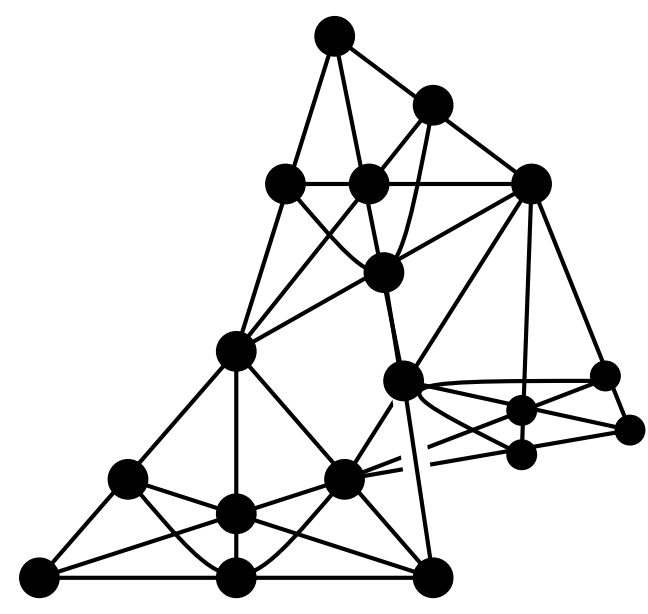

Figure 8.1: A rank 6, 18 element, supersolvably saturated matroid $M_{6,18}$

We see therefore that given a supersolvably saturated matroid $M$ of branchwidth $k$ there must be a rotunda tree of $M$ where the rank of the largest maximal rotunda is bounded by a function of $k$.

As a result, we can conclude that supersolvably saturated matroids have canonical tree decompositions of optimal treewidth in much the same way as chordal graphs have canonical tree decompositions where each bag is a clique of the graph. 


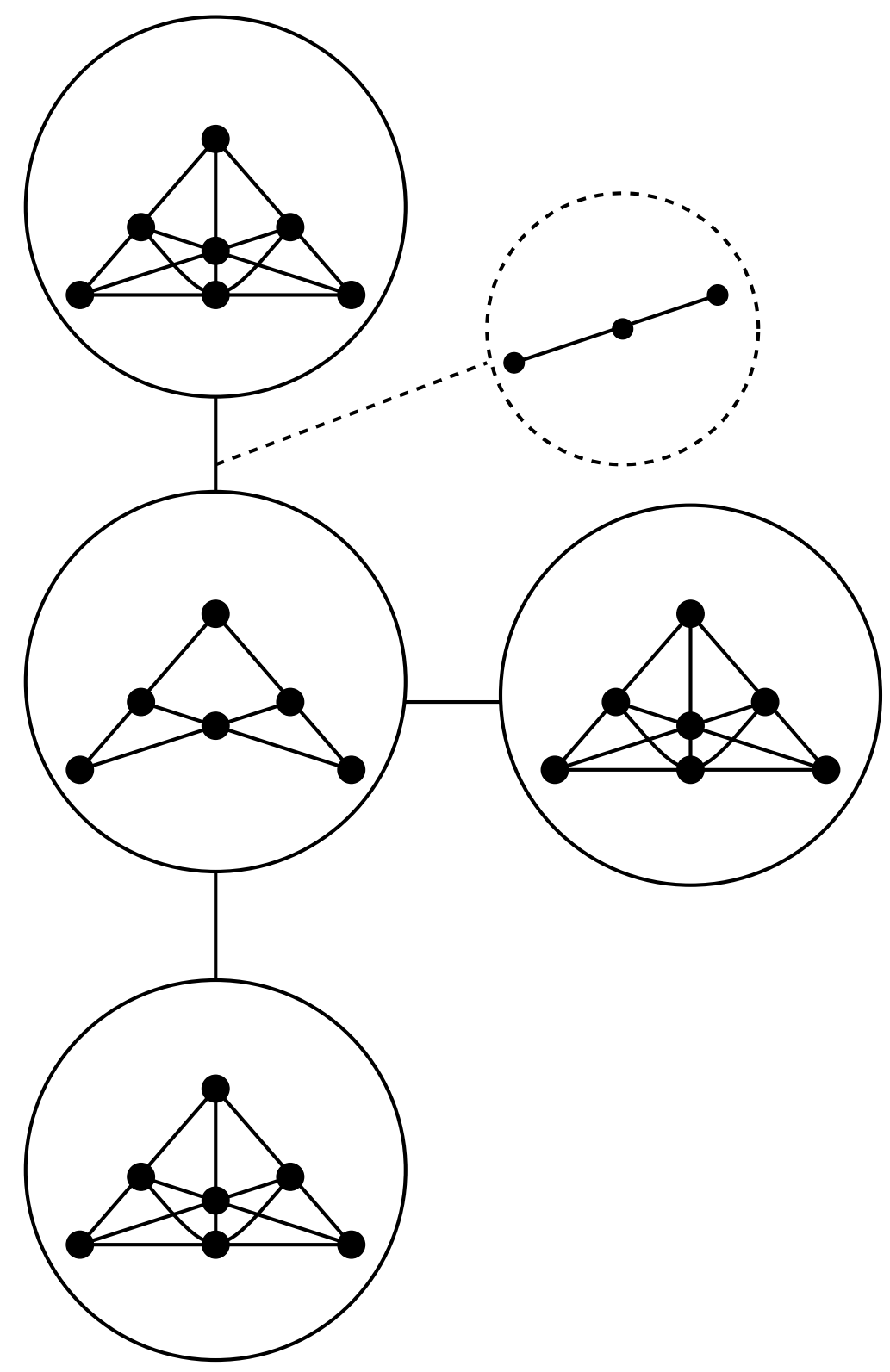

Figure 8.2: A rotunda tree for $M_{6,18}$ 


\section{Chapter 9}

\section{A Partial Converse to Hliněný}

In this final chapter, we tie together the results and discoveries of the previous chapters and show that we can establish a partial converse to Hliněný's Theorem [25].

\subsection{Establishing a Theorem}

We have been motivated by the problem described in Chapter 3, and in the course of this thesis, to prove the following conjecture.

Conjecture 9.1.1. Let $k$ be a positive integer and let $q$ be a prime power. Let $\mathcal{M}$ be a family of $G F(q)$-represented, supersolvably saturated, matroids all of whose members have branchwidth at most $k$. If membership of $\mathcal{M}$ can be decided by a finite state automaton then membership of $\mathcal{M}$ can be described in the logic $C M S_{q}$ over $G F(q)$-represented matroids.

This is a direct analogue of Theorem 1.1 of Bodlaender et al. [7] and is a partial converse to Hliněný's Theorem (Theorem 3.4.4) [25]. 
In the language of Hliněný [25], let $\mathcal{B}_{k}$ be the set of all $G F(q)$-represented matroids (for a given prime power $q$ ) that have branchwidth at most $k$ and so matroidal treewidth at most $k$. We can say that $\mathcal{M}$ is $k$-width finite state for $k \geq 1$ if $\mathcal{M} \cap \mathcal{B}_{k}$ is finite state. $\mathcal{M} \cap \mathcal{B}_{k}$ is finite state if the collection of all tree decompositions for the members of $\mathcal{M}$, bounded by branchwidth $k$, is accepted by some tree automaton.

We have seen that by Theorems 8.2.6 and 8.1.3 if a supersolvably saturated matroid $M$ has branchwidth at most $k$ then there is a rotunda tree whose largest maximal rotunda has rank bounded from above and below by $k$ and is thus optimal. Thus we can apply Lemma 3.4 .2 to supersolvably saturated matroids which means that we can apply the definition of $k$-width finite state. That is, $\mathcal{M} \cap \mathcal{B}_{k}$ is finite state if the collection of all optimal rotunda trees of width at most $k$ for the members of $\mathcal{M}$ is accepted by some tree automaton.

Next, we need to describe the counting monadic second-order logic for $G F(q)$ represented matroids that we call $C M S_{s}$ for some prime power $s$. This directly derives from Hliněný [25] and is as follows.

$C M S_{s}$ is defined over the class of $G F(s)$-represented matroids and includes the following symbols. We include variables $x_{1}, \ldots, x_{n}$ for individual points in the represented space that is vectors in the vector space. We further include $X_{1}, \ldots, X_{m}$ for point sets. We also include the constants $\emptyset, 0,1,2, \ldots$ as well as $E$.

$C M S_{s}$ is defined over a vector space and includes the usual vector space operators such as the span operator, $\langle$.$\rangle , and the dimension operator, \operatorname{dim}($.$) .$ We further include the function symbols $|\cdot|,\{\cdot\}, \cdot^{-}, \cup$ and $\cap$. In addition we have the relation symbols $=, \in, \subseteq$ as well as the logic symbols $\neg, \vee, \wedge, \exists, \forall$. The quantifiers $\forall, \exists$ are applicable to both point and set variables and the relation $=$ represents equality for both points and their sets. In addition we include the following predicates (or formulas): 
1. $\operatorname{indep}(F)$, where $F$ is a set variable, and the predicate is true iff $F$ is an independent set in the matroid.

2. $\operatorname{card}_{p, q}(X)$ means $|X| \bmod q=p$.

3. we write $\phi \rightarrow \psi$ to stand for $\neg \phi \vee \psi$.

4. in addition we add the following specific predicates for matroids derived from those above
i. $\quad X \supseteq Y \equiv \forall x(x \in X \vee x \notin Y)$
ii. $X \subset Y \equiv X \subseteq Y \wedge X \neq Y$
iii. $\neg X \equiv \forall x(x \notin X)$
iv. $\operatorname{basis}(B) \equiv \operatorname{indep}(B) \wedge \forall D(\neg(B \subseteq D) \vee(B=D) \vee \neg \operatorname{indep}(D))$
v. $\operatorname{basis}(B, F) \equiv(B \subseteq F) \wedge \operatorname{indep}(B) \wedge \forall D \wedge(D \subseteq F) \wedge(\neg(B \subseteq$ D) $\vee(B=D) \vee \neg \operatorname{indep}(D))$

Observe that this includes counting predicates as well as predicates for testing whether a set is a basis of the matroid or of a flat $F$ which we will need later.

Now we can begin establishing the following. Let $q$ be a prime power and let $k$ be a positive integer. Let $\mathcal{M}$ be a family of simple supersolvably saturated matroids represented over the finite field $G F(q)$ and of bounded matroidal treewidth $k$. We will show that for each $M \in \mathcal{M}$ we can obtain a tree decomposition that can be processed by a tree automaton. 
However, we have a problem. While the rank of every maximal rotunda is bounded by $k$ the cardinality of $M$ is not. This is a problem because any finite state automaton can only transition between a bounded number of states. To deal with this problem we need to colour the elements in each maximal rotunda with a bounded number of colours. This bound needs to be a function of $k$.

Consider $M \in \mathcal{M}$. The maximum number of elements in a maximal rotunda $F$ occurs when $M \mid F$ is isomorphic to a projective geometry. Given that $k$ is the rank of the largest maximal rotunda this number is bounded by $\frac{q^{k}-1}{q-1}<q^{k}$. We will now show that we can colour the rotundas of $M$ in at most $q^{k}$ colours.

\subsection{Colouring Supersolvable Matroids}

A colouring of a matroid is a function $f: E(M) \mapsto C$ where $C$ is a set of "colours". We say that $f$ is a proper l-colouring if $|C|=l$ and, for each rotunda $R$ of $M$, the function $\left.f\right|_{R}$ is injective. In other words, the colours are distinct for each rotunda.

Lemma 9.2.1. Let $q$ be a prime power and $k$ be a positive integer. Let $M$ be a supersolvably saturated $G F(q)$-represented matroid of matroidal treewidth at most $k$. Then $M$ has a proper $q^{k}$-colouring.

Proof. We proceed by induction on $r=r(M)$. As a base case, if $r(M) \leq k$ then the result is trivial. Suppose that $r(M)>k$. Then because $M$ has treewidth at most $k$ we know that $M$ has a vertical $k$-separation which, in turn, means, by Lemma 7.3.6, that there is a minimal vertical $k^{\prime}$-separation $(X, Y)$ of $M$ such that $k^{\prime} \leq k$.

Now by Lemma 7.4 .4 we know that $(X, Y)$ is a modular separation. This means that, by Lemma 7.4.5, $\mathrm{cl}_{M}(X), \mathrm{cl}_{M}(Y)$ are modular flats and $G=$ $\operatorname{cl}_{M}(X) \cap \operatorname{cl}_{M}(Y)$ is a rotunda. By Corollary 7.2 .5 we know that $M \mid \operatorname{cl}_{M}(X)$ 
and $M \mid \mathrm{cl}_{M}(Y)$ are supersolvably saturated matroids. We also know that $M \mid \mathrm{cl}_{M}(X)$ and $M \mid \mathrm{cl}_{M}(Y)$ are represented over $G F(q)$ and of bounded matroidal treewidth $k$ because so is $M$.

Since $(X, Y)$ is vertical, we see that $r_{M}(X)<r(M)$, and $r_{M}(Y)<r(M)$, so we can apply induction. Now hypothesize that $M \mid \mathrm{cl}_{M}(X)$ and $M \mid \mathrm{cl}_{M}(Y)$ both have proper $q^{k}$-colourings. In particular, observe that $G$ is a rotunda of both $M \mid \mathrm{cl}_{M}(X)$ and of $M \mid \mathrm{cl}_{M}(Y)$ and so has an injective $q^{k}$-colouring in each. The problem is that the two $q^{k}$-colourings may not be the same. So we permute the colours of $M \mid \mathrm{cl}_{M}(X)$ so that the $q^{k}$-colouring of $G$ in $M \mid \mathrm{cl}_{M}(X)$ matches the $q^{k}$-colouring of $G$ in $M \mid \mathrm{cl}_{M}(Y)$. Thus the $q^{k}$-colourings of $M \mid \mathrm{cl}_{M}(X)$ and $M \mid \mathrm{cl}_{M}(Y)$ induce a $q^{k}$-colouring of $M$. Now we look at $M$ as a whole.

9.2.1.1. If $F$ is a flat of $M$ such that $\left(X-\operatorname{cl}_{M}(Y)\right) \cap F \neq \emptyset \neq\left(Y-\operatorname{cl}_{M}(X)\right) \cap F$ then $F$ can not be round.

Proof. Let $(X \cap F, Y \cap F)$ be a vertical $k^{\prime}$-separation of $M \mid F$. Assume that $X \cap F$ spans $F$. Then, as $X \cap F \subseteq X \subseteq \operatorname{cl}_{M}(X)$, we see that $X$ spans $Y \cap F$, which means that $\left(Y-\operatorname{cl}_{M}(X)\right) \cap F=\emptyset$ which is contrary to the hypothesis. Thus $(X \cap F, Y \cap F)$ must be vertical which means that $M \mid F$ can not be round.

By Claim 9.2.1.1 we see that there can be no round flats of $M$ that cross $(X, Y)$. That is all rotundas of $M$ are either in $\mathrm{cl}_{M}(X)$ or in $\operatorname{cl}_{M}(Y)$. However, by the inductive hypothesis all the rotundas of $M \mid \mathrm{cl}_{M}(X)$ and of $M \mid \mathrm{cl}_{M}(Y)$ have injective $q^{k}$-colourings. As a result, we see that $M$ has a proper $q^{k}$-colouring.

Next we show that we can determine the rank function of $M$ given the rank function of each rotunda of $M$. 
Lemma 9.2.2. Let $q$ be a prime power and $k$ be a positive integer. Let $M$ be a supersolvably saturated $G F(q)$-represented matroid of branchwidth at most $k$. Then to determine the rank function of $M$ it suffices to know the rank function of each maximal rotunda of $M$.

Proof. We prove this lemma by induction on the $\operatorname{rank} r$ of $M$. As a base case consider a matroid $N$ consisting of a single element $x$ which will have rank 1 . Then $\emptyset$ is a hyperplane of this matroid with the modular separation $(\{x\}, \emptyset)$. Then the guts of this separation $D=\operatorname{cl}_{N}(\{x\}) \cap \emptyset=\emptyset$. Thus for every $F \subseteq\{x\}$ we see that $r_{N}(F)=r_{N}(F \cap \emptyset)+r_{N}\left(F \cap \mathrm{cl}_{N}(\{x\})\right)-r_{N}(F \cap \emptyset)=$ $r\left(F \cap \mathrm{cl}_{N}(\{x\})\right)$.

Let $H$ be a modular hyperplane of $M$ (which we know exists because $M$ is supersolvably saturated ) and $C^{*}$ be the complement of $H$ in $M$. Then $M \mid H$ is represented over $G F(q)$ and is supersolvably saturated by Corollary 7.2.5. Furthermore, by Lemma 7.4.2 $\left(C^{*}, H\right)$ is a modular separation of $M$. Then because $\left(C^{*}, H\right)$ is a modular separation we know that by definition $D=\mathrm{cl}_{M}\left(C^{*}\right) \cap H$ is a rotunda.

By induction we may assume we know the rank function of $M \mid H$. Let $r_{H}$ be the rank function of $M \mid H$. Because $\left(C^{*}, H\right)$ is a modular separation we know from Lemma 7.4 .5 that $\mathrm{cl}_{M}\left(C^{*}\right)$ is a modular flat. Now either $\operatorname{cl}_{M}\left(C^{*}\right)$ is a maximal rotunda or is contained in one. Let its rank function be $r_{C^{*}}$. Then we know from Lemma 7.4 .8 that $r_{M}(F)=r_{M}(F \cap H)+r_{M}\left(F \cap \operatorname{cl}\left(C^{*}\right)\right)-$ $r_{M}(F \cap D)$ for every $F \subseteq E$.

But we can see that $r_{M}\left(F \cap \mathrm{cl}_{M}\left(C^{*}\right)\right)=r_{C^{*}}\left(F \cap \mathrm{cl}_{M}\left(C^{*}\right)\right)$ and $r_{M}(F \cap D)=$ $r_{H}(F \cap D)$ (since also $D \subseteq H$ ). We can calculate these hence we can calculate the rank of $M$ for all subsets of $E(M)$.

Finally, a matroid $M$ together with a proper $q^{k}$-colouring is a $q^{k}$-coloured 
matroid. We can $q^{k}$-colour $M$ if $M$ has a proper $q^{k}$-colouring.

Theorem 8.2.6 and Lemma 9.2.1 show us that we have a tree decomposition for $M$ where the bags are coloured rotundas and the leaves are coloured maximal rotundas. Observe that by Lemma 9.2 .2 we can calculate the rank of any flat of $M$ given the ranks of the rotundas that constitute our tree decomposition. Thus the tree decomposition completely describes $M$.

Observe that this "coloured" tree decomposition is in fact a tree where the nodes are labeled from an alphabet of coloured matroids. This tree will act as our parse tree in the language of Hliněný [25] and of Mayhew [38]. We will run a leaf to root tree automaton (see Chapter 3 ) over this tree. We will talk more about this later. First, we need to show we can define this tree decomposition, and so our parse tree, in our logic $C M S_{q}$.

\subsection{Definability of Tree Decompositions}

We first show that the different components of our rotunda based tree decomposition are definable in this logic $C M S_{q}$.

Lemma 9.3.1. Let $q$ be a prime power and let $k$ be a positive integer. Let $M$ be a $G F(q)$-represented, supersolvably saturated, matroid with bounded branchwidth $k$. Then we can express in $C M S_{q}$ that a flat $F$ is modular.

Proof. By Lemma 4.1.12 a flat $F$ is modular if for every flat $Z$ and for every basis, $B u$, of $F \cup Z$ and for every basis, $B n$, of $F \cap Z, B n \subseteq B u$, then $B u \cap F$ is a basis of $F$ and $B u \cap Z$ is a basis of $Z$.

This can be expressed in the $C M S_{q}$ predicate "modular $(F) \equiv \forall Z[\forall B u \forall B n$ basis $(B u, F \cup$ $Z) \wedge \operatorname{basis}(B n, F \cap Z) \wedge B n \subseteq B u \wedge \operatorname{basis}(B u \cap F, F) \wedge \operatorname{basis}(B u \cap Z, Z)] "$.

Lemma 9.3.2. Let $q$ be a prime power and let $k$ be a positive integer. Let $M$ be a $G F(q)$-represented, supersolvably saturated, matroid with bounded 
branchwidth $k$. Then we can express in $C M S_{q}$ that a flat $F$ is a maximal rotunda.

Proof. A flat $F$ is a maximal rotunda if $F$ is round and modular and every set $Z$ that properly contains $F$ is not round. $F$ is round if, for every set $A$, if $A$ is a subset of $F$, then the separation $(A, F-A)$ is not vertical. This can be expressed in the $C M S_{q}$ predicate " $\operatorname{round}(F) \equiv \forall A(A \subseteq F \rightarrow$ $\left(\left(\exists A^{C} \wedge\langle A\rangle=A^{C} \wedge F-A \subseteq A^{C}\right) \vee\left(\exists F_{A}^{C} \wedge\langle F-A\rangle=F_{A}^{C} \wedge A \subseteq F_{A}^{C}\right)\right)$ ".

We can then apply Lemma 9.3.1 and define with a predicate maximal_rotunda that $F$ is both round and modular in the above sense and $Z$ is not round for every $Z$ containing $F$. That is "maximal_rotunda $(F) \equiv \operatorname{round}(F) \wedge$ $\operatorname{modular}(F) \wedge \forall Z(Z \supset F \rightarrow \neg \operatorname{round}(Z))$ ".

Lemma 9.3.3. Let $q$ be a prime power and let $k$ be a positive integer. Let $M$ be a $G F(q)$-represented, supersolvably saturated, matroid with bounded branchwidth $k$. Let $X$ be a flat of $M$. Then we can express in $C M S_{q}$ that $X$ is the guts of a modular separation.

Proof. $X$ is the guts of a modular separation if there exist $Y, Z$ such that $(Y, Z)$ is a partition and $Y, Z$ contain cocircuits and $e \in \operatorname{cl}(Y) \cap \operatorname{cl}(Z)$ for every $e \in E$ iff $e \in X$ and finally if $X$ is modular. We can define this in $C M S_{q}$ using the predicate "modular_guts $(X) \equiv \exists Y, Z(Y \subseteq X \wedge Z=$ $X-Y \wedge \exists C^{Y} \wedge \operatorname{cocircuit}\left(C^{Y}\right) \wedge C^{Y} \subseteq Y \wedge \exists C^{Z} \wedge \operatorname{cocircuit}\left(C^{Z}\right) \wedge C^{Z} \subseteq$ $Z \wedge \forall e\left(\exists Y^{C} \wedge\langle Y\rangle=Y^{C} \wedge \exists Z^{C} \wedge\langle Z\rangle=Z^{C} \wedge e \in Y^{C} \cap Z^{C} \leftrightarrow e \in\right.$ $X)) \wedge$ modular $(X) "$.

A cocircuit is the complement of a hyperplane and a hyperplane $H$ exists if there is no basis contained by $H$ and for every $x$ in $E-H$ there is a basis contained by $H \cup x$. This gives us the predicates "hyperplane $(H) \equiv$ $\left.\nexists B(\operatorname{basis}(B) \wedge B \nsubseteq H) \wedge \forall x \notin H\left(\exists B^{\prime} \wedge \operatorname{basis}\left(B^{\prime}\right) \wedge B^{\prime} \subseteq H \cup x\right)\right) "$ and "cocircuit $\left(C^{*}\right) \equiv$ hyperplane $\left(E-C^{*}\right)$ ". 
Lemma 9.3.4. Let $q$ be a prime power and let $k$ be a positive integer. Let $M$ be a $G F(q)$-represented, supersolvably saturated, matroid with bounded branchwidth $k$. Let $X$ and $Y$ be maximal rotundas of $M$. Then we can express in $C M S_{q}$ that $X$ is adjacent to $Y$.

Proof. $X$ and $Y$ are adjacent if $X$ and $Y$ are maximal rotundas, $G$ is the guts of a modular separation and $G$ is contained by both $X$ and $Y$.

Consider the following $C M S_{q}$ predicate. " $a d j(X, Y) \equiv$ maximal_rotunda $(X) \wedge$ maximal_rotunda $(Y) \wedge$ modular_guts $(G) \wedge G \subseteq X \wedge G \subseteq Y$ ". This is equivalent to showing that $X$ and $Y$ are adjacent.

Next we would want to prove the following.

Conjecture 9.3.5. Let $q$ be a prime power and let $k$ be a positive integer. Let $M$ be a supersolvably saturated matroid represented over the finite field $G F(q)$ and of bounded branchwidth $k$. Let $M$ have a proper $q^{k}$-colouring. Let $T$ be a minimal width tree decomposition of $M$. Then $T$ can be described by a sentence in the logic $C M S_{q}$ for matroids represented over $G F(q)$.

From the previous section we might think that we can construct a sentence in the logic $C M S_{q}$ describing a rotunda tree. We want to say that for all maximal round flats $X$ and $Y$ then $X$ and $Y$ are adjacent if there is a modular separation such that $\operatorname{cl}_{M}(X) \cap \mathrm{cl}_{M}(Y)$ is the guts of this separation.

We could try to do this by first guessing a set $Z$. We would then test that every element of $Z$ is a maximal rotunda. Finally, we test that all pairs of maximal rotundas as to whether they satisfy adjacency. They do not all have to be adjacent of course. 
We might do this with the following sentence using the above defined predicates "maximal_rotunda" and "adj".

Consider the sentence " $\forall X \in Z$ maximal_rotunda $(X) \wedge \forall X, Y \in Z[\operatorname{adj}(X, Y) \rightarrow$ maximal_rotunda $(X) \wedge$ maximal_rotunda $(Y)]$ ".

However, this approach fails for the following reason. Rotundas are sets of points of $M$. With monadic second-order logic we can list a bounded number, but we are not allowed to quantify over a general set of sets. To describe the whole rotunda tree or tree decomposition as in this lemma we need to quantify over all the maximal rotundas of which there is an unbounded though finite number. Addressing this problem will occupy the remainder of this chapter.

\subsection{Using an Extension Field}

We now introduce an approach to solving the problem described in the previous section. Let $G F\left(q^{n}\right)$ be a finite field extension of $G F(q)$. Since as we have seen we can $q^{k}$-colour $M$ we can bound the degree of our extension field by $k$.

As we have seen in the preceding section, we can not prove our original Conjecture 9.1.1. Instead we need the following theorem.

Theorem 9.4.1. Let $k$ be a positive integer and let $q$ be a prime power. Let $\mathcal{M}$ be a family of $G F(q)$-represented, supersolvably saturated, matroids all of whose members have branchwidth at most $k$. If membership of $\mathcal{M}$ can be decided by a finite state automaton then membership of $\mathcal{M}$ can be described in the logic $C M S_{q^{k}}$.

In the rest of this section we prove this theorem. First we show the equivalency between rotundas of the matroid over the original field $G F(q)$ and 
vector points over the extension field $G F\left(q^{k}\right)$. We need to understand how an extension field of $G F(q)$ means that every rotunda of $M$ has a representative point in $G F\left(q^{k}\right)$. Observe the following two lemmas.

Lemma 9.4.2. Let $q$ be a prime power and let $k$ be a positive integer. Let $P G(r-1, q) \subseteq P G\left(r-1, q^{k}\right)$ be projective geometries defined over finite fields $G F(q)$ and $G F\left(q^{k}\right)$. For every vector $v \in P G\left(r-1, q^{k}\right)$ there is a unique minimal subspace $Z \subseteq P G(r-1, q)$ such that $v \in\langle Z\rangle_{P G\left(r-1, q^{k}\right)}$.

Proof. First, $v$ must be in the span of some subspace of $P G(r-1, q)$ because it is defined over a vector space. Every subspace of $P G(r-1, q)$ is well-known to be modular. Next, consider any two such subspaces $A, B$. We show that if $v$ is in the span of both of these subspaces over the larger field then $v$ must be in the span of their intersection. By the rank of a subspace $Z$, that is $r(Z)$, in this context we mean the dimension of the span of that subspace, that is $\operatorname{dim}(\langle Z\rangle)$.

9.4.2.1. Let $A, B$ be subspaces of $P G(r-1, q)$. If $z \in\langle A\rangle_{P G\left(r-1, q^{k}\right)}$ and $z \in\langle B\rangle_{P G\left(r-1, q^{k}\right)}$ then $z \in\langle A \cap B\rangle_{P G\left(r-1, q^{k}\right)}$.

Proof. Because $A, B$ are modular we know that $r(A \cap B)=r(A)+r(B)-$ $r(A \cup B)$. In particular by submodularity we know that $r(A \cap B) \leq r(A)+$ $r(B)-r(A \cup B)$. If we add $z$ we obtain $r((A \cap B) \cup z) \leq r(A \cup z)+$ $r(B \cup z)-r((A \cup B) \cup z)$. But $r(A \cup z)=r(A), r(B \cup z)=r(B)$ and $r((A \cup B) \cup z)=r(A \cup B)$ because of our assumption that $z \in\langle A\rangle_{P G\left(r-1, q^{k}\right)}$ and $z \in\langle B\rangle_{P G\left(r-1, q^{k}\right)}$. Thus we see that $r((A \cap B) \cup z) \leq r(A \cap B)$. But clearly $r((A \cap B) \cup z) \geq r(A \cap B)$. Thus $z \in\langle A \cap B\rangle_{P G\left(r-1, q^{k}\right)}$.

Since the intersection $A \cap B$ spans $A$ and $B$ over the larger field we see that for each pair of subspaces $A$ and $B$ there is a single subspace which spans $v$ over $P G\left(r-1, q^{k}\right)$. Since this is true for any two such subspaces there must be a unique subspace $Z \subseteq P G(r-1, q)$ which spans $v$ over $P G\left(r-1, q^{k}\right)$ and $Z$ will be minimal by definition. 
Lemma 9.4.3. Let $q$ be a prime power and let $k$ be a positive integer. Let $P G(r-1, q) \subseteq P G\left(r-1, q^{k}\right)$ be projective geometries defined over finite fields $G F(q)$ and $G F\left(q^{k}\right)$. For every subspace $Z \subseteq P G(r-1, q)$ such that $r(Z) \leq k$ there is a vector $v \in P G\left(r-1, q^{k}\right)$ such that the unique minimal subspace of $P G(r-1, q)$ that spans $v$ is $Z$.

Proof. Let $b_{1}, \ldots, b_{k}$ be a basis of $Z$. Because of our choice of extension field $G F\left(q^{k}\right)$, that is if $k$ is large enough, there is an element $\alpha \in G F\left(q^{k}\right)-G F(q)$ which is the root of a degree $k$ polynomial with coefficients in $G F(q)$ and is not the root of any such lower degree polynomial. We can then construct a vector $v$ as follows. Let $v=b_{1}+\alpha b_{2}+\alpha^{2} b_{3}+\ldots+\alpha^{k-1} b_{k}$. We see that $v$ is in $P G\left(r-1, q^{k}\right)$ and is spanned by $b_{1}, \ldots, b_{k}$. Moreover, $v$ is the minimal such vector since if we reduce the basis by any vector $b_{i}, 1 \leq i \leq k$, and so reduce the rank then the basis will not span this vector over the larger field.

Let $q$ be a prime power and let $k$ be a positive integer. Let $\mathcal{M}$ be a family of $G F(q)$-represented, supersolvably saturated, matroids with bounded branchwidth $k$. Consider $M \in \mathcal{M}$. We know that $M$ is represented over $G F(q)$ and there is an extension field $G F\left(q^{k}\right)$ that contains $G F(q)$. Certainly $M \subseteq P G(r-1, q)$. Thus every maximal rotunda $R$ of $M$ is a subspace of $P G(r-1, q)$. Moreover the rank of $R$ is at most $k$ as we have shown in Corollary 8.2.7. Thus we can apply Lemma 9.4 .3 to see that there must be a point $v$ over $G F\left(q^{k}\right)$, and so in $P G\left(r-1, q^{k}\right)$, for which $R$ is the unique and minimal subspace which spans $v$.

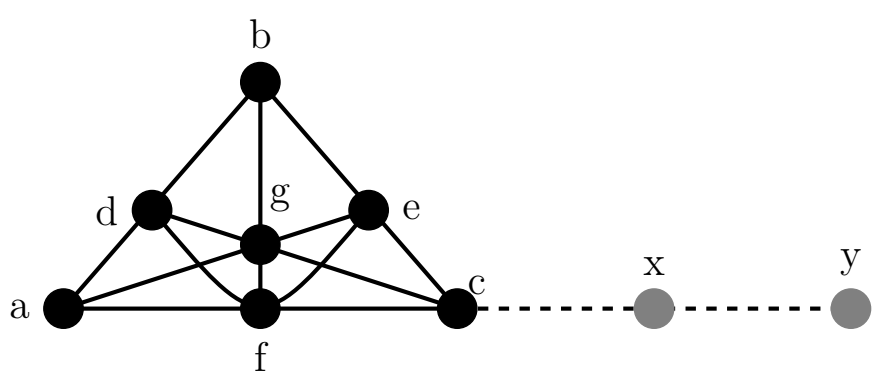

Figure 9.1: Fano plane $F_{7}$ or $P G(2,2)$ with extension line 
To illustrate the above consider Figure 9.1. Here we have the Fano plane or the matroid $P G(2,2)$ but this lives in an extension field where every line has 5 points. For example, we can see that the point $x$ is spanned by the unique round modular flat $\{a, c, f\}$. Similarly, $\{a, c, f\}$ spans both $x$ and $y$. From now on when we use the term "flat" this is intended to be synonymous with subspace.

We need a further lemma that shows we can describe in the logic $C M S_{q^{k}}$ that a point over $G F\left(q^{k}\right)$ is a representative of a flat of $M$.

Lemma 9.4.4. Let $q$ be a prime power and let $k$ be a positive integer. Let $M$ be a $G F(q)$-represented, supersolvably saturated, matroid with bounded branchwidth $k$. Let $p$ be a point over $G F\left(q^{k}\right)$ and let $X$ be a subset of $E(M)$. Then we can express in $C M S_{q^{k}}$ that $p$ is minimally in the span of $X$.

Proof. We express this as follows. For every $X \subseteq E$ there exists $p \in P G(r-$ $\left.1, q^{k}\right)$ such that $p \in\langle X\rangle_{P G\left(r-1, q^{k}\right)}$ and for every $Z$ such that $Z \subseteq P G(r-1, q)$ and $\langle Z\rangle_{P G\left(r-1, q^{k}\right)} \subsetneq\langle X\rangle_{P G\left(r-1, q^{k}\right)}$ we have that $p \notin\langle Z\rangle_{P G\left(r-1, q^{k}\right)}$.

We now begin the proof of Theorem 9.4.1.

Proof. First, we want to use Courcelle's Theorem for Trees [15] to show that if a labeled tree is recognisable by a standard tree automaton then it is describable by a sentence in the counting monadic second-order logic of such trees. Thus we need to construct a labeled tree given $M$.

By Theorem 8.2.6 $M$ has a minimum-width tree decomposition $T$ that is a rotunda tree. Moreover, by Lemma 9.2.1, $M$ has a proper $q^{k}$-colouring so every rotunda of $T$ can be coloured with at most $q^{k}$ colours. Let $T_{L}$ be a tree with a vertex for every maximal rotunda of $T$. Let $X, Y$ be maximal rotundas of $M$ and let $x, y$ be the corresponding vertices in $T_{L}$. Then 
$\{x, y\}$ is an edge if there is a modular separation $(A, B)$ of $M$ such that $G(A, B)=\operatorname{cl}_{M}(X) \cap \operatorname{cl}_{M}(Y)$. Finally, we designate one vertex, $r$, of $T_{L}$ as the root vertex.

We label $T_{L}$ as follows. Consider a ranked alphabet $\mathcal{A}$ consisting of all the possible $q^{k}$-coloured matroids we can construct from the rotundas of $M$ individually and by joining them together. Let $n$ be the upper bound on the number of such coloured matroids. $n \approx\left(2^{q^{k}}\right)\left(q^{k}\right)^{q^{k}}$ because for each rotunda there are $2^{q^{k}}$ possible subsets and $q^{k^{q^{k}}}$ possible combinations of rotundas. Then we can list the elements of $\mathcal{A}$ as $\left\{\alpha_{1}, \ldots, \alpha_{n}\right\}$. Each $\alpha_{i}, 1 \leq i \leq n$, corresponds to a $q^{k}$-coloured matroid. In particular, each $q^{k}$-coloured rotunda $R_{j}$ of $T$ corresponds to a letter $\alpha_{j}$ of $\mathcal{A}$.

Thus we label each vertex $v_{i}$ of $T_{L}$ with the label $\alpha_{i}$ that matches the $q^{k}$ coloured maximal rotunda $R_{i}$ that corresponds to $v_{i}$. We add to each label of $T_{L}$ for each vertex an indicator as to whether this vertex is the root vertex.

There will be an associated counting monadic second-order logic for labeled trees that has as its domain the labels of $\mathcal{A}$. This operates in exactly the same way as one would expect for the logic for trees and as used by Courcelle in his theorem for trees [15]. We will call this $C M S_{T}$.

Given this we can apply Courcelle's Theorem for Trees [15]. Suppose we have a labeled tree $T_{L}$ of the sort we just showed we can construct from $M$. By the theorem if a tree automaton can recognise this tree then it is describable by a sentence in $C M S_{T}$.

We follow Hliněný [25] closely here to see that such a tree should be recognisable. Essentially we have a $k$-boundaried parse tree in Hliněný's language [25]. The automaton starts at the leaves and traverses up the tree joining together the $q^{k}$-coloured matroids represented by each label using Hliněný's composition operators. When the tree automaton reaches the root vertex, if 
the resulting matroid is a member of $\mathcal{M}$, that is of supersolvably saturated matroids bounded by branchwidth $k$ and represented over $G F(q)$ with an extension field $G F\left(q^{k}\right)$, then the automaton accepts.

Now, we need to show that any sentence $\phi$ over a language of $q^{k}$-coloured matroids, $C M S_{T}$, has a corresponding sentence $\phi^{*}$ in the logic $C M S_{q^{k}}$ over $\mathrm{GF}(\mathrm{q})$-represented, supersolvably saturated, matroids of bounded branchwidth $k$ over $G F\left(q^{k}\right)$.

However, any statement about a matroid $M$ can be expressed in terms of its rank function [41]. By Lemma 9.2.2 we know that given the rank function of each $q^{k}$-coloured rotunda of $M$ we can determine the rank function of $M$. Thus we can express any sentence describing a relationship between the vertices and edges of $T_{L}$ as a sentence about $M$.

We then use the lemmas we introduced earlier in section 9.3. These still apply over $C M S_{q^{k}}$ since every point that belongs to a vector space over $G F(q)$ also belongs to a vector space over $G F\left(q^{k}\right)$ though in some cases we might need to restrict to the groundset $E$. In particular, we use Lemma 9.3 .2 to identify a flat as a maximal rotunda and Lemma 9.3.4 to identify two rotundas as adjacent in $T$ of $M$.

We discover, in doing this, that we seem to be allowing for sentences which quantify over sets of rotundas. Our logic appears too strong. This is in fact not the case when we take account of our use of an extension field. By Lemma 9.4.3 for every rotunda of $M$ there is a point $v$ over $G F\left(q^{k}\right)$ that is in the span of that rotunda. This means that any sentence in $C M S_{q^{k}}$ expressing something about a rotunda $R$ of $M$ can be rewritten as a sentence about a single point over $G F\left(q^{k}\right)$. We use Lemma 9.4.4 to express in $C M S_{q^{k}}$ that a rotunda of $M$ is represented by a single point over $G F\left(q^{k}\right)$.

With the above we see that given $M$ we can construct a labeled tree $T_{L}$ over 
the logic $C M S_{T}$. We can apply Courcelle's Theorem for Trees [15] to see that if any such tree $T_{L}$ is recognisable it can be described by a sentence in $C M S_{T}$. Then we see that any sentence in $C M S_{T}$ has a corresponding sentence in $C M S_{q^{k}}$ which quantifies over points over $G F\left(q^{k}\right)$ rather than over sets of rotundas.

To paraphrase Bodlaender et al. [7] quantification over elements of $T_{L}$ directly translates to quantification over points of $M$. That is we see that a sentence $\phi$ in $C M S_{T}$ that expresses whether an automaton accepts on $T_{L}$ can be translated to a sentence $\phi^{*}$ in $C M S_{q^{k}}$ such that $\phi$ holds for the labeled tree $T_{L}$ if and only if $\phi^{*}$ holds for $M$.

However, there is a weakness with this approach. We have narrowed the class of matroids we are proving our result for and have overly strengthened the logic we are using by broadening the domain from points over $G F(q)$ to points over an extension field of $G F(q)$. This means that our result is a much weaker partial converse to Hliněný's Theorem [25] than we would desire. However, the result is still an analogue to Theorem 1.1 of Bodlaender et al. [7]. 


\section{Chapter 10}

\section{Conclusions}

Our original aim was to prove a partial converse to Hliněný's Theorem [25] for matroids represented over a finite field. This theorem shows that for matroids of this class it is possible to recognise members of the class if it is possible to describe the class using a counting monadic second-order logic provided the matroids have bounded branchwidth.

However, most of this thesis has been devoted to determining the appropriate structure to require of our matroids. We investigated modularity and roundness. We observed that for graphs if they are chordal one can obtain a special highly structured tree of cliques. We realized that such a tree decomposition was exactly the sort of structure we needed.

Given this we decided to examine chordality for matroids. We carefully chose five definitions of chordality in matroids ranging from the very weak to the very strong. We observed that for binary and for graphic matroids these definitions are equivalent but for general matroids they differ - sometimes to a significant degree. While interesting we realized that even strong chordality for matroids does not give us the structure we need.

However, there is a result originally given by Dirac [18] and Stanley [47] 
that shows that supersolvability in the cycle matroids of graphs is equivalent to graph chordality. As a result we turned our attention to supersolvability. This looked more promising given that it involves a matroid having a chain of modular flats. Unfortunately, this is not enough. In any tree decomposition we construct we want the intersection of the bags to be round and modular. Supersolvability does not guarantee that every round flat is modular. As a result, we have to require that our matroids be supersolvably saturated.

We saw that supersolvable saturation is exactly the sort of structure we need. The only issues left included the important requirement that our separations be well behaved. However, as we have shown, in supersolvably saturated matroids, any minimal vertical $k$-separation is what we call a modular separation and is well-behaved. We can capture the concept of the guts as a round modular flat whose rank will be exactly the connectivity of the separation.

This then allows us to construct a well-behaved tree decomposition for any supersolvably saturated matroid. As we see every supersolvably saturated matroid has a graph of round modular flats which we call rotundas. We can apply an algorithm to find a maximum weighted spanning tree of the graph. Any such tree is an optimal tree decomposition for the matroid. It is a matroidal tree decomposition in the language of Hliněný and Whittle [27] such that its treewidth is exactly the rank of the largest rotunda in the tree. Furthermore, this maximum rank is bounded from above and below by the branchwidth of the matroid.

We were now able to turn our attention back to the original problem of this thesis. We start with a finite state matroid which is supersolvably saturated, represented over $G F(q)$ for some prime power $q$, and of bounded branchwidth $k$. This allows us to construct a canonical tree decomposition for the matroid. Then we need to show that we can run a tree automaton over our tree and describe it in a sentence of our particular counting monadic secondorder logic for matroids represented over $G F(q)$ which we call $C M S_{q}$. Here 
we encountered a first problem. While we are given a bounded rank for each rotunda the actual cardinality of the matroid is not bounded. We need this to be bounded so we can use a finite state tree automaton. We solve this problem by colouring the points in each rotunda so that each uses at most $q^{k}$ colours. We call this a proper $q^{k}$-colouring of $M$.

Next we proceeded to define the separate elements of the rotunda tree. We can define flats that are round, modular and maximal. We can define that a separation is modular. We can define that bags in the tree are adjacent to each other. However, we then encounter a major problem. To completely describe the rotunda tree in $C M S_{q}$ we need to be able to quantify over the rotundas. Because the number of rotundas is unbounded this means quantifying over a set of sets. This unfortunately is not allowed in monadic second-order logic.

We realized we could overcome this problem by using extension fields. For this we changed our matroid class requirements so that our matroids are defined over a finite field which is contained inside a larger extension field of the finite field. We could then take advantage of the fact that given a flat, $F$, of points for $M$ there will be a point, $z$, in the extension field in the span of $F$. That is $z$ is not in $F$ itself so can be considered free. We can use this structure to give us a representative for each maximal rotunda of our matroid $M$

We then construct a labeled tree where the labels correspond to $q^{k}$-coloured matroids derived from $M$. This tree operates over a counting monadic second-order logic for trees we call $C M S_{T}$. Essentially this tree is a $k$ boundaried parse tree in the language of Hliněný [25].

As a result, we are able to apply Courcelle's Theorem for Trees [15] over this tree showing that if the tree is recognisable it is definable in the logic $C M S_{T}$. We then show that given the rank function for the $q^{k}$-coloured rotundas of $M$ that correspond to the labels of our labeled tree we can calculate the rank 
function of $M$.

Thus any sentence in $C M S_{T}$ corresponds to a sentence over the $C M S_{q^{k}}$ of $G F(q)$-represented, supersolvably saturated, matroids of branchwidth at most $k$ with an extension field $G F\left(q^{k}\right)$. We avoid the problem of having to quantify over sets of rotundas by using the fact that each rotunda has a representative point over $G F\left(q^{k}\right)$.

There are some problems with this approach. First we have had to narrow our class of matroids further to require an extension field. Second, our logic is too strong. We have had to generalize the domain over which the logic is defined. Because of this our result is not as good a partial converse to Hliněný's Theorem as we could desire. It is however a direct analogue to a Theorem (Theorem 1.1) of Bodlaender et al. [7].

To conclude, we examined structure in matroids which is parallel to chordality in graphs. We saw that a number of related concepts of chordality are fragmented for general matroids while equivalent for binary and graphic matroids. We showed that given a supersolvably saturated matroid one can construct a very nicely behaved canonical tree of round modular flats. Given this we can show that for such matroids recognisability implies definability in monadic second-order logic.

But for this we require two things. First, we require that the class of supersolvably saturated matroids be represented over a finite field and that there be an extension field of this. Second, we must extend our counting monadic second-order logic to be defined over the extension field. 


\subsection{Open Questions}

This leaves some open questions. These are as follows.

1. We would like to know exactly how C-chordality, and indeed Bonin chordality, relate to strong chordality.

2. We would like to know if modular spanned flats of a matroid are always round flats.

3. We need to find a better strategy for assigning representatives to each maximal rotunda. We need this to not require use of any special structure such as extension fields.

We have investigated an alternate strategy using a concept called a guidance system borrowed from Bojanczyk and Pilipczuk [8]. Our idea was to assign to every 3-point line in a supersolvably saturated matroid $M$ a "root" element. We would then show that for every round modular flat $F$ of $M$ there is an element $u$ such that if we take the union of all the "root" elements for every line on which $u$ lies the closure of this will contain $F$. This would then give us a way to represent $F$ by a single element in $E(M)$.

Unfortunately, this strategy doesn't work if $u$ is in the intersection of maximal round modular flats. It is possible for any rotunda tree for $M$ to be such that some of its maximal rotundas are completely covered by intersections with other rotundas. One approach to address this problem would be to further require that our rotunda tree decompositions be what Bojanczyk and Pilipczuk [8] call "sane" tree decompositions. This would mean, among other things, that every maximal rotunda of a rotunda tree would be required to contain at least one element that is not in the intersection with other maximal rotundas. 
4. Given an answer to 3, we would like to know if we can generalise the result to more general classes of matroid. This would involve finding a way to construct nicely behaving tree decompositions for the matroids. That is prove the general converse to Hliněný's Theorem [25].

5. Finally, we can ask how similar the concept of the modular separation is to the Generalised Parallel Connection for matroids? 


\section{Index}

$\sqcap, 54$

$q^{k}$-colour, 139

$q^{k}$-coloured, 138

acyclic graph, 8

adjacent, 8

alphabet, 20

atomic, 21

bags, 12

bases, 9

basis, 10

binary, 34

Bonin chordal, 74

bound, 22

branch decomposition of a graph, 14 branch decomposition of a matroid,

15

branchwidth of a graph, 14

branchwidth of a matroid, 17

canonical right congruence, 27

chain, 93

child, 8

chord, 63

chordal, 64

circuits, 9

clique cut, 64

colouring, 136

complete graph, 8

connected graph, 8 connected matroid, 10

connectivity function, 10

contraction, 8

cut vertex, 63

cycle, 8

cycle matroid, 9

definable, 30

depth, 22

deterministic finite automaton, 25

domain, 20

edge width for a graph, 14

edge width for a matroid, 17

effectively, 32

finite index, 27

finite state, 27

free, 22

free tree, 8

graph, 7

guts, 111

height, 30

induced, 63

induces, 63

isomorphism, 8

leaf to root tree automaton, 38

letter, 20

line, 49 
loopless, 11

matroid, 9

matroidal tree decomposition, 120

matroidal treewidth, 120

maximal chain, 93

maximal clique, 64

minimal modular flat, 56

minimal vertex cut, 63

minor, 8

modular, 45

modular matroid, 49

modular pair, 45

modular separation, 111

modular set, 51

necessary, 56

neighbours, 8

optimal, 14

parent, 8

parse tree, 139

partial equipment signature, 40

partially equipped, 40

path, 7

perfect elimination ordering, 41

planar graph, 8

point configuration, 11

position, 20

projective transformation, 10

projectively equivalent, 11

rank, 9

ranked, 29

recognisable, 26

recursively supersolvable, 94

represent, 10 represented, 10

right congruence, 26

root, 8

rooted tree, 8

rotunda, 121

rotunda graph, 122

rotunda tree, 121

round flat, 58

round matroid, 58

satisfy, 22

saturated, 99

sentence, 22

simple graph, 7

simplicial decomposition, 65

simplicial ordering, 95

simplicial vertex, 64

source, 29

span, 10

strand, 54

string, 20

strongly chordal, 74

strongly chordal graph, 76

subgraph, 7

subtree, 8

supersolvable, 93

supersolvably saturated, 100

tree, 8

tree decomposition of a graph, 12

treewidth, 14

triangle, 74

unnecessary, 56

unrooted tree, 8

vertex cut, 63 
vertical, 10

weakly chordal, 73

weakly chordal graph, 76

width, 120

width of a branch decomposition of a

graph, 14

width of a branch decomposition of a

matroid, 17

word, 20 


\section{Bibliography}

[1] Anton, H. and Rorres, C. (2005) Elementary Linear Algebra: Application Version. John Wiley and Sons, Inc., Drexel University, ninth edition edn.

[2] Arnborg, S., Lagergren, J., and Seese, D. (1991) Easy problems for treedecomposable graphs. Journal of Algorithms, 12, 308-340.

[3] Barahona, F. and Grotschel, M. (1986) On the cycle polytope of a binary matroid. Journal of Combinatorial Theory B, 40, 40 - 62 .

[4] Bevern, R. v., Downey, R. G., Fellows, M. R., Gaspers, S., and Rosamond, F. A. (2013) Myhill-Nerode methods for hypergraphs. International Symposium on Algorithms and Computation: Algorithms and Computation, pp. 372-382.

[5] Bodlaender, H. L. (1993) A tourist guide through treewidth. Acta Cybernetica, 11.

[6] Bodlaender, H. L. (1996) A linear-time algorithm for finding treedecompositions of small treewidth. SIAM J. Comput., 25, 1305-1317.

[7] Bodlaender, H. L., Heggernes, P., and Telle, J. A. (2015) Recognizability equals definability for graphs of bounded treewidth and bounded chordality. Proceedings EUROCOMB 2015: Electronic Notes in Discrete Mathematics, pp. 1-5.

[8] Bojanczyk, M. and Pilipczuk, M. (2016) Definability equals recognizability for graphs of bounded treewidth. Proceedings of the 31st Annual ACM/IEEE Symposium on Logic in Computer Science, pp. 407-416. 
[9] Bondy, A. and Murty, M. R. (2008) Graph Theory. Graduate Texts in Mathematics, Springer.

[10] Bonin, J. and deMier, A. (2004) T-uniqueness of some families of kchordal matroids. Advances in Applied Mathematics, 32, 10 - 30.

[11] Brandstadt, A., Le, V. B., and Sprinrad, J. (1999) Graph Classes: A Survey. SIAM Monographs on Discrete Mathematics and Applications.

[12] Brylawski, T. (1975) Modular constructions for combinatorial geometries. Transactions of the American Mathematical Society, 203.

[13] Buchi, J. R. (1960) Weak second-order arithmetic and finite automata. Mathematical Logic Quarterly, 6, 66-92.

[14] Cordovil, R., Forge, D., and Klein, S. (2004) How is a chordal graph like a supersolvable binary matroid? Discrete Mathematics, 288, 164-172.

[15] Courcelle, B. (1990) The monadic second-order of graphs. i. recognizable sets of finite graphs. Information and Computation, 85, 63.

[16] Courcelle, B. (1991) The monadic second-order logic of graphs v: on closing the gap between definability and recognizability. Theoretical Computer Science, 80, 153-202.

[17] Dinneen, M. J. (1997) Practical enumeration methods for graphs of bounded pathwidth and treewidth. Centre for Discrete Mathematics and Theoretical Computer Science, CDMCTCS-055.

[18] Dirac, G. A. (1961) On rigid circuit graphs. Abhandlungen aus dem Mathematischen Seminar der Universitt Hamburg, 25, 71 - 76.

[19] Doner, J. (1970) Tree acceptors and some of their applications. Journal of Computing System Science, 4, 45.

[20] Downey, R. G. and Fellows, M. R. (2013) Fundamentals of Parameterized Complexity. Springer-Verlag, London.

[21] Galinier, P., Habib, M., and Paul, C. (1995) Chordal graphs and their clique graphs. WG 1995: Graph Theoretic Concepts in Computer Science, pp. 358-371. 
[22] Geelen, J., Gerards, B., and Whittle, G. (2006) On Rota's conjecture and excluded minors containing large projective geometries. Journal of Combinatorial Theory, Series B, 405-425.

[23] Habib, M. and Stacho, J. (2012) Reduced clique graphs of chordal graphs. European Journal of Combinatorics, 33, 712-735.

[24] Hayward, R. B. (1985) Weakly triangulated graphs. Journal of Combinatorial Theory, B, 200-208.

[25] Hliněný, P. (2006) Branch-width, parse trees, and monadic second-order logic for matroids. J. Comb. Theory Ser. B, 96, 325-351.

[26] Hliněný, P., Oum, S.-I., Seese, D., and Gottlob, G. (2008) Width parameters beyond tree-width and their applications. Comput. J., 51, 326-362.

[27] Hliněný, P. and Whittle, G. (2006) Matroid tree-width. European Journal of Combinatorics, 27, 1117-1128.

[28] Jaffke, L. and Bodlaender, H. L. (2015) Msol-definability equals recognizability for Halin graphs and bounded degree k-outerplanar graphs. arXiv:1503.01604 [cs.LO].

[29] Kabanets, V. (1996) Recognizability equals definability for partial kpaths. Masters thesis, Simon Fraser University.

[30] Kabanets, V. (1997) Recognizability equals definability for partial kpaths. ICALP'g'7 LNCS, 1256, 805-815.

[31] Kaller, D. (1997), Definability equals recognizability of partial 3-trees.

[32] Koutsonas, A., Thilikos, D., and Yamazaki, K. (2014) Outerplanar obstructions for matroid pathwidth. Discrete Mathematics, 315-316, 95101.

[33] Koutsonas, A., Thilikos, D. M., and Yamazaki, K. (2014) Outerplanar obstructions for matroid pathwidth. Discrete Math., 315-316, 95-101.

[34] Kruskal, J. B. (1956) On the shortest spanning subtree of a graph and the traveling salesman problem. Proceedings of the American Mathematical Society, 7, 48-50. 
[35] Kung, J. P. S. (1986) Numerically regular hereditary classes of combinatorial geometries. Geom. Dedicata, 21, no. 1, 85-110.

[36] Lapoire, D. (1998) Recognizability equals monadic second-order definability for sets of graphs of bounded tree-width. STACS'98 LNCS, pp. 618-628.

[37] Lidl, R. and Niederreiter, H. (1994) Introduction to finite fields and their applications. Cambridge University Press, revised edn.

[38] Mayhew, D., (2018) private conversation.

[39] Mayhew, D., Newman, M., and Whittle, G. (2014) Is the missing axiom of matroid theory lost forever? The Quarterly Journal of Mathematics, 65, 1397-1415.

[40] McCarthy, P. (1966) Algebraic Extensions of Fields. Blaisdell Publishing Company.

[41] Oxley, J. (2006) Matroid Theory. Oxford University Press.

[42] Robertson, N. and Seymour, P. (1983) Graph minors. I. excluding a forest. Journal of Combinatorial Theory B, 33, 39-61.

[43] Robertson, N. and Seymour, P. D. (1991) Graph minors. X. obstructions to tree-decomposition. Journal of Combinatorial Theory, Series B, 52, 153-190.

[44] Seese, P. H. D. (2004) On Decidability of MSO Theories of Representable Matroids, vol. 3162, book section 9, pp. 96-107. Springer, Berlin, Heidelberg.

[45] Seymour, P. and Thomas, R. (1994) Call routing and the rat catcher. Combinatorica, 14, 217-241.

[46] Sipser, M. (2012) Introduction to the Theory of Computation. Cengage Learning.

[47] Stanley, R. P. (1972) Supersolvable lattices. Algebra Universalis, 2, 197 $-217$. 
[48] Whittle, G. P. (1985) Some Aspects of the Critical Problem for Matroids. $\mathrm{PhD}$ thesis, University of Tasmania.

[49] Ziegler, G. M. (1991) Binary supersolvable matroids and modular constructions. Proceedings of the American Mathematical Society, 113, 817 $-829$. 